\title{
OUT-OF-LOOP COMPENSATION METHOD FOR OP-AMPS DRIVING HEAVY CAPACITIVE LOADS
}

\author{
A Thesis \\ presented to \\ the Faculty of California Polytechnic University, \\ San Luis Obispo \\ In Partial Fulfillment \\ of the Requirements for the Degree \\ Master of Science in Electrical Engineering
}

by

Shubham Gandhi

March 2016 
(C) 2016

Shubham Gandhi

ALL RIGHTS RESERVED 
TITLE:

AUTHOR:

DATE SUBMITTED:

COMMITTEE CHAIR:

COMMITTEE MEMBER:

COMMITTEE MEMBER:
Out-of-Loop Compensation Method for Opamps Driving Heavy Capacitive Loads

Shubham Gandhi

March 2016

Vladimir Prodanov, Ph.D.

Associate Professor of Electrical Engineering

Tina Smilkstein, Ph.D.

Associate Professor of Electrical Engineering

Xiao-Hua (Helen) Yu, Ph.D.

Professor of Electrical Engineering 


\section{ABSTRACT \\ Out-of-Loop Compensation Method for Op-amps Driving Heavy Capacitive Loads Shubham Gandhi}

It is well known that real op-amps do not share most of the desirable characteristics of an ideal one, particularly those of gain and output impedance. When presented with a capacitive load, such as a MOSFET or ADC, feedback in an op-amp circuit can quickly become unstable. This thesis studies and characterizes an op-amp's output impedance and how its interaction with this type of load creates a parasitic pole which leads to instability. Applying ideas from feedback control theory, a model for studying the problem is developed from which a generalized method for compensating the undesirable circumstance is formulated.

Even in a zero-input state, many real op-amps driving capacitive loads can experience unforced oscillations. A case study is performed with three commonly used devices. First, the output impedance is determined by its dependence on the unity-gain bandwidth, load capacitance, and oscillation frequency. It is fitted into a second-order feedback control model that allows for an analytical study of the problem. It is then shown that a carefully designed passive network can be introduced between the load and op-amp to obtain a properly damped system free of oscillation and well-behaved.

Using a shunt resistor is a known and commonly used method for lowering an opamp's output impedance to gain stability. This work considers the converse addition of a series capacitor to instead lower the load capacitance seen by the op-amp, a seemingly complementary method that achieves the same goal. A generalized, composite compensation method is developed that uses both the shunt resistor and series capacitora strategy not yet found in literature. Relevant formulas for damping ratio and natural frequency are derived that allow the design of a passive compensation network. Furthermore, tradeoffs between compensation, voltage swing, current consumption, and power usage are considered.

An emphasis is placed on comparing simulated versus real circuits to highlight the fact that any problem is much worse in real-life than in a simulation. SPICE models and programs aim to de-idealize certain device characteristics, but often cannot account for environmental conditions and manufacturing variance. Thus, an importance is placed on experimental verification guided by simulations.

Keywords: op-amp, capacitive load, output impedance, stability, feedback, compensation, passive network, SPICE 


\section{ACKNOWLEDGMENTS}

I would like to express my deepest gratitude to Dr. Vladimir Prodanov for all the invaluable advice and insightful conversations during the development of the ideas in this thesis. Its completion would not have been possible without his guidance. I am also grateful to my parents and brother for their support and encouragement. 


\section{TABLE OF CONTENTS}

Page

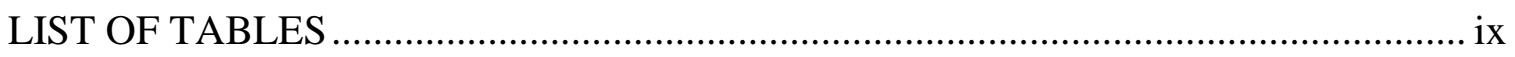

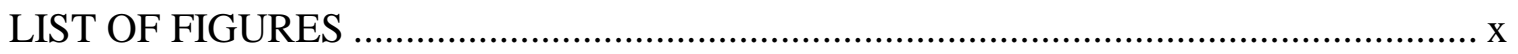

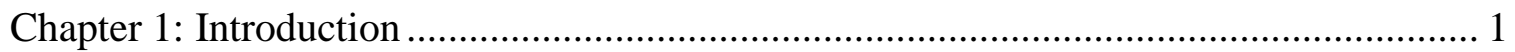

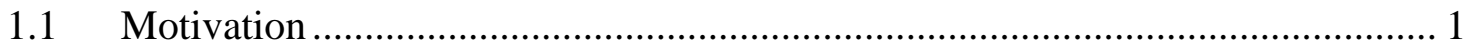

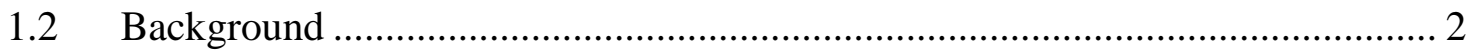

1.3 Where Output Impedance Comes From...................................................... 4

1.4 Problem Statement and Procedure Summary ............................................. 5

Chapter 2: Characterization Methods and Case Study …............................................ 7

2.1 Method for Characterizing Open-Loop Gain and GBW ................................ 7

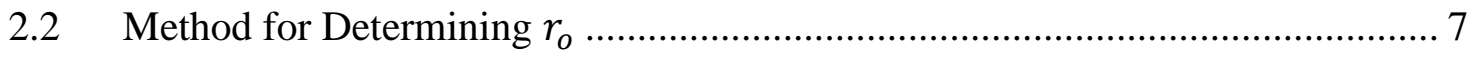

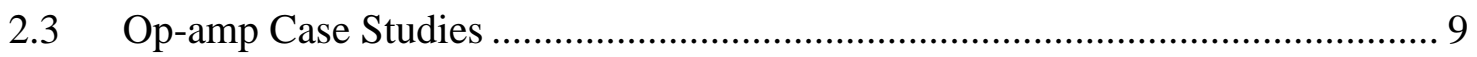

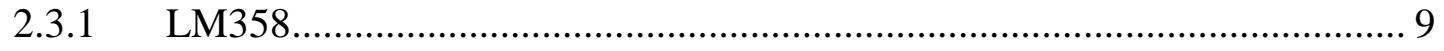

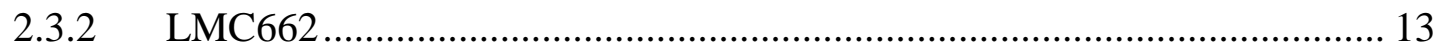

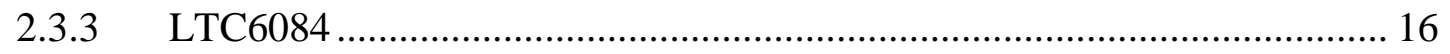

2.4 Summarized Results and Discussion........................................................ 19

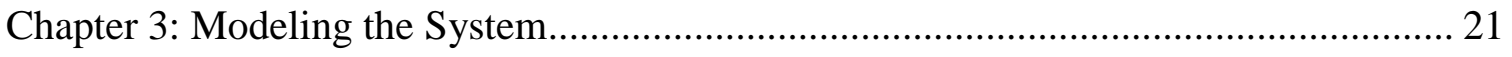

3.1 Review of Relevant Feedback Control Theory ......................................... 21

3.2 Summing Junction and Integrator Model of an Op-Amp .............................. 22

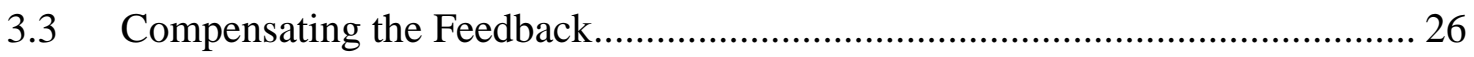

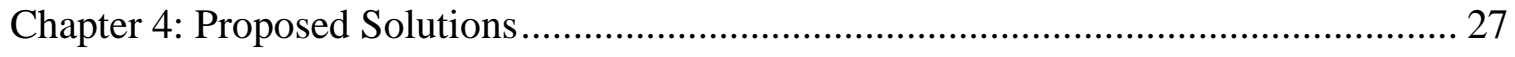

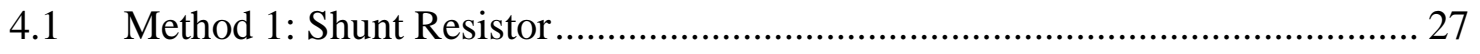

4.1.1 Closed-Loop Analysis with Example Application ................................. 27

4.1.2 Open-Loop Analysis with Example Application ................................... 29 
4.1.3 Practical Implementation Requires an Additional Capacitor. 30

4.2 Method 2: Series Capacitor ........................................................................ 32

4.2.1 Closed-Loop Analysis with Example Application ................................. 33

4.2.2 Open-Loop Analysis with Example Application .................................... 34

4.2.3 Practical Implementation Requires an Additional Resistor ...................... 35

4.3 Proposed Composite Method - Using Both ............................................ 37

4.3.1 Closed-Loop Analysis with Example Application .................................. 38

4.3.2 Open-Loop Analysis with Example Application .................................... 39

4.3.3 Practical Implementation Requires Two Components ............................ 41

Chapter 5: Practical Design of the Composite Compensation Network.......................... 43

5.1 Determine the Actual Device Characteristics ............................................ 43

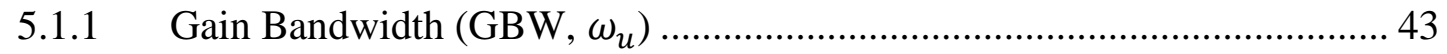

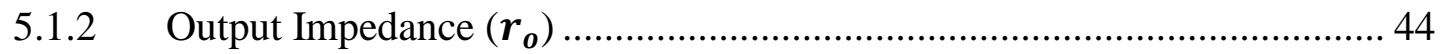

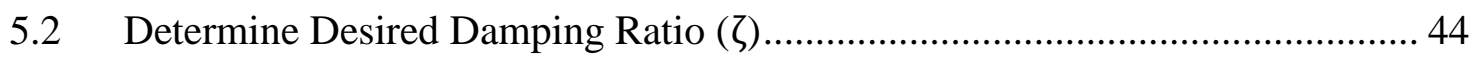

5.3 Consider Compensation Type for Application.......................................... 45

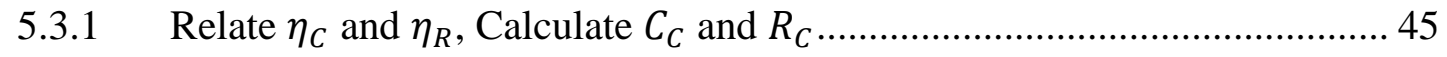

5.3.2 Determine Values for DC-Blocking $C_{1}$ and DC-Conducting $R_{1} \ldots \ldots \ldots \ldots . . . . .46$

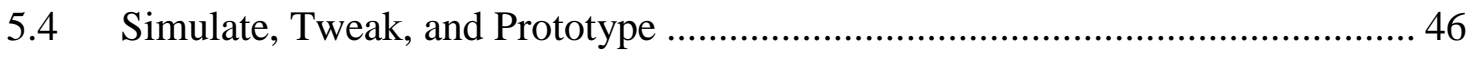

Chapter 6: Lab Experiment with Compensated Op-Amps ......................................... 47

6.1 LM358 Composite Compensation ..................................................... 47

6.2 LMC662 Composite Compensation ........................................................... 49

6.3 LTC6084 Composite Compensation ....................................................... 51

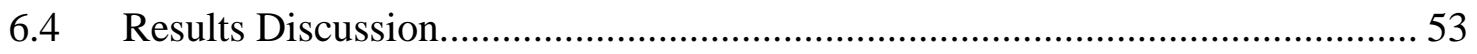

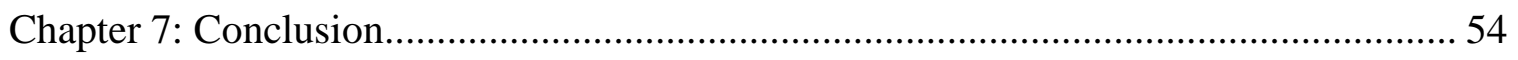

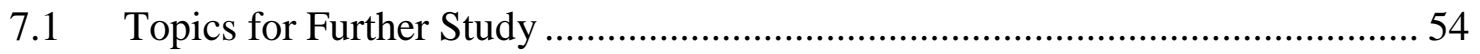


REFERENCES

\section{APPENDICES}

A1 Deriving the Idealized Op-Amp Model ........................................................ 56 


\section{LIST OF TABLES}

Table

Table 1. Simulation data of a voltage follower with the LM358 to determine its

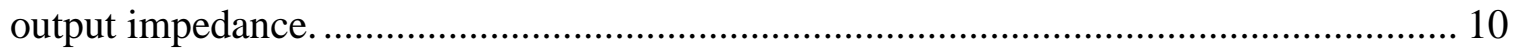

Table 2 . The secondary pole is displaced to an extremely low frequency.

Table 3. Simulation data of a voltage follower with the LMC662 to determine its

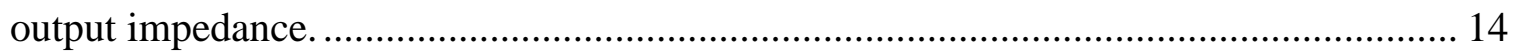

Table 4. The secondary pole is displaced to an extremely low frequency. 16

Table 5. Simulation data of a voltage follower with the LTC6084 to determine its

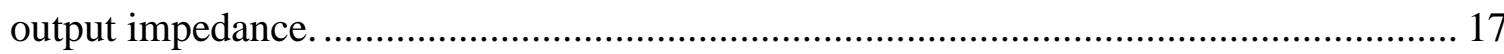

Table 6 . The secondary pole is displaced to an extremely low frequency. 19

Table 7. Expected vs. mesaured values of GBW and output imepdance (for 1 and

$10 \mathrm{nF}$ loads only). 19

Table 8. Compensation components calculated for a critically damped response.

Table 9. Resistors and capacitors rounded to standard values so damping ratio stays

above 1 .

Table 10. Compensation components calculated for a critically damped response.

Table 11. Resistors and capacitors rounded to standard values so damping ratio stays

above 1 .

Table 12. Compensation components calculated for a critically damped response.

Table 13. Resistors and capacitors rounded to standard values so damping ratio stays

above 1 


\section{LIST OF FIGURES}

Figure $\quad$ Page

Figure 1. Real op-amps have finite gain and non-zero output impedance........................ 1

Figure 2. Attempting to drive a large enough capacitance leads to instability. .................. 2

Figure 3. Secondary pole beyond the unity-gain frequency gets pulled in...................... 3

Figure 4. Secondary pole becomes parasitic because it reduces phase margin. ................ 4

Figure 5. Output impedance is determined by the bias currnet of the output stage........... 5

Figure 6. Vn is non-zero in a real circuit, and $\mathrm{Vo} / \mathrm{Vn}$ can be used to determine open

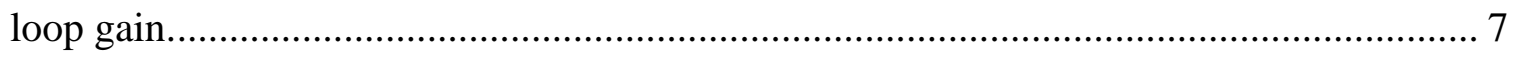

Figure 7. Oscillating Vout can be used to determine output impedance. ......................... 8

Figure 8. Shifted secondary pole adds another $20 \mathrm{~dB} / \mathrm{dec}$ decrease that also shifts the

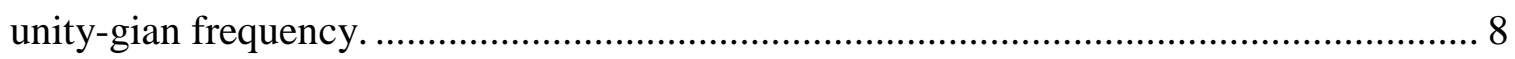

Figure 9. Gain peaks at the loop's natural frequency, at which the ringing oscillates. .... 10 Figure 10. LM358 powered from $\pm 5 \mathrm{~V}$ supply configured as follower with grounded input (above). $\mathrm{V}_{\text {out }}$ is probed with loads $4.7 \mathrm{nF}$ (top left) and $47 \mathrm{nF}$ (bottom left).......... 11

Figure 11. Open loop gain drops at 20db/dec as expected. Average GBW is $1.18 \mathrm{MHz} .12$ Figure 12. Oscillation frequency and output impedance is much higher than predicted by simulations.

Figure 13. Gain peaks at the loop's natural frequency, at which the ringing oscillates. 13

Figure 14. LMC662 powered from $\pm 5 \mathrm{~V}$ supply configured as follower with grounded input (above). $\mathrm{V}_{\text {out }}$ is probed with loads $4.7 \mathrm{nF}$ (top left) and $47 \mathrm{nF}$ (bottom left). 14

Figure 15. Open loop gain drops at 20db/dec as expected. Average GBW is $1.79 \mathrm{MHz} .15$ Figure 16. Oscillation frequency and output impedance is much higher than predicted by simulations. 15

Figure 17. Gain peaks at the loop's natural frequency, at which the ringing oscillates. .. 16 Figure 18. LTC6084 powered from $\pm 2.5 \mathrm{~V}$ supply configured as follower with grounded input (above). $\mathrm{V}_{\text {out }}$ is probed with loads $4.7 \mathrm{nF}$ (top left) and $47 \mathrm{nF}$ (bottom left). 17 
Figure 19. Open loop gain drops at $20 \mathrm{db} / \mathrm{dec}$ as expected. Average GBW is $2.1 \mathrm{MHz} . .18$ Figure 20. Oscillation frequency and output impedance is much higher than predicted by simulations. 18

Figure 21. Generic controls system with $\mathrm{A}(\mathrm{s})$ forwardforward and $\mathrm{F}(\mathrm{s})$ feedback. ........ 21

Figure 22. Op-amp modeled as smming junction, integrator, and output impedance...... 23

Figure 23. Ouput impedance and load are lumped into a transfer block....................... 23

Figure 24. Poles become more complex as damping ratio decreases. ........................... 25

Figure 25. Root locus of op-amp modeled as a control system. Dominant and secdonary poles come together and become complex. 25

Figure 26. T(s) can be supplemented with a compensation network that could stabalize feedback. 26

Figure 27. A resitor shunted to ground at the op-amp output biases the output

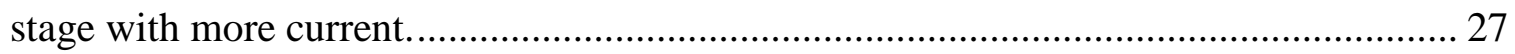

Figure 28. Secondary pole is shifted out, but open-loop gain is reduced. 29

Figure 29. R1 is typically small in value, so $\mathrm{C} 1$ prevents it from conducting a large current. 30

Figure 30. Shunt compensation eliminates ringing and gain peaking. 31

Figure 31. At lower frequencies, $\mathrm{C} 1$ conducts 20 times more current than the load........ 32 Figure 32. A series capacitor reduces the load capacitance seen. The "I|" operator

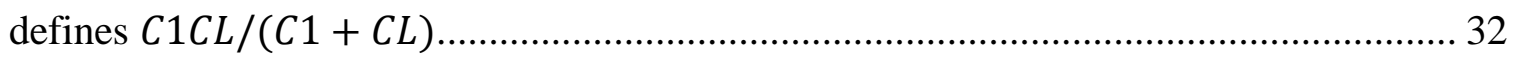

Figure 33. Secondary pole is shifted out, but open-loop gain is reduced....................... 34

Figure 34. The output node becomes isolated, so an additional R1 is needed to

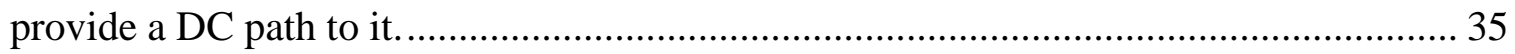

Figure 35. Series compensation eliminates ringing and gain peaking .......................... 36

Figure 36. At higher frequencies, the op-amp needs a larger output swing to maintain

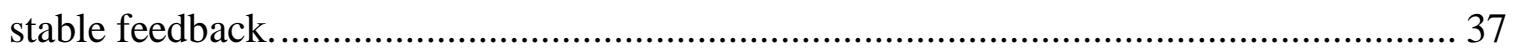

Figure 37. Using shunt and series comepnsation, outptut impedance and load

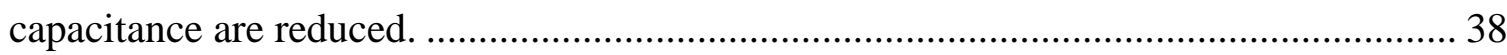

Figure 38. Secondary pole is shifted out, but open-loop gain is reduced. ....................... 40 Figure 39. Additional DC blocking capacitor and DC conducting resistor are required.. 41 Figure 40. Composit compensation eliminates ringing and gain peaking. 
Figure 41. At higher frequencie, the op-amp must swing $4.35 \mathrm{~dB}$ more to maintain unity feedback.

Figure 42. Natural frequency is damped out when there is no input. About $15 \%$ overshoot is present with a $1 \mathrm{~V}$ impulse, and some ringing is also appears in the falling edge of the $1 \mathrm{nF}$ load. This is likely due to the non-symmetric design of the output stage. 48

Figure 43. $1 \mathrm{nF}$ is driven without any noticable distrotion. For the larger load, $4.7 \mathrm{nF}$, some evidence of distortion is apparent because of output stage deadzones creeping in along the peaks and troughs.

Figure 44. Unfortunately, LMC662 is a bit more stubborn with its natural frequency, a greater damping ratio is required. The falling edge on a $1 \mathrm{nF}$ load is well-behaved, but the rising edge rings for about $15 \mu \mathrm{s}$ most likely due to a nonsymmetrical output impedance.

Figure 45. The compensated amplifier is able to drive capacitive loads with a

linear signal without any noticeable distortion or ringing artifacts.

Figure 46. $1 \mathrm{nF}$ and $4.7 \mathrm{nF}$ loads have about $50 \%$ and $20 \%$ overshoot, respectively, that die out very quickly within about $5 \mu$ s. Edge transitions are nearly symmetric, which underscores the op-amp's symmetric and linear design.

Figure 47. The compensated amplifier is able to drive capacitive loads with a linear signal without any noticeable distortion or ringing artifacts.

Figure 48. Instability from driving a capacitive load with grounded input (zero-input response)

Figure 49. Standard two-pole model of an op-amp with gain DC gain block and unity feedback. 56

Figure 50. Two-pole model can be simplifed to an idealized type-I second order model 


\section{Chapter 1: Introduction}

\subsection{Motivation}

Operational amplifiers have come a long way since Robert Widlar's original design of the $\mu$ A702 in the 1960's, the Pleistocene of modern electronics. The competitive nature of design and iterative improvement has proliferated the availability of options, and has made finding the right part akin to finding a needle in a pile of needles. Although the origin story of op-amps has a remarkable retrospective appeal, this work is more concerned with the current state of affairs and a particular issue that many engineers are faced with when dealing with this type of integrated circuit (IC).

The ideal characteristics of an op-amp are well defined and understood. Infinite gain, infinite input impedance, zero output impedance indirectly imply infinite slew rate, zero input current, zero offset voltage, and infinite bandwidth h However, it is also well understood that real op-amps do not share many of these characteristics. Semiconductor fabrication processes refined over the decades have enabled input impedance to approach infinity (for practical purposes) through the use of FET inputs. Yet, the ideality of gain and output impedance for real devices has to be loosely defined by "good enough" depending on the application. With the IC already fabricated and possibly selected for use, the curious cases of "not good enough" that pose a problem are worth solving with proper analysis.

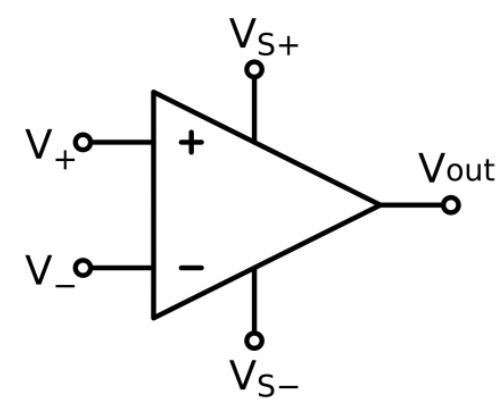

Ideal

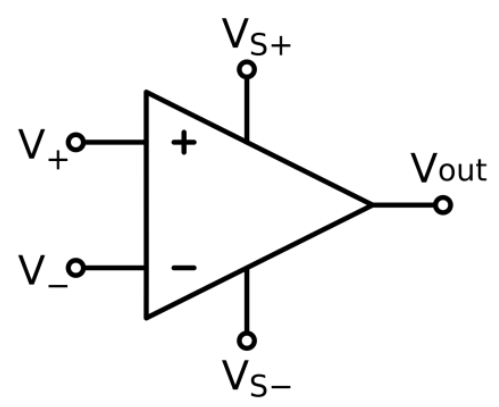

Real

$$
\operatorname{Av}=\infty, \operatorname{Rin}=\infty, \text { Rout }=0
$$

$$
\text { Av }<\infty, \text { Rin } \cong \infty, \text { Rout }>0
$$

Figure 1. Real op-amps have finite gain and non-zero output impedance. 
Most general purpose op-amps are carefully designed to drive largely resistive loads that may be minimally capacitive - 10's or 100's of $\mathrm{pF}$ at most. But what happens when the load is heavily capacitive? The type of load is usually not a matter of choice. Capacitance may not necessarily come from an actual capacitor, but may come from a device that is capacitive in constitution such as the gate of a FET, the length of a coaxial cable, or it may actually be the holding capacitor of an ADC. These can easily be well into the $\mathrm{nF}$ range.

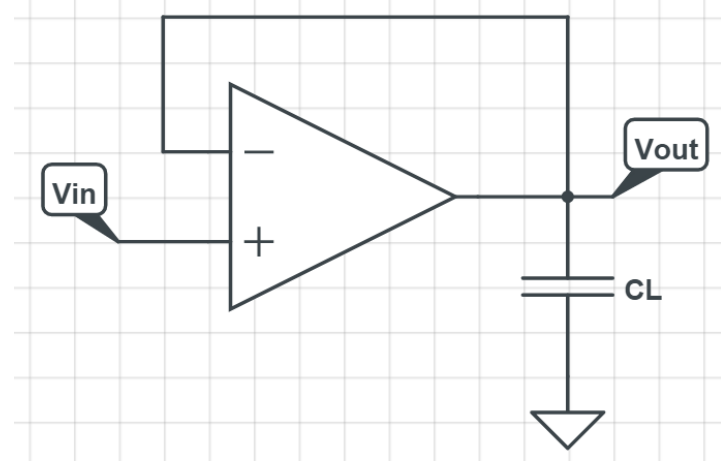

Figure 2. Attempting to drive a large enough capacitance leads to instability.

Unsurprisingly, this is a case when even the strongest of feedback will experience instability. The output impedance and load capacitance form a low-pass filter with a cutoff frequency that could be less than the unity-gain frequency $\left(f_{u}\right)$ of the op-amp, effectively adding a parasitic pole to the device's open-loop response. As a result, phase margin becomes eliminated turning the circuit into an oscillator. Dealing with this consequence is the main focus of this thesis.

\subsection{Background}

The open-loop response of most op-amps contains two main poles: the dominant and the secondary. Gain begins to roll off at $20 \mathrm{~dB} / \mathrm{dec}$ at the low frequency dominant pole, and another $20 \mathrm{~dB} / \mathrm{dec}$ is added at the high frequency secondary pole. 


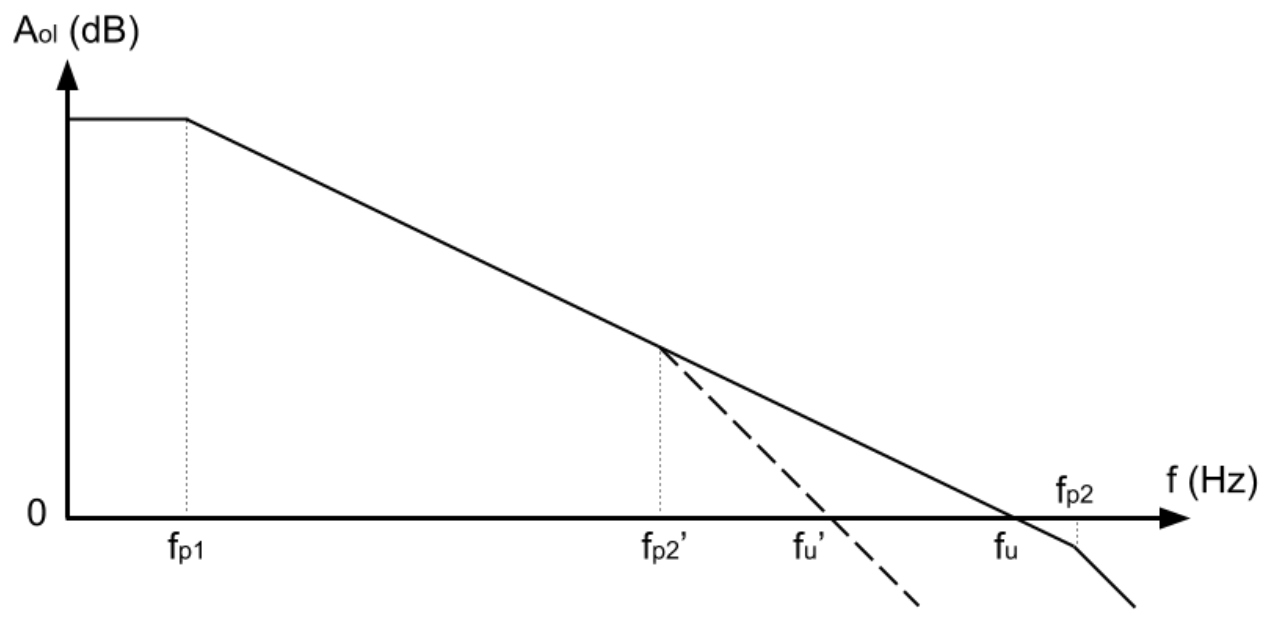

Figure 3. Secondary pole beyond the unity-gain frequency gets pulled in.

By design, the secondary pole on most op-amps is carefully placed beyond the device's unity-gain frequency. As a result, it introduces minimal phase shift so a large phase margin can be maintained. The portion of the open-loop response beyond the unit-gain frequency is typically omitted in datasheets as it becomes irrelevant while the amplifier operated within the load capacitance rating (typically 10's to 100's of $\mathrm{pF}$ ). Thus, it is important to examine what happens in the unavoidable situations when operation is out of spec (loads in the $\mathrm{nF}$ range).

It turns out that the location of the secondary pole depends on the capacitance present on the amplifier's output node. A "heavy" load has the effect of pulling this pole inwards such that it becomes parasitic to the open-loop response. This occurs because the output impedance and load capacitance form a low-pass filter well below the unity-gain frequency. Examining the magnitude and phase response of a generic amplifier that is capacitively loaded, it becomes clear there is cause for concern, as illustrated by Figure 4 . 


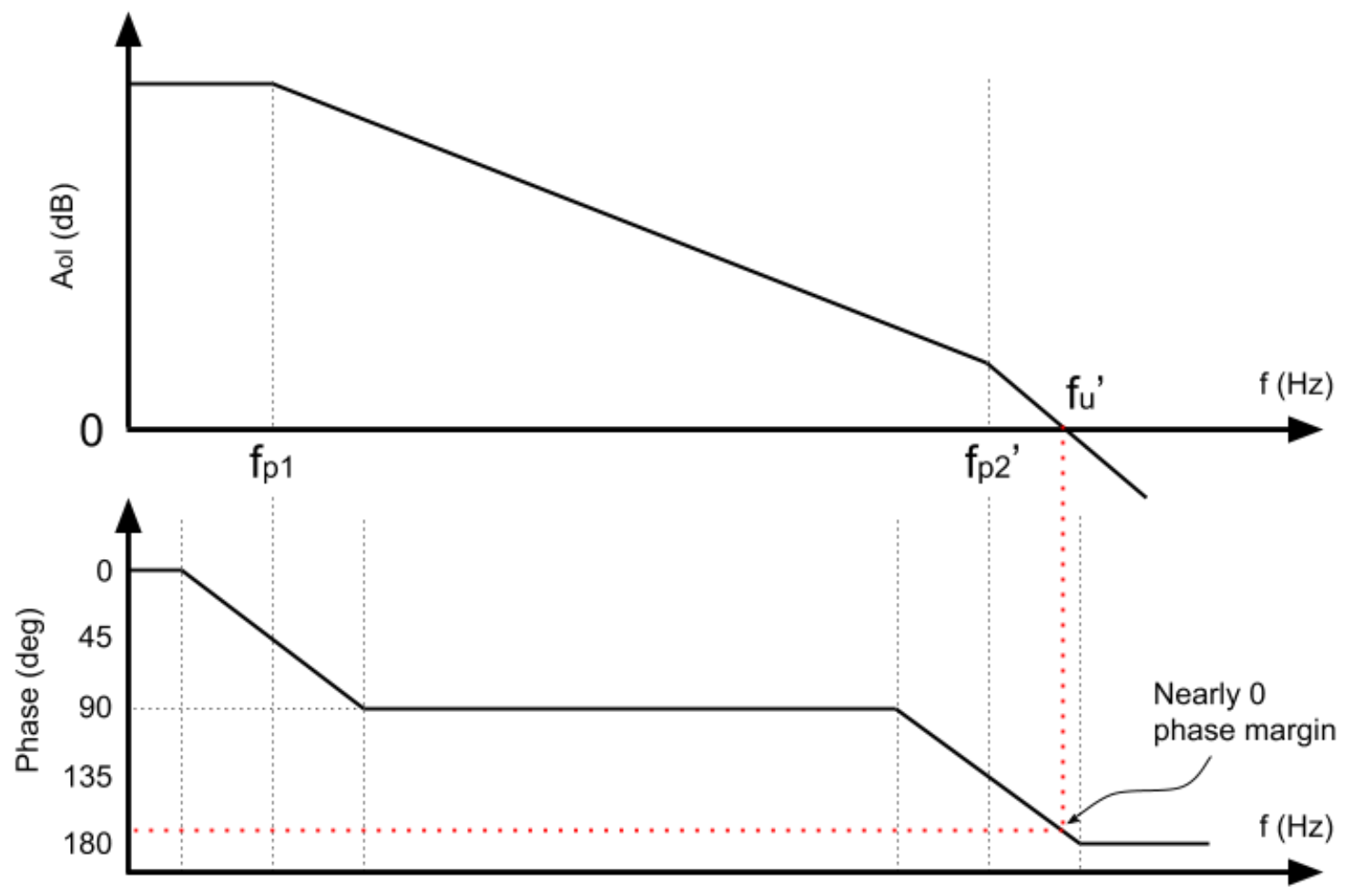

Figure 4. Secondary pole becomes parasitic because it reduces phase margin.

Op-amps are typically designed to maintain a phase margin of greater than $60^{\circ}$ when loaded at the maximum rating. With heavier loading, the new parasitic pole contributes enough phase shift that the amplifier has a gain greater than $0 \mathrm{~dB}$ when the input signal has shifted by almost $180^{\circ}$ (inverted) at the output. If this output signal is fed back into the non-inverting input, negative feedback actually becomes positive feedback which causes the circuit to oscillate even when the input is zero. This is known as the Nyqist Staibilty Criterion.

\subsection{Where Output Impedance Comes From}

To fully grasp the extent of the problem, it may be prudent to examine where the output impedance actually comes from. Figure 5 shows the transistor level schematic of the popular LM358 op-amp with the output stage outlined in red. Assuming $\mathrm{R}_{\mathrm{SC}}$ to be small in value, the output node looks into a pair of BJT emitter terminals. 


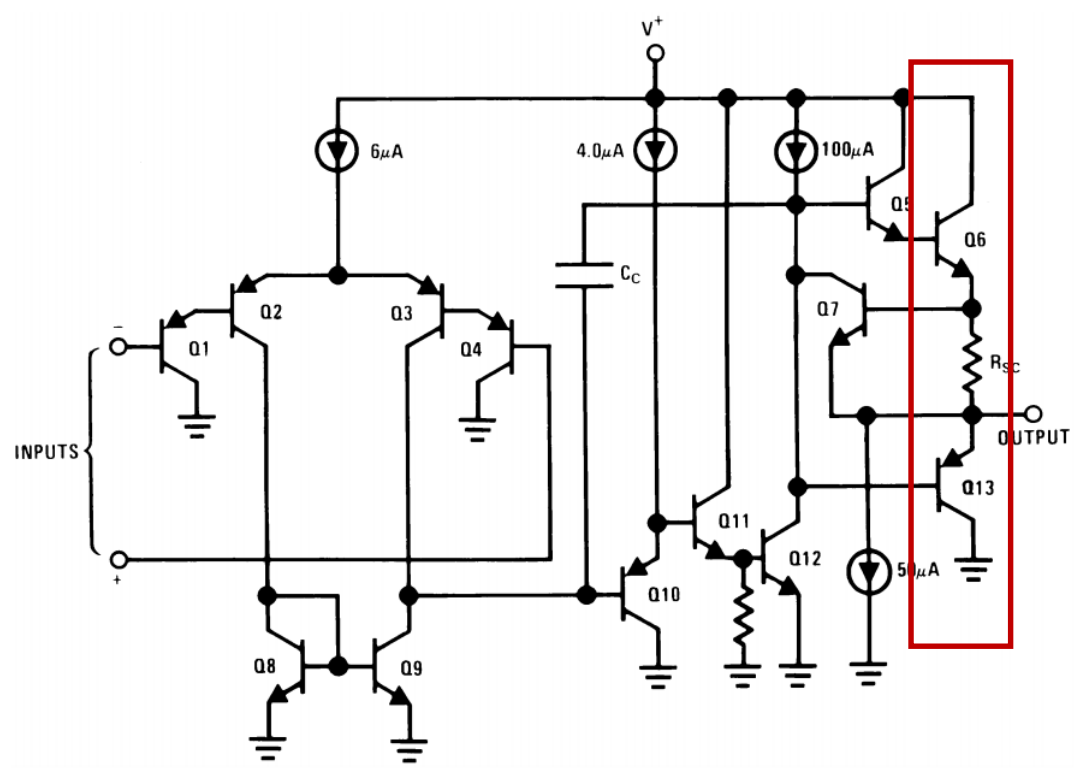

Figure 5. Output impedance is determined by the bias currnet of the output stage.

Recall that, from the small signal model of a BJT, the resistance looking the emitter is $1 / g_{m}$, where $g_{m}$ is the transconductance of the transistor set by the bias current. This would imply that the output impedance, $r_{o}$, of the op-amp is a small signal measure that is relatively small in magnitude. It is this intangible quantity that ends up creating a pole with the load capacitance to compromise feedback stability.

It should be noted that although $r_{o}$ has a reactive component, it is largely resistive. For the purposes of this study, it is referred to as impedance, but is taken to be only resistive.

\subsection{Problem Statement and Procedure Summary}

So far it has been established that capacitive loading displaces an op-amp's secondary pole in a turn for the worse. As phase margin regresses toward $0^{\circ}$ feedback begins to wobble, and the system produces undesirable oscillations. Even with no input (i.e. tied to ground), the output can have a non-zero, periodic steady state. The rest of this study takes on an analytical treatment of this problem that is concluded by experimentally verifying a generalized solution.

Fist, a method for measuring open-loop response and gain-bandwidth (GBW) product of a real device are discussed followed by a method for determining the output 
impedance, $r_{o}$. Next, a case study presents lab experiment data for three commonly used op-amps where impulse response and oscillation frequency due to instability are captured. Using this frequency and a formula derived in a later chapter $r_{o}$ is estimated. For any experiments performed, actual results are also compared with simulated results and any disparity is discussed.

Furthermore, a feedback model is developed by taking the op-amp to be a summing junction, integrator, and a resistor (output impedance) in a control loop loaded by a capacitor. After deriving equations for the natural frequency and damping ratio, it is shown that a passive compensation network inserted between the load and output impedance can be designed to critically dampen the instability. A general compensation strategy is then formulated for any given op-amp and load.

Finally, a lab experiment is performed with the same three op-amps which are compensated to successfully drive capacitive loads ranging from $1 \mathrm{nF}$ to $100 \mathrm{nF}$. 


\section{Chapter 2: Characterization Methods and Case Study}

\subsection{Method for Characterizing Open-Loop Gain and GBW}

Figure 6 illustrates the test circuit used to do determine the open-loop response and GBW of a real device. Weak feedback is applied with large resistors configured to deliver a closed-loop gain of $|10|$, and a small-signal input sinusoid stimulates the system.

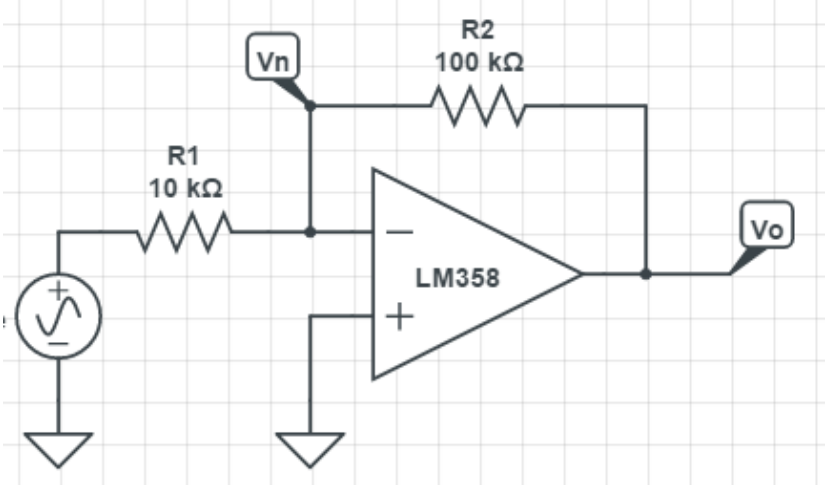

Figure 6. Vn is non-zero in a real circuit, and $\mathrm{Vo} / \mathrm{Vn}$ can be used to determine open loop gain.

Ideally, the inverting and non-inverting terminals should both be at the same potential, or virtually shorted; however this is not true of a real device. A commonly used characterization technique to determine open-loop gain at the input frequency is measure the ratio of the output magnitude to that of the inverting terminal (for this configuration)

Measuring this ratio while sweeping the input frequency, a graph of gain versus frequency can be generated. Furthermore, as the expression suggests, gain-bandwidth (GBW) product can be calculated by the product of gain and the frequency at which it is measured.

$$
A_{o l}=\frac{V o}{V n} \quad \Rightarrow \quad G B W=f_{i n} * A_{o l}
$$

\subsection{Method for Determining $\boldsymbol{r}_{o}$}

It is of greater importance that op-amp output impedance is treated as a smallsignal value and is properly characterized. The elusive nature of this quantity often makes it difficult to pin down because it depends on things such as operating frequency and 
output stage bias current. Though, to obtain a reasonable approximation, a very simple circuit is required: an op-amp configured as a voltage follower with a load capacitor.

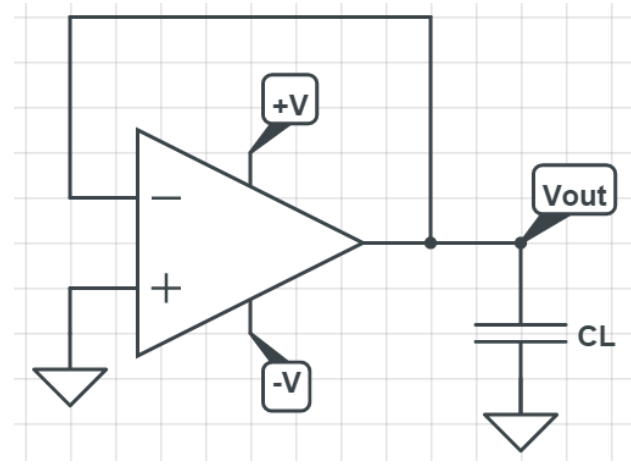

Figure 7. Oscillating Vout can be used to determine output impedance.

Feedback instability can be observed at Vout, which will oscillate at a frequency within a very narrow bandwidth dictated by the output impedance and load capacitance. As simulations will show in the following sections, there is a sharp, resonance-like peaking in the $\mathrm{AC}$ response of this circuit.

Examining in more detail the open-loop response with a parasitic pole, an interesting relationship can be derived between $r_{o}$ and other measurable quantities.

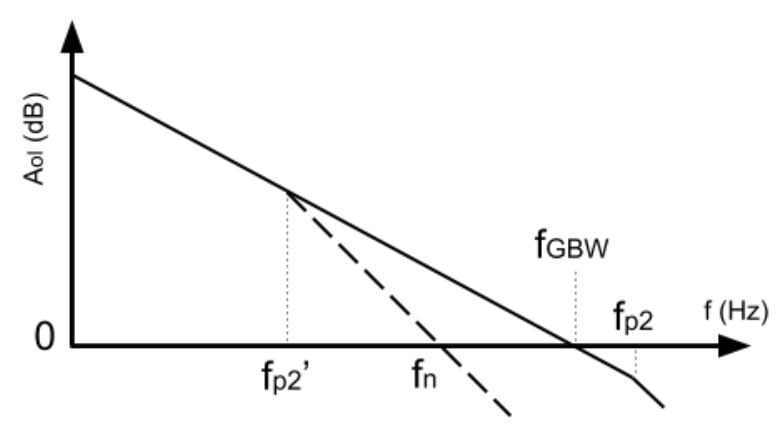

Figure 8 . Shifted secondary pole adds another $20 \mathrm{~dB} / \mathrm{dec}$ decrease that also shifts the unity-gian frequency.

Figure 8 illustrates the consequence of the secondary pole, $f_{p 2}$, being displaced to $f_{p 2}^{\prime}$, Here, $f_{G B W}$, the GBW frequency from the method in $\$ 2.1$, gets relocated to a new unitygain frequency denoted by $f_{n}$, which is the geometric mean of $f_{G B W}$ and $f_{p 2}^{\prime}$. 


$$
f_{n}=\sqrt{f_{G B W} * f_{p 2}^{\prime}}
$$

As previously discussed, the parasitic pole results from the low pass filter formed by $r_{o}$ and $C_{L}$, which would imply $f_{p 2}^{\prime}=\frac{1}{2 \pi * r_{o} * C_{L}}$. Furthermore, it may be a reasonable suspicion that the output will oscillate at approximately $f_{n}$ since phase shift is highest here $\left(f_{n}=f_{\text {osc }}\right)$. Fitting these two pieces of the puzzle into the equation above and rearranging terms, the following formula can be used to determine the value of $r_{o}$ that cooperates with a known $C_{L}$ to become parasitic.

$$
r_{o}=\frac{f_{G B W}}{2 \pi * C_{L} * f_{O S C}^{2}}
$$

It was also previously hinted that $f_{\text {osc }}$ has double significance as simulations will show resonance at this frequency as well, although real device measurements are likely to be dissimilar to simulated predictions.

\subsection{Op-amp Case Studies}

This section presents case studies for three op-amps that are popular among product designers and developers: LM358, LMC662, and [another] attempting to drive capacitive loads. Loads are varied from $1 \mathrm{nF}$ to $100 \mathrm{nF}$ and instability is viewed using simulations and oscilloscope captures. Then, the procedures from $\S 2.1$ and $\S 2.2$ are used to characterize the open-loop response, GBW, and $r_{o}$ of the real devices. Actual measurements are also compared against predictions from simulation and what is claimed in datasheets.

\subsubsection{LM358}

The popular LMx58 product line is only surpassed by the $\mu \mathrm{A} 741$ in ubiquity given its extremely low cost paired with mediocre characteristic by modern standards. As a general purpose op-amp, its works well enough for most applications of capacitive loads up to around $100 \mathrm{pF}$. Any load above this rating can cause feedback to become unstable. 


\section{Investigating Stability}

Simulations below present the op-amp's frequency and step responses to large loads under unity feedback.
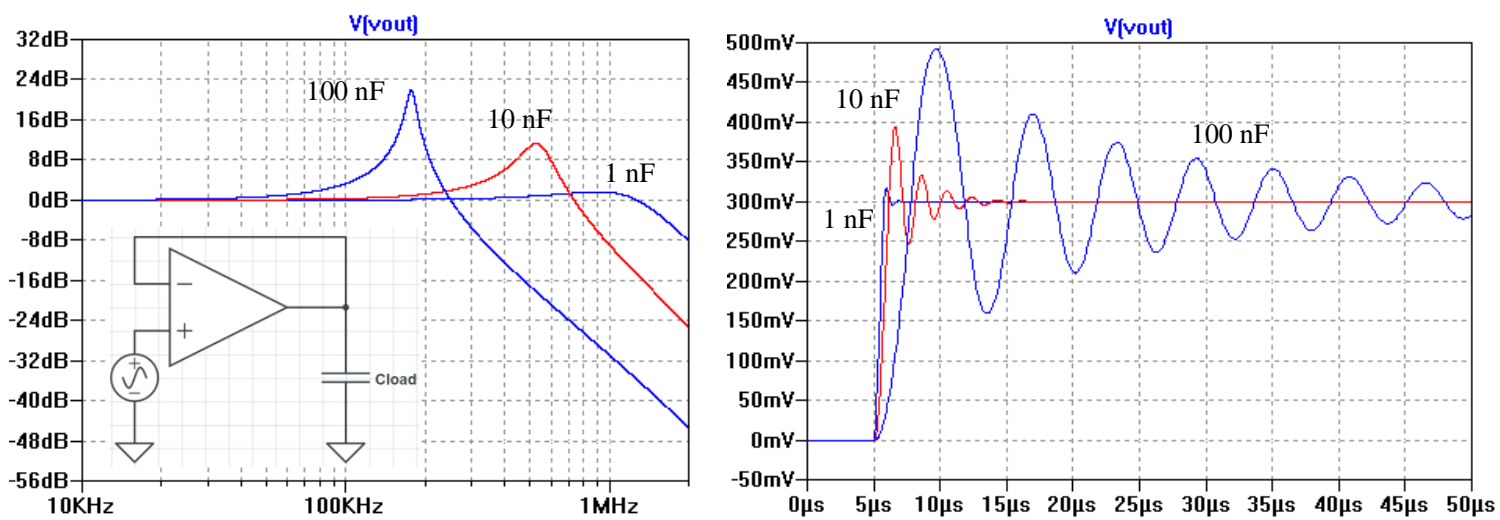

Figure 9. Gain peaks at the loop's natural frequency, at which the ringing oscillates.

Looking over three decades of heavy loading from $1 \mathrm{nF}$ to $100 \mathrm{nF}$, an increasing magnitude of instability ranges from a slight overshoot to severe ringing. Resonance-like gain peaking in the frequency domain manifests itself as ringing in time domain. By noting the gain peak location the parasitic pole and $r_{o}$ can be calculated using Equations 2.2 and 2.3 , the results of which are tabulated in Table 1 . GBW is assumed to $1.0 \mathrm{MHz}$ as claimed by datasheet.

\begin{tabular}{|r|r|r|r|}
\hline \multicolumn{1}{|c|}{ C,load (nF) } & f,peak (kHz) & f,pole $(\mathbf{k H z})$ & \multicolumn{1}{c|}{$\boldsymbol{r}_{\boldsymbol{o}}(\mathbf{\Omega})$} \\
\hline 1 & 910.4 & 828.8 & 192.0 \\
\hline 2.2 & 880 & 774.4 & 93.4 \\
\hline 4.7 & 713.5 & 509.1 & 66.5 \\
\hline 10 & 528 & 278.8 & 57.1 \\
\hline 22 & 369 & 136.2 & 53.1 \\
\hline 47 & 256.6 & 65.8 & 51.4 \\
\hline 100 & 177 & 31.3 & 50.8 \\
\hline
\end{tabular}

Table 1. Simulation data of a voltage follower with the LM358 to determine its output impedance.

As the load is made heavier, the geometric gap between $f_{\text {peak }}$ and $f_{\text {pole }}$ gets broader while $r_{o}$ settles toward an idealized value of $50 \Omega$.

It may sound reasonable to look at Figure 9 and judge the overshoot from a $1 \mathrm{nF}$ load as acceptable, but would be a misguided approach to solely rely on simulation data. 
Performing this experiment in a lab with an actual device tells a completely different story.
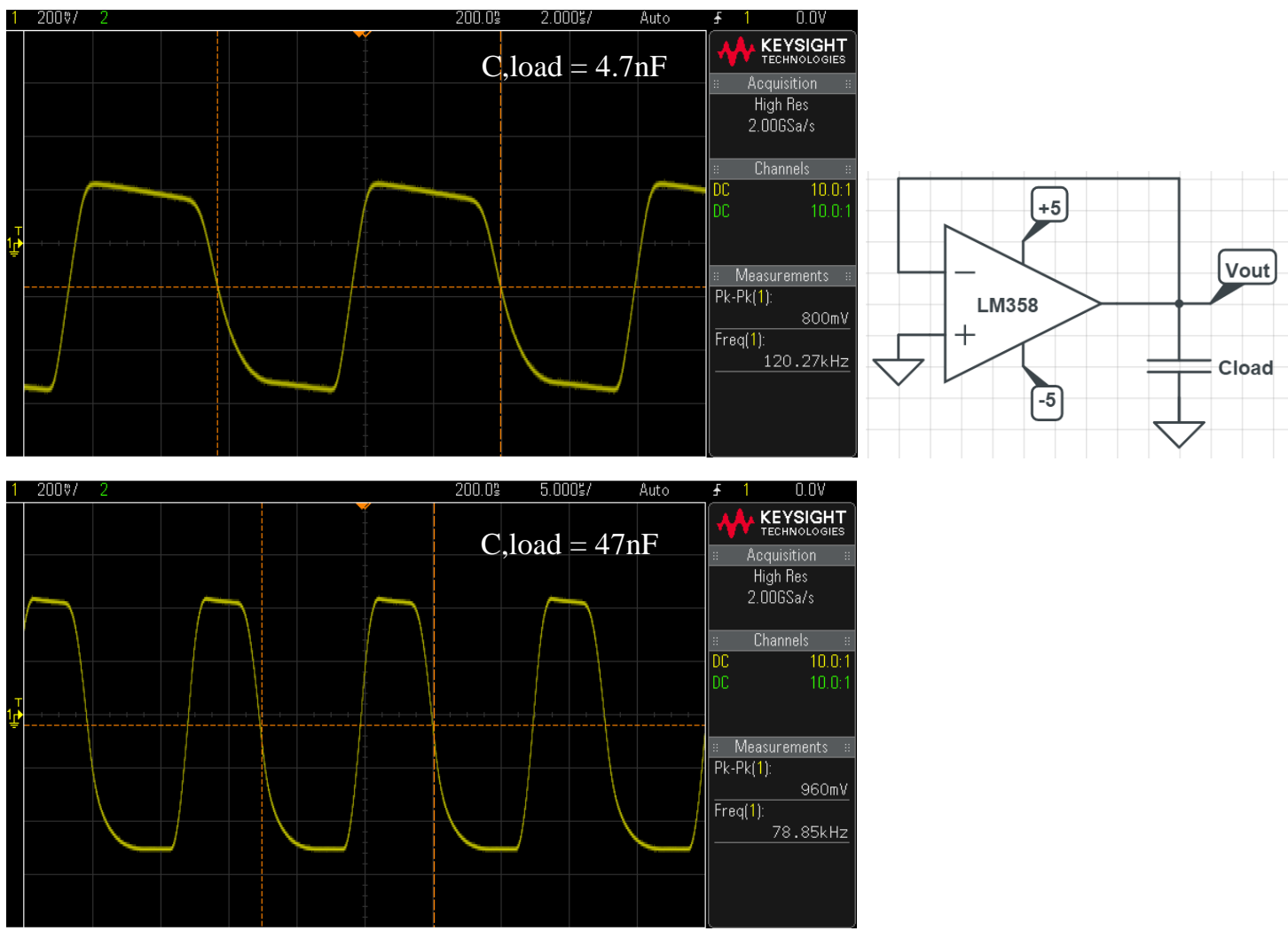

Figure 10. LM358 powered from $\pm 5 \mathrm{~V}$ supply configured as follower with grounded input (above). $\mathrm{V}_{\text {out }}$ is probed with loads $4.7 \mathrm{nF}$ (top left) and $47 \mathrm{nF}$ (bottom left).

Oscilloscope captures show the op-amp attempting to drive $4.7 \mathrm{n}$ and $47 \mathrm{n}$ load capacitances where the output is oscillating with a grounded input. It's unable to maintain a virtual short between the input terminals due to low phase margin, so negative feedback becomes positive feedback.

\section{Characterizing the LM358 Op-Amp}

To characterize gain and bandwidth, the device is configured as a non-inverting amplifier with weak feedback and closed-loop gain of -10, as shown in Figure 6. The input frequency is swept from $1 \mathrm{kHz}$ to $25 \mathrm{kHz}$ and at each data point open-loop gain and GBW are calculated using Equation 2.1 . Below $1 \mathrm{kHz}$, the inverting terminal voltage, $V_{n}$, is imperceptible due to oscilloscope accuracy and above $25 \mathrm{kHz}$, the output becomes too 
distorted for a reliable measurement due to the op-amp's inherent nonlinearity. GBW is averaged over measurements where distortion is minimal.

To characterize $r_{o}$, the device is placed in a voltage follower configuration with varying capacitive loads at the output, as shown in Figure 7. The oscillation frequency is observed which allows $f_{p 2}^{\prime}$ and $r_{o}$ to be calculated using Equations 2.2 and 2.3.
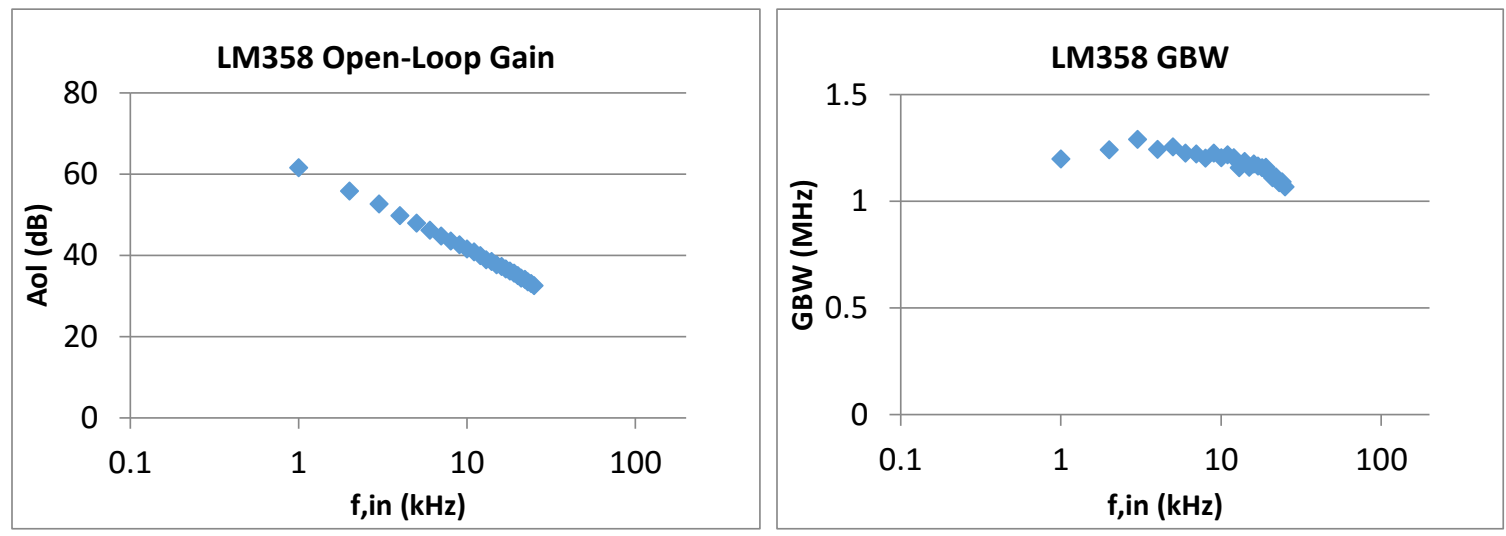

Figure 11. Open loop gain drops at 20db/dec as expected. Average GBW is $1.18 \mathrm{MHz}$.
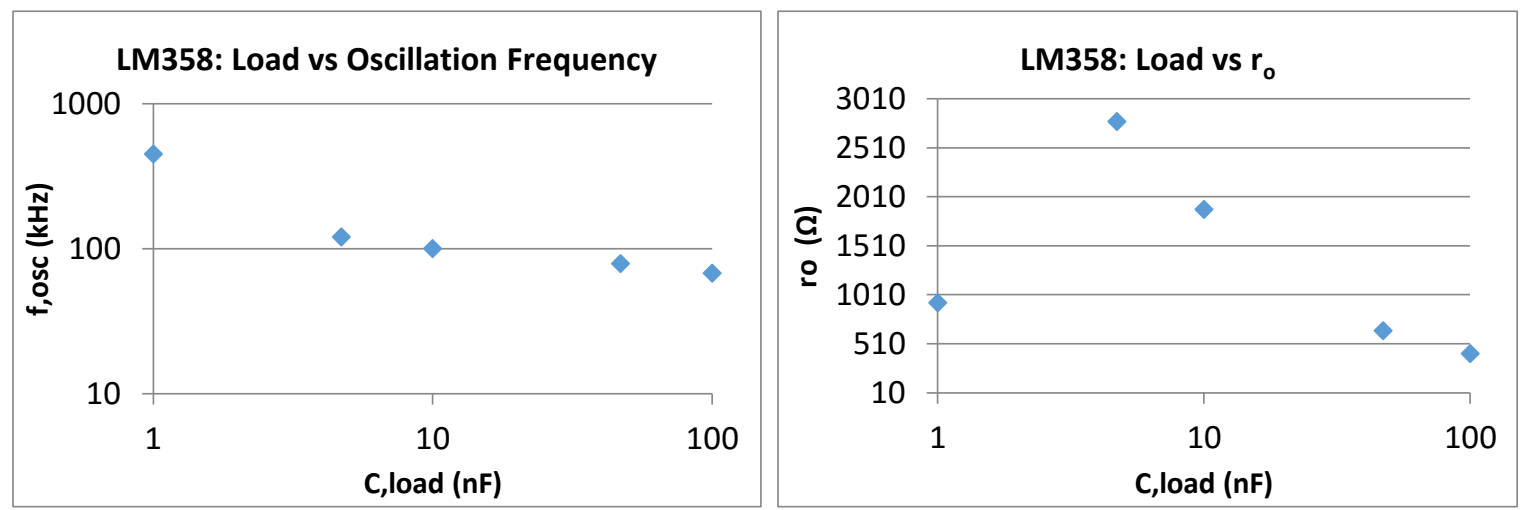

Figure 12. Oscillation frequency and output impedance is much higher than predicted by simulations.

\begin{tabular}{|r|r|r|r|}
\hline C,load (nF) & f,osc $(\mathbf{k H z})$ & \multicolumn{1}{l|}{ f,pole $(\mathbf{k H z})$} & $\boldsymbol{r}_{\boldsymbol{o}}(\boldsymbol{\Omega})$ \\
\hline 1 & 450 & 171.6 & 927 \\
\hline 4.7 & 120 & 12.2 & 2775 \\
\hline 10 & 100 & 8.5 & 1878 \\
\hline 47 & 79.1 & 5.3 & 639 \\
\hline 100 & 67.7 & 3.9 & 410 \\
\hline
\end{tabular}

Table 2 . The secondary pole is displaced to an extremely low frequency. 
The open-loop gain Bode plot shows the expected linear decline in gain versus frequency. GBW fluctuates with a slightly better than expected average of $1.18 \mathrm{MHz}$. However, the observed oscillation frequency, pole frequency, and output resistance are wildly divergent from what simulations had predicted revealing the situation to be much worse than expected.

\subsubsection{LMC662}

Investigating Instability

Simulations below present the op-amp's frequency and step responses.
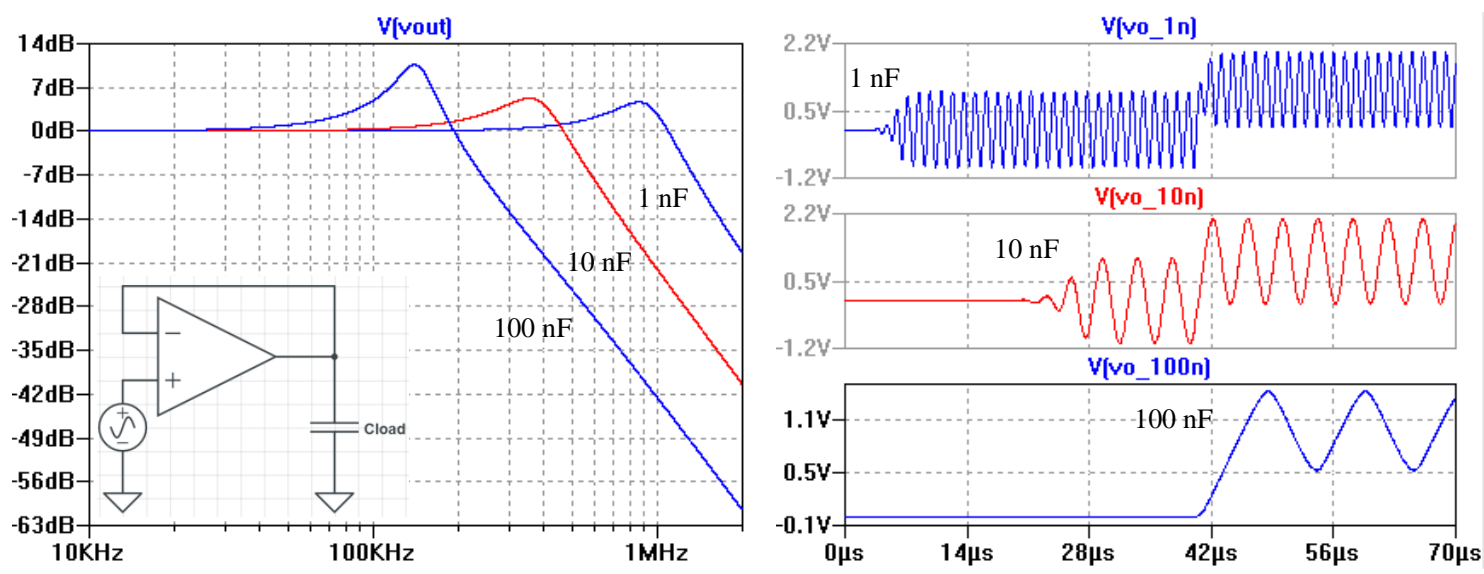

Figure 13. Gain peaks at the loop's natural frequency, at which the ringing oscillates.

An increasing magnitude of instability can be seen over three decades of heavy loading from $1 \mathrm{nF}$ to $100 \mathrm{nF}$. Resonance-like gain peaking in the frequency domain manifests itself as unforced oscillations in time domain where the frequency of ringing is approximately where the peak occurs. The feedback continues to oscillate even after an impulse occurs at $50 \mu \mathrm{s}$. By noting this frequency the parasitic pole and $r_{o}$ can be calculated using Equations 2.2 and 2.3, the results of which are tabulated in Table 3. GBW is assumed to $1.4 \mathrm{MHz}$ as claimed by datasheet. 


\begin{tabular}{|r|r|r|r|}
\hline \multicolumn{1}{|c|}{ C,load (nF) } & f,peak (kHz) & f,pole $(\mathbf{k H z})$ & \multicolumn{1}{c|}{$\boldsymbol{r}_{\boldsymbol{o}}(\boldsymbol{\Omega})$} \\
\hline 1 & 862 & 530.7 & 299.9 \\
\hline 2.2 & 630 & 283.5 & 255.2 \\
\hline 4.7 & 472 & 159.1 & 212.8 \\
\hline 10 & 354.6 & 89.8 & 177.2 \\
\hline 22 & 262 & 49.0 & 147.5 \\
\hline 47 & 193.3 & 26.7 & 126.9 \\
\hline 100 & 140.1 & 14.0 & 113.5 \\
\hline
\end{tabular}

Table 3. Simulation data of a voltage follower with the LMC662 to determine its output impedance.

As the load is made heavier, the geometric gap between $f_{\text {peak }}$ and $f_{\text {pole }}$ gets broader while $r_{o}$ settles toward an idealized value of around $100 \Omega$.

It may sound reasonable to look at Figure 13 and judge the overshoot from a $1 \mathrm{nF}$ load as acceptable, but it would be a misguided approach to solely rely on simulation data. Performing this experiment in a lab with an actual device tells a slightly different story.
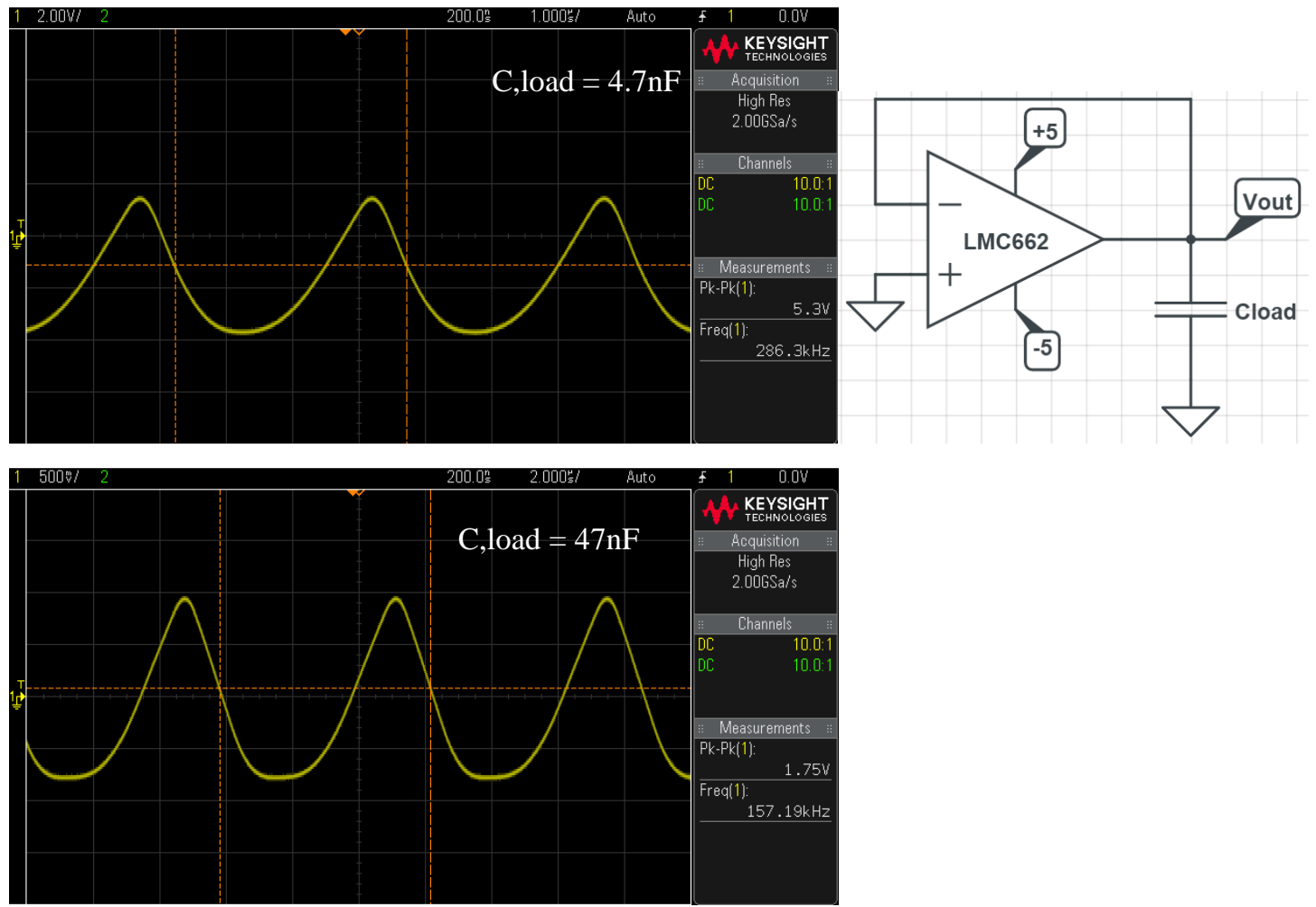

Figure 14. LMC662 powered from $\pm 5 \mathrm{~V}$ supply configured as follower with grounded input (above). $\mathrm{V}_{\text {out }}$ is probed with loads $4.7 \mathrm{nF}$ (top left) and $47 \mathrm{nF}$ (bottom left). 


\section{Characterizing the LMC662 Op-Amp}

Gain and bandwidth are characterized using a non-inverting configuration, as shown in Figure 6. The input frequency is swept from $1 \mathrm{kHz}$ to $1.4 \mathrm{MHz}$ (claimed GBW) and open-loop gain and GBW are calculated using Equation 2.1. GBW is averaged over measurements where distortion is minimal. To characterize $r_{o}$, the device is placed in a voltage follower configuration with varying capacitive loads at the output, as shown in Figure 7. The oscillation frequency is observed which allows $f_{p 2}^{\prime}$ and $r_{o}$ to be calculated using Equations 2.2 and 2.3.
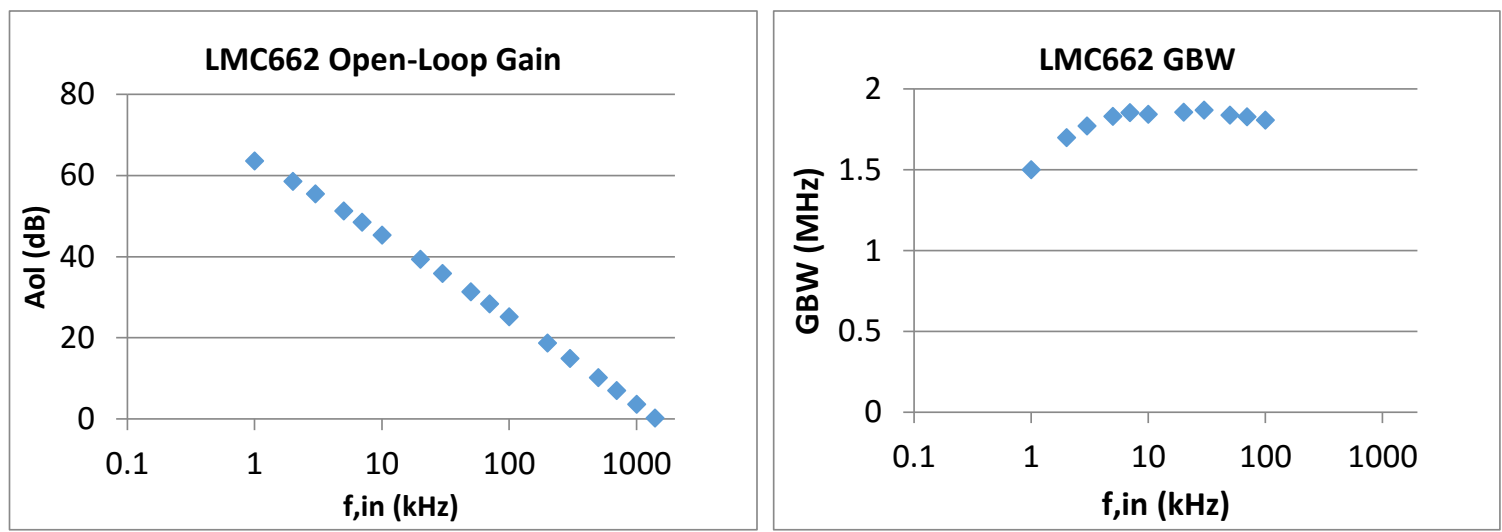

Figure 15. Open loop gain drops at $20 \mathrm{db} / \mathrm{dec}$ as expected. Average GBW is $1.79 \mathrm{MHz}$.
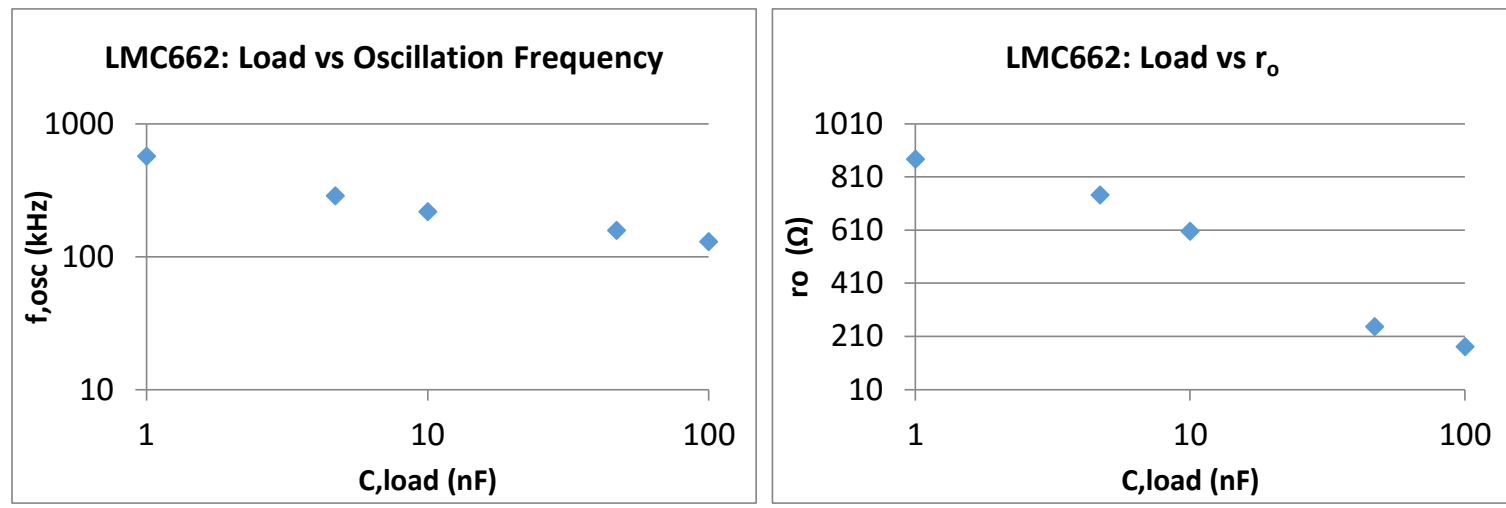

Figure 16. Oscillation frequency and output impedance is much higher than predicted by simulations. 


\begin{tabular}{|r|r|r|r|}
\hline C,load (nF) & f,osc (kHz) & f,pole $(\mathbf{k H z})$ & $\boldsymbol{r}_{\boldsymbol{o}}(\mathbf{\Omega})$ \\
\hline 1 & 570 & 181.5 & 877 \\
\hline 4.7 & 286 & 45.7 & 741 \\
\hline 10 & 217 & 26.3 & 605 \\
\hline 47 & 157 & 13.8 & 246 \\
\hline 100 & 129 & 9.3 & 171 \\
\hline
\end{tabular}

Table 4. The secondary pole is displaced to an extremely low frequency.

The open-loop gain Bode plot shows the expected linear decline in gain versus frequency. GBW is relatively flat over frequency with a better than expected average of 1.79 MHz. However, the observed oscillation frequency, pole frequency, and output resistance are very different from what simulations had predicted revealing the situation to be much worse than expected.

\subsubsection{LTC6084}

\section{$\underline{\text { Investigating Instability }}$}

Simulations below present the op-amp's frequency and step responses.
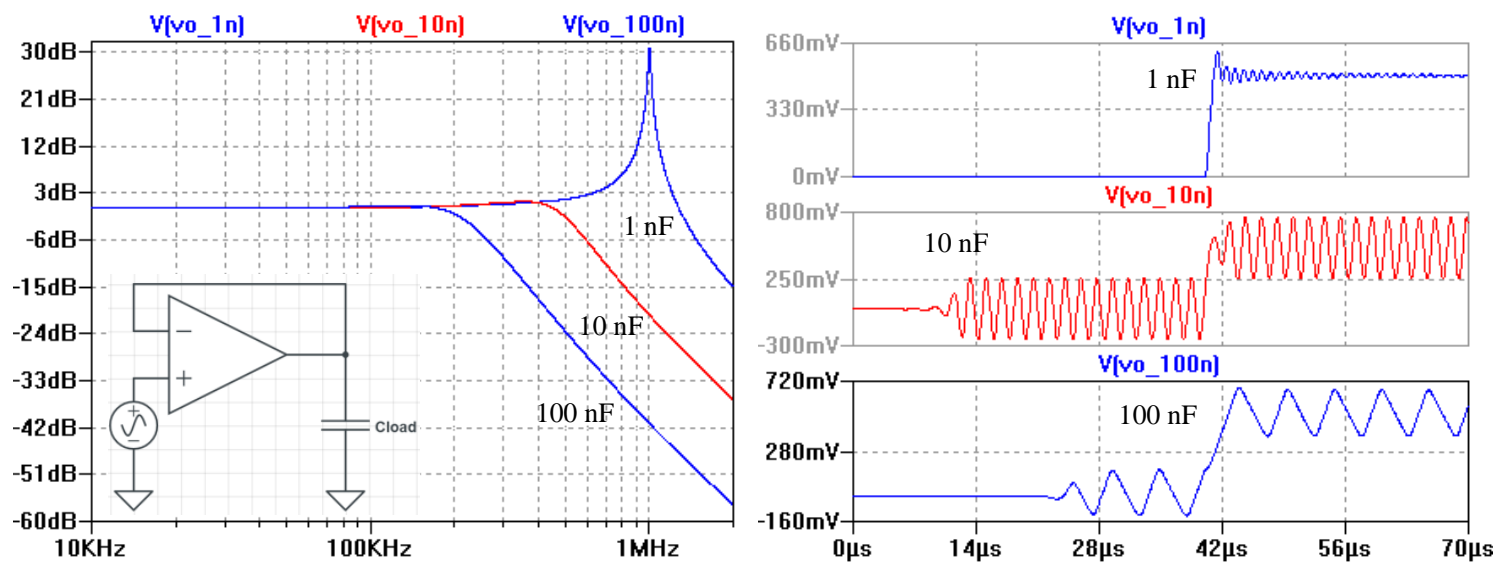

Figure 17. Gain peaks at the loop's natural frequency, at which the ringing oscillates.

The transient simulation shows an increasing magnitude of instability can be seen over three decades of heavy loading from $1 \mathrm{nF}$ to $100 \mathrm{nF}$. On the contrary, only the $1 \mathrm{nF}$ load exhibits gain peaking in the frequency domain while the other two have a slight hump, yet their feedback continues to oscillate even after an impulse occurs at $50 \mu \mathrm{s}$. By noting this frequency the parasitic pole and $r_{o}$ can be calculated using Equations 2.2 and 2.3, the 
results of which are tabulated in Table 5. GBW is assumed to $1.5 \mathrm{MHz}$ as claimed by datasheet.

\begin{tabular}{|r|r|r|r|}
\hline \multicolumn{1}{|c|}{ C,load (nF) } & f,peak (kHz) & f,pole $(\mathbf{k H z})$ & $\boldsymbol{r}_{\boldsymbol{o}}(\mathbf{\Omega})$ \\
\hline 1 & 1000 & 666.7 & 238.7 \\
\hline 2.2 & 739 & 364.1 & 198.7 \\
\hline 4.7 & 521 & 181.0 & 187.1 \\
\hline 10 & 356 & 84.5 & 188.4 \\
\hline 22 & 240 & 38.4 & 188.4 \\
\hline 47 & 172 & 19.7 & 171.7 \\
\hline 100 & 133 & 11.8 & 135.0 \\
\hline
\end{tabular}

Table 5. Simulation data of a voltage follower with the LTC6084 to determine its output impedance.

As the load is made heavier, the geometric gap between $f_{\text {peak }}$ and $f_{\text {pole }}$ gets broader while $r_{o}$ settles toward a possibly idealized value of around $100 \Omega$.

It may sound reasonable to look at Figure 17 and judge the ringing from a $1 \mathrm{nF}$ load as acceptable, but it would be a misguided approach to solely rely on simulation data. Performing this experiment in a lab with an actual device tells a different story.
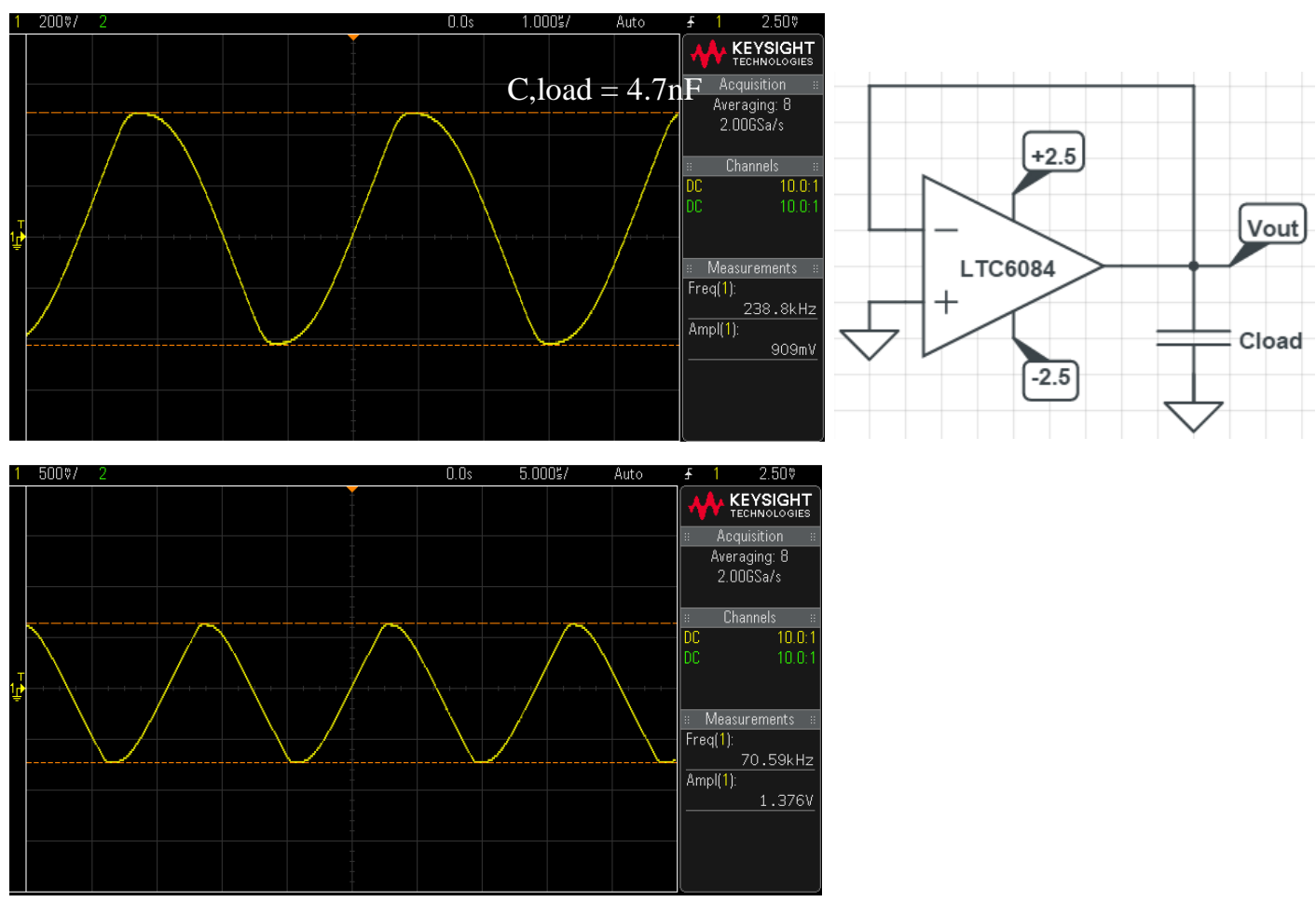

Figure 18 . LTC6084 powered from $\pm 2.5 \mathrm{~V}$ supply configured as follower with grounded input (above). $\mathrm{V}_{\text {out }}$ is probed with loads $4.7 \mathrm{nF}$ (top left) and $47 \mathrm{nF}$ (bottom left). 


\section{Characterizing the LTC6084 Op-Amp}

Gain and bandwidth are characterized using a non-inverting configuration, as shown in Figure 6. The input frequency is swept from $1 \mathrm{kHz}$ to $1.5 \mathrm{MHz}$ (claimed GBW) and open-loop gain and GBW are calculated using Equation 2.1. GBW is averaged over measurements where distortion is minimal. To characterize $r_{o}$, the device is placed in a voltage follower configuration with varying capacitive loads at the output, as shown in Figure 7. The oscillation frequency is observed which allows $f_{p 2}^{\prime}$ and $r_{o}$ to be calculated using Equations 2.2 and 2.3.
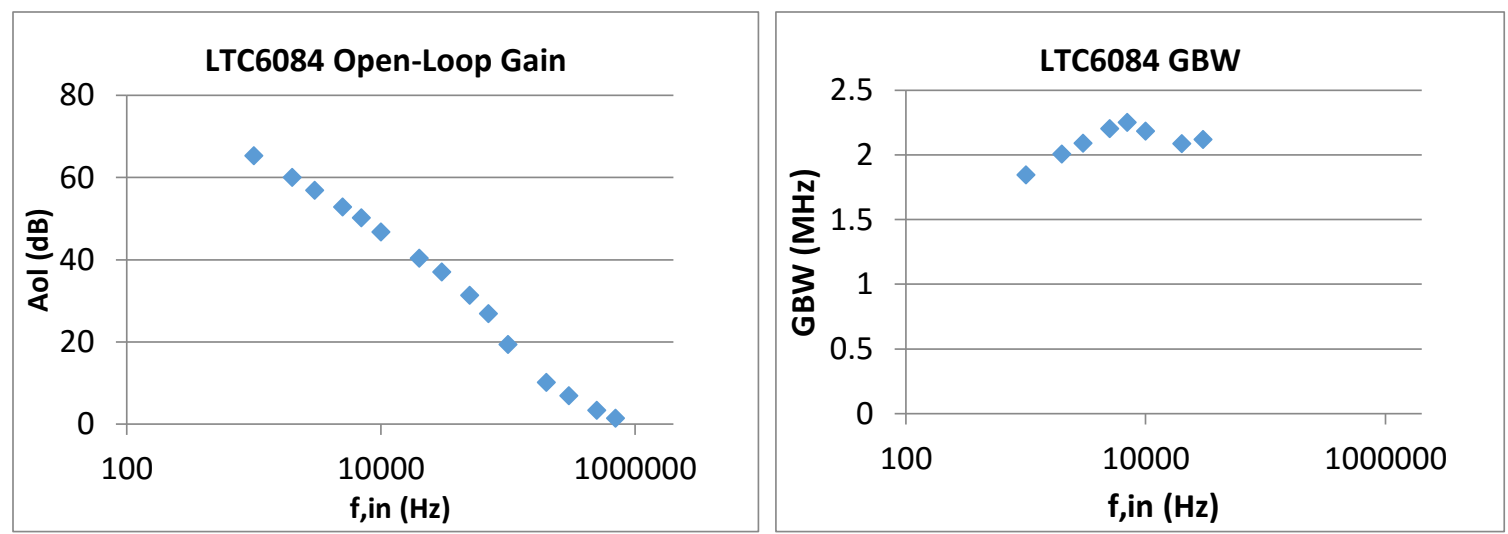

Figure 19. Open loop gain drops at 20db/dec as expected. Average GBW is $2.1 \mathrm{MHz}$.
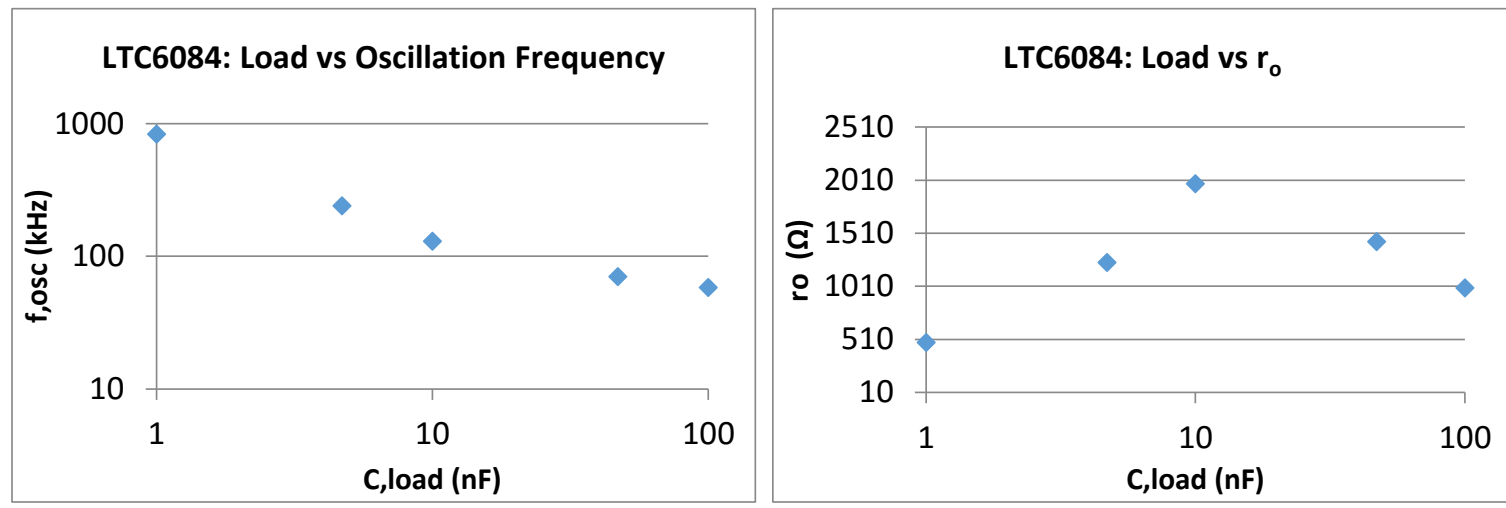

Figure 20. Oscillation frequency and output impedance is much higher than predicted by simulations. 


\begin{tabular}{|r|r|r|r|}
\hline C,load (nF) & f,osc (kHz) & f,pole $(\mathbf{k H z})$ & $\boldsymbol{r}_{\boldsymbol{o}}(\mathbf{\Omega})$ \\
\hline 1 & 834 & 331.2 & 481 \\
\hline 4.7 & 240 & 27.4 & 1235 \\
\hline 10 & 130 & 8.0 & 1978 \\
\hline 47 & 70.5 & 2.4 & 1431 \\
\hline 100 & 58 & 1.6 & 994 \\
\hline
\end{tabular}

Table 6. The secondary pole is displaced to an extremely low frequency.

The open-loop gain Bode plot shows the expected linear decline in gain versus frequency. GBW is relatively flat over frequency with a better than expected average of 2.1 MHz. However, the observed oscillation frequency, pole frequency, and output resistance are very different from what simulations had predicted revealing the situation to be much worse than expected.

\subsection{Summarized Results and Discussion}

Follower and gain circuits assembled using three different op-amps were simulated and tested. All three performed better in terms of GBW than what was claimed in their respective datasheets, but driving capacitive loads had wildly different results between experiment and simulation. The table below summarizes some of the more interesting data collected in the previous section.

\begin{tabular}{|l|cc|cc|cc|c|}
\hline & & & \multicolumn{2}{|c|}{$\mathbf{C L}=\mathbf{1} \mathbf{~ n F}$} & \multicolumn{2}{c|}{$\mathbf{C L}=\mathbf{1 0} \mathbf{n F}$} & \\
\hline Op-amp & $\begin{array}{l}\boldsymbol{f}_{\boldsymbol{u}}(\mathbf{M H z}) \\
(\text { nominal) }\end{array}$ & $\begin{array}{c}\boldsymbol{f}_{\boldsymbol{u}}(\mathbf{M H z}) \\
(\mathbf{m e a s u r e d})\end{array}$ & $\begin{array}{c}\boldsymbol{r}_{\boldsymbol{o}}(\mathbf{\Omega}) \\
\text { simulated }\end{array}$ & $\begin{array}{c}\boldsymbol{r}_{\boldsymbol{o}}(\mathbf{\Omega}) \\
\text { measured }\end{array}$ & $\begin{array}{c}\boldsymbol{r}_{\boldsymbol{o}}(\mathbf{\Omega}) \\
\text { simulated }\end{array}$ & $\begin{array}{c}\boldsymbol{r}_{\boldsymbol{o}}(\mathbf{\Omega}) \\
\text { measured }\end{array}$ & $\begin{array}{c}\mathbf{C L} \\
\text { rating }\end{array}$ \\
\hline LM358 & 1.1 & 1.2 & 192 & 927 & 57 & 1878 & $50 \mathrm{pF}$ \\
\hline LMC662 & 1.4 & 1.79 & 300 & 877 & 177 & 605 & $100 \mathrm{pF}$ \\
\hline LTC6084 & 1.5 & 2.1 & 239 & 481 & 188 & 1978 & $150 \mathrm{pF}$ \\
\hline
\end{tabular}

Table 7. Expected vs. mesaured values of GBW and output imepdance (for 1 and $10 \mathrm{nF}$ loads only).

The measured values of the perceived output impedance were a lot higher than expected. More than anything, it highlights the fact SPICE models and simulations can be unreliable in certain situations. These op-amps are rated to drive up to a hundred or so pF's stably with tolerable overshoot, so even a $1 \mathrm{nF}$ load is extremely heavy.

It is important to recognize that SPICE assumes perfect linearity especially in AC or frequency response simulations. Yet the oscilloscope captures in Figure 10, Figure 14, 
and Figure 18 illustrate largely nonlinear behavior, which has small effect on measuring the true value of gain or output impedance. To make matters worse, real circuits are also affected by voltage supply, temperature, wiring/routing, supply noise, and many others that simulations do not normally account for all together. It was noticed that the output oscillation frequency varied slightly if the supply was changed from $5 \mathrm{~V}$ to $10 \mathrm{~V}$, for example, but this was not the case in simulations.

But to allay any concerns, the frequency compensation techniques developed in subsequent chapters are relatively forgiving and cooperative with inaccuracy in measurements. 


\section{Chapter 3: Modeling the System}

\subsection{Review of Relevant Feedback Control Theory}

Before diving into a model that allows an analytical study of feedback instability, an overview of relevant feedback circuit theory may be useful. Figure 21 illustrates a general feedback control system where $\mathrm{A}(\mathrm{s})$ and $\mathrm{F}(\mathrm{s})$ are the feedforward and feedback networks, respectively.

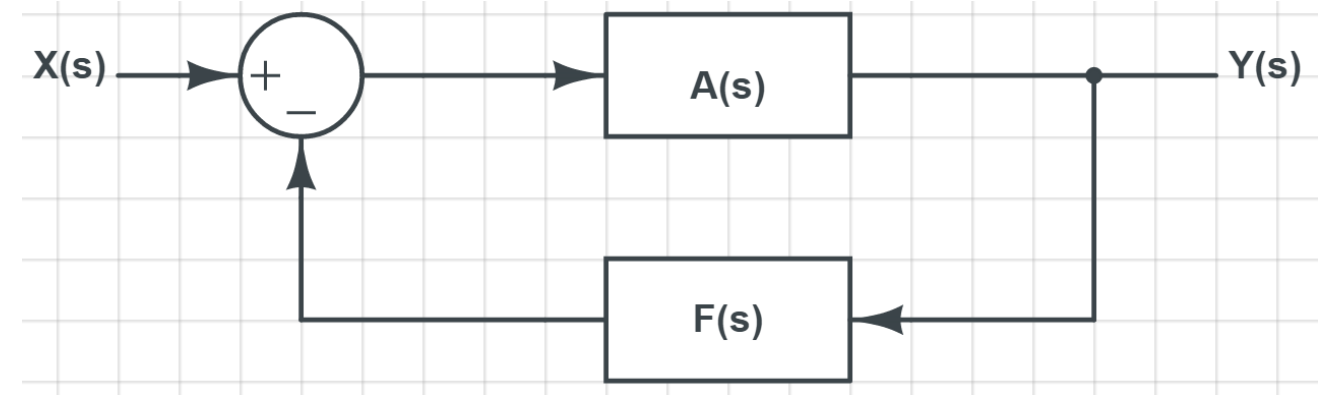

Figure 21. Generic controls system with A(s) forwardforward and F(s) feedback.

Since the output of $F(s)$ is equal to $F(s) Y(s)$, the summing junction creates an error signal by subtracting $F(s) Y(s)$ from $X(s)$. Then, $Y(s)$ is the product of the error signal and $A(s)$.

$$
\begin{gathered}
E(s)=X(s)-F(s) Y(s) \\
Y(s)=A(s)[X(s)-F(s) Y(s)]
\end{gathered}
$$

Rearranging the second equation, the closed-loop transfer function can be obtained.

$$
H(s)=\frac{Y(s)}{X(s)}=\frac{A(s)}{1+A(s) F(s)}
$$

$\mathrm{A}(\mathrm{s})$ is typically defined as the open-loop transfer function, where if the loop is broken then $\mathrm{G}(\mathrm{s})=0$, then $\mathrm{H}(\mathrm{s})=\mathrm{A}(\mathrm{s})$. In a stable system, the error term is minimized because the output of $\mathrm{G}(\mathrm{s})$ is identical to the input signal, which would imply the system output is also identical to the input. Note that the denominator of $\mathrm{H}(\mathrm{s})$ in this form is known as the characteristic equation when set equal to 0 . It can be used to find the system poles, natural frequency, and damping ratio.

Applying these principles to op-amp circuits, the feedforward gain must be very high to ensure the closed loop transfer characteristic with negative feedback is approximately independent of op-amp gain. In other words, feedback provides gain 
desensitization so closed loop gain is insensitive to open-loop gain. This is more apparent at low frequencies when the closed loop transfer function becomes

$$
H=\frac{A_{0}}{1+A_{0} \beta} \approx \frac{1}{\beta}\left(1-\frac{1}{A_{0} \beta}\right)
$$

where $A_{0}$ and $\beta$ are the $\mathrm{DC}$ gains of $\mathrm{A}(\mathrm{s})$ and $\mathrm{F}(\mathrm{s})$, respectively. When $A_{0}$ is very large the closed-loop transfer will be approximately $1 / \beta$. Even if $A_{0}$ varies by a factor of $2, H$ is only affected by a small percentage because $1 / A_{0} \beta \ll 1$.

However, this is hardly the case at higher frequencies because the feedforward amplifier has a single-pole response as given below, where $\omega_{o}$ is the $-3 \mathrm{db}$ frequency associated with an op-amps dominant pole.

$$
A(s)=\frac{A_{0}}{1+s / \omega_{o}}
$$

Using this, the closed-loop transfer function can be expressed for high frequency gain.

$$
H(s)=\frac{\frac{A_{0}}{1+s / \omega_{o}}}{1+\beta \frac{A_{0}}{1+s / \omega_{o}}}=\frac{\frac{A_{0}}{1+\beta A_{0}}}{1+\frac{S}{\left(1+\beta A_{0}\right) \omega_{o}}}
$$

The denominator provides the pole location at $\left(1+\beta A_{0}\right) \omega_{o}$ which has now increased by a factor of $\left(1+\beta A_{0}\right)$ compared to the open-loop pole. The extension in bandwidth does come at the cost of a proportional reduction in loop gain such that the gain-bandwidth product remains constant for an op-amp.

Another important property of closing the loop with negative feedback is nonlinearity suppression. Nonlinearity can be regarded as variation in the small signal transconductance or voltage gain with respect to the input swing or DC level. Because negative feedback keeps closed-loop gain constant and independent of open-loop gain, distortion from any change in a transistor's or amplifier V/I transfer curve is reduced.

\subsection{Summing Junction and Integrator Model of an Op-Amp}

To model the closed-loop system with a parasitic pole, the voltage follower configuration is used as it presents the worst case scenario for stability. This is because a feedback factor of $\beta=1$ offers the strongest possible feedback. 


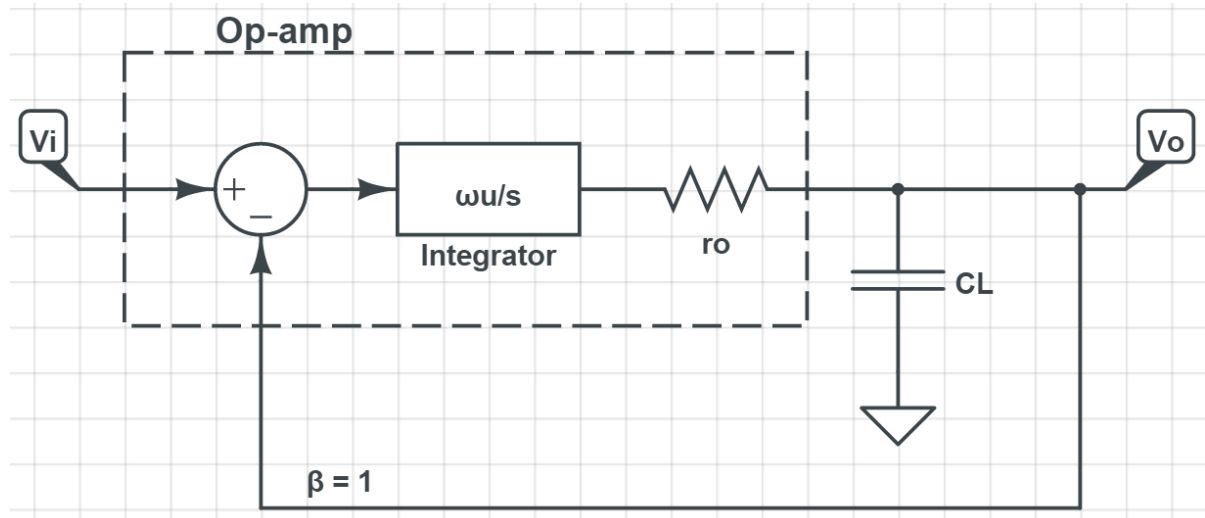

Figure 22. Op-amp modeled as smming junction, integrator, and output impedance.

A very large open-loop DC gain $A_{D C}$ is assumed so the closed-loop gain is $1 / \beta \cong 1$ for a follower. The op-amp is taken to be a summing junction, integrator with a bandwidth of $\omega_{u}$, and output impedance $r_{o}$ loaded by $C_{L}$ around which the loop is closed. This forms a second order control loop because there are two integrators: the op-amp and $C_{L}$. Figure 22 illustrates an idealized op-amp model that is used for the present argument. It is derived and discussed in greater detail in the Appendix.

Since $r_{o}$ and $C_{L}$ have a transfer characteristic of their own, they can be put into a separate cascade Laplace block, where

$$
T(s)=\frac{1}{r_{o} C_{L} s+1}
$$

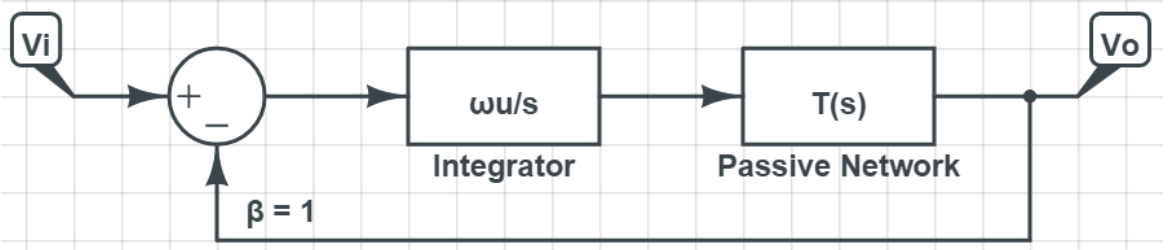

Figure 23. Ouput impedance and load are lumped into a transfer block.

It is known that the transfer function of two cascaded Laplace blocks is the product of their individual transfer functions, so the feedforward transfer can be easily found: $A(s)=\frac{\omega_{u}}{s} * T(s)$. Then, as derived in $\S 3.1$, the closed-loop transfer function can be written as 


$$
H(s)=\frac{A(s)}{1+A(s) F(s)}=\frac{\frac{\omega_{u}}{s} \frac{1}{r_{o} C_{L} s+1}}{1+\frac{\omega_{u}}{s} \frac{1}{r_{o} C_{L} s+1}}
$$

From this transfer function, the characteristic equation of this control loop is apparent

$$
1+\frac{\omega_{u}}{s} \frac{1}{r_{o} C_{L} s+1}=0
$$

Rearranging the equation and comparing it to the standard form clearly reveals a second order system.

$$
s^{2}+\frac{s}{r_{o} C_{L}}+\frac{\omega_{u}}{r_{o} C_{L}}=0 \Leftrightarrow s^{2}+2 \zeta \omega_{n} s+\omega_{n}^{2}=0
$$

Now, the poles, damping ratio, and natural frequency can easily be extracted.

$$
\begin{gathered}
p_{1}, p_{2}=-\frac{1}{2 r_{o} C_{L}} \pm \sqrt{\frac{1}{4 r_{o}^{2} C_{L}^{2}}-\frac{\omega_{u}}{r_{o} C_{L}}} \\
\omega_{n}=\sqrt{\frac{\omega_{u}}{r_{o} C_{L}}} \\
\zeta=\frac{1}{2 \sqrt{r_{o} C_{L} \omega_{u}}}
\end{gathered}
$$

Interestingly, the natural frequency equation 3.12 is the same as equation 2.3 that predicts where the op-amp will oscillate except with $f_{p 2}^{\prime}=\frac{1}{2 \pi r_{o} C_{L}}$ is substituted in and rearranged. Fortunately, both natural frequency and damping ratio depend on the same measurable or known quantities. Once $r_{o}$ has been estimated from the measured oscillation frequency $\left(\omega_{n}\right.$ or $\left.f_{n}\right)$, the system can be shown to be underdamped $(\zeta<1)$.

Revisiting the characteristic equation standard form in equation 3.10, it describes a classical second-order homogenous system for which the poles are given by

$$
p_{1}, p_{2}=-\zeta \omega_{n} \pm \omega_{n} \sqrt{\zeta^{2}-1}
$$

where if $\zeta<1$ the poles form a complex conjugate pair. Figure 24 illustrates, on a polezero plot, the relationship between the damping ratio and how complex the poles become. They lie in a semi-circle about the origin with a radius defined by $\omega_{n}$ and the angle by $\zeta$. 


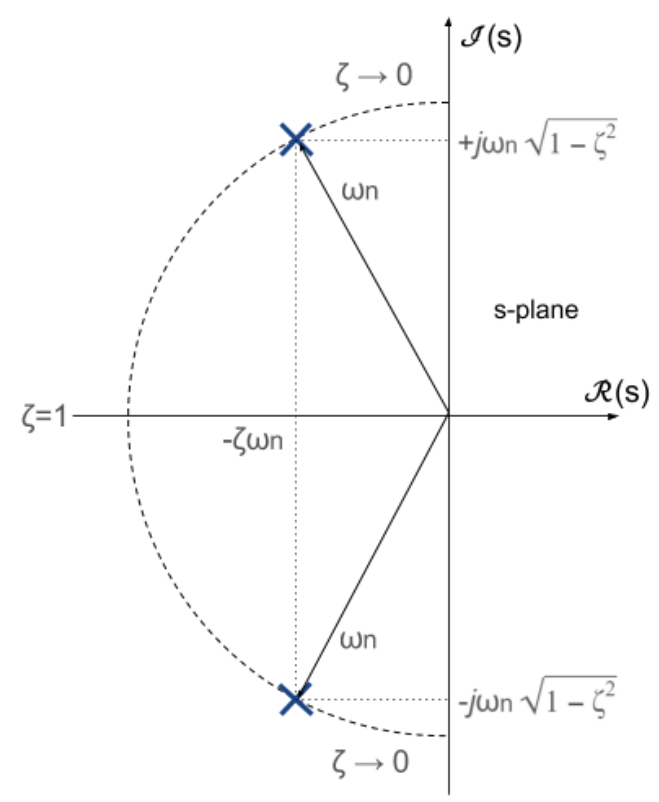

Figure 24. Poles become more complex as damping ratio decreases.

Jumping back to op-amps, frequency response can also be viewed on a pole-zero plot where, by design, the two main poles are set very far apart on the real-axis. However, with heavy capacitive loading, the secondary pole to the right gets pulled back and split into a pair of complex poles, as illustrated by the system's root locus in Figure 25. They then begin to cause oscillatory behavior parasitic to the control loop.

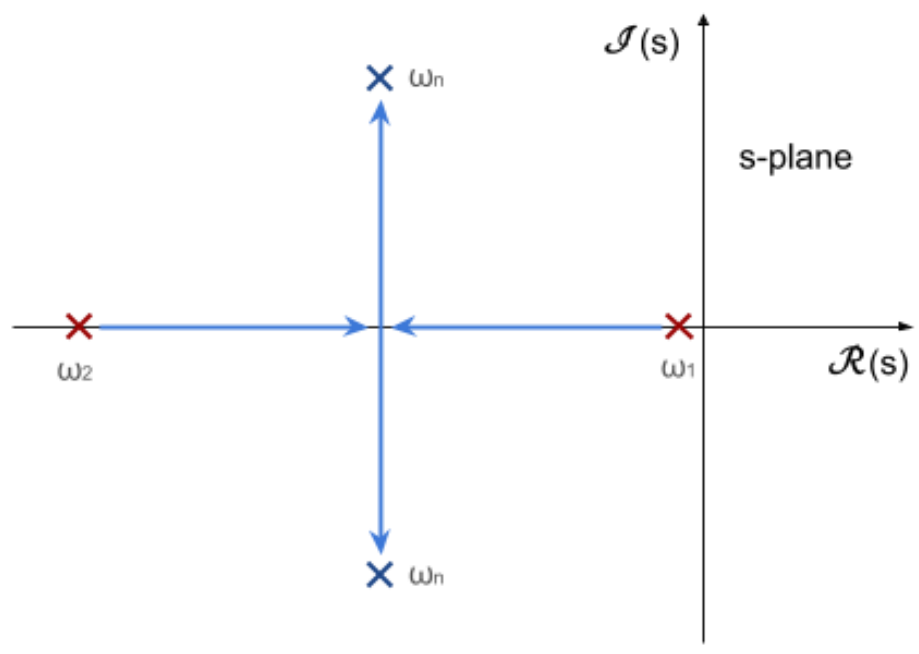

Figure 25. Root locus of op-amp modeled as a control system. Dominant and secdonary poles come together and become complex. 


\subsection{Compensating the Feedback}

Op-amp characterization methods have been outlined to allow calculation of transfer and loop characteristics. It has been shown that using the instability oscillation frequency $\left(f_{n}\right.$ or $\left.f_{\text {osc }}\right) r_{o}$ and $\zeta$ can be estimated for a given device with feedback. So the challenge remains of the how the system can be modified toward a critically damped $(\zeta=1)$ state. The modular nature of the control loop does however serve a possible remedy. The contents of $T(s)$ are more easily accessible than the other two elements of the loop (Figure 23), and currently $r_{o}$ and $C_{L}$ occupy this Laplace block.

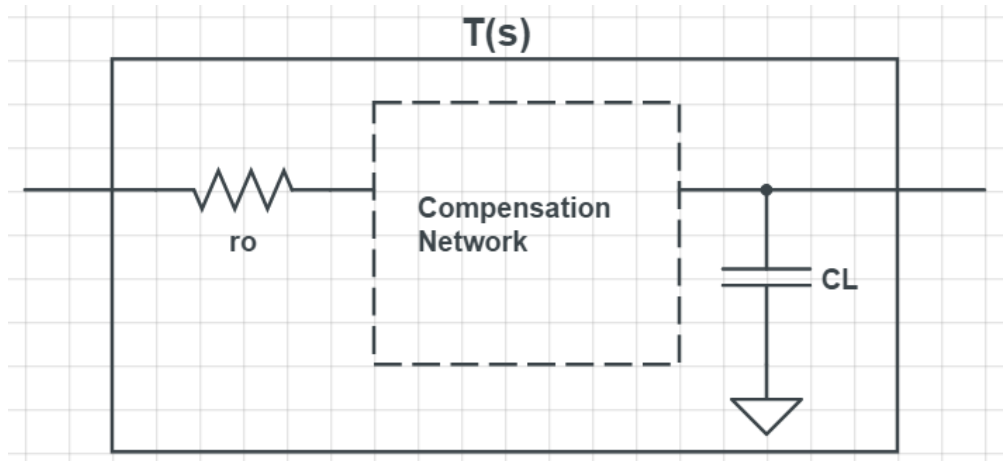

Figure 26. T(s) can be supplemented with a compensation network that could stabalize feedback.

Zooming in on $T(s)$, it lends itself to one possible class of solutions where a frequency compensation network is inserted between the op-amp and load. The rest of this thesis is dedicated to further developing this block and possible R-C networks that can be used.

Chapter 4 will present the commonly used method of using a shunt resistor to lower the output impedance, as well as the complementary method of using a series capacitor to lower the load capacitance, and generalize them into a composite method that uses both. Relevant loop parameters will be derived and selecting right values will be discussed. Chapter 5 is a step-by-step guide to designing this compensation network that considers tradeoffs. Finally, Chapter 6 summarizes results of simulations and lab experiments of the composite compensation network successfully being used with the opamps from Chapter 2. 


\section{Chapter 4: Proposed Solutions}

\subsection{Method 1: Shunt Resistor}

Placing a shunt resistor at the output is a commonly used tactic in an attempt to lower the op-amp's output impedance. This section will analyze its effect on a closedloop system, look at how the open-loop response is affected, and show a practical implementation.

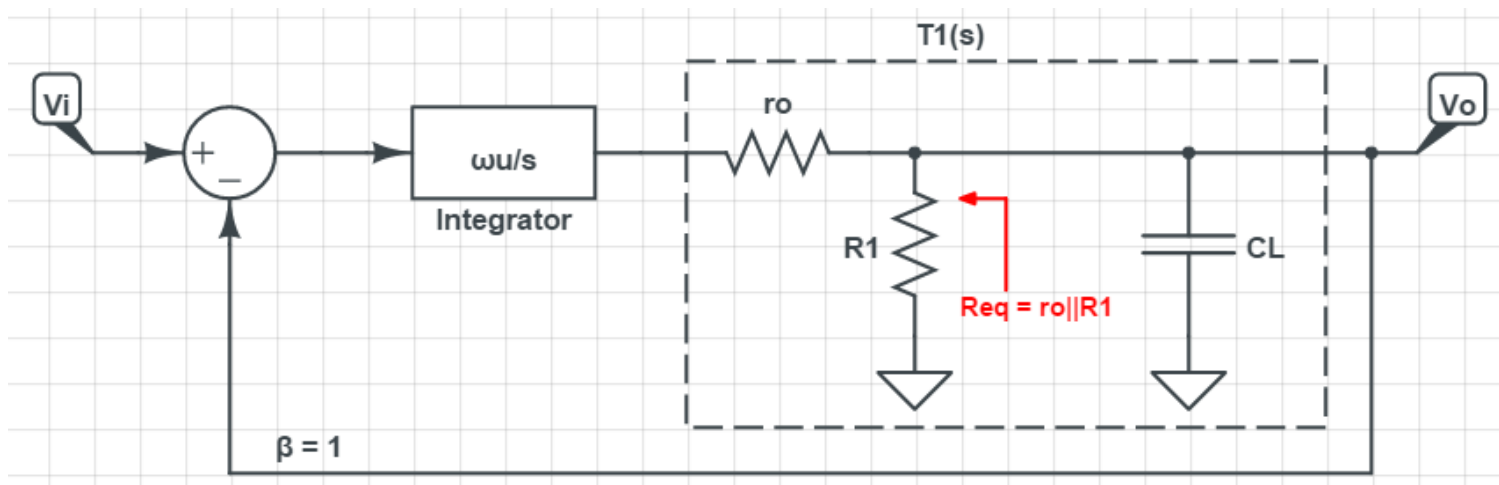

Figure 27. A resitor shunted to ground at the op-amp output biases the output stage with more current.

The compensation network $T_{1}$ transfer characteristic is given below. Comparing it with Equation 3.7 (without $R_{1}$ ), it is obvious that $R_{1}$ will influence the closed-loop.

$$
T_{1}(s)=\frac{R_{1}}{R_{1} r_{0} C_{L} s+R_{1}+r_{o}}
$$

\subsubsection{Closed-Loop Analysis with Example Application}

Looking into the output, the equivalent resistance seen is $r_{o} \| R_{1}$, as illustrated in Figure 27. At the device level, the shunt resistor actually biases the output stage at a larger DC current, which increases its transconductance but reduces its output impedance to maintain a buffer gain $g_{m} r_{o}$ of 1 . However, $r_{o}$ and $R_{1}$ form a voltage divider which ends up changing loop dynamics. The new characteristic equation from the closed-loop transfer function, rearranged into standard form, is given below.

$$
s^{2}+\frac{\left(R_{1}+r_{o}\right)}{R_{1} r_{0} C_{L}} s+\frac{\omega_{u}}{r_{o} C_{L}}=0
$$


From here, it is straightforward to extract once again the poles, natural frequency, and damping ratio.

$$
\begin{gathered}
p_{1}, p_{2}=-\frac{\left(R_{1}+r_{o}\right)}{2 R_{1} r_{o} C_{L}} \pm \sqrt{\frac{\left(R_{1}+r_{o}\right)^{2}}{4 R_{1}^{2} r_{o}^{2} C_{L}^{2}}-\frac{\omega_{u}}{r_{o} C_{L}}} \\
\omega_{n}=\sqrt{\frac{\omega_{u}}{r_{o} C_{L}}} \\
\zeta=\frac{\left(1+\frac{r_{o}}{R_{1}}\right)}{2 \sqrt{r_{o} C_{L} \omega_{u}}}
\end{gathered}
$$

Equations 4.3 through 4.5 reveal a few very important outcomes. The introduction of $R_{1}$ has no effect the natural frequency and damping ratio is now tunable through $R_{1}$, as compared with Equations 3.12 and 3.13. Obtaining well-behaved feedback then becomes a matter of selecting the proper value for $R_{1}$.

Consider a real-world application where a device has been characterized with $r_{o}=100 \Omega, f_{u}=1 \mathrm{MHz}$, and $C_{L}=50 \mathrm{nF}$. From Equation 3.13, it can be shown that $\zeta \ll 1:$

$$
\zeta=\frac{1}{2 \sqrt{100 * 50 * 10^{-9} * 2 \pi * 10^{6}}}=0.0892
$$

A real circuit so underdamped is likely to exhibit sustained oscillations. Then, with $R_{1}$ added in, $\zeta$ can be se to 1 for a critically damped system, or to 0.707 for a Butterworth response with a maximally flat passband (frequency response). Setting $\zeta=0.707$ in Equation 4.5, an appropriate value for $R_{1}$ can be obtained:

$$
0.707=\frac{\left(1+\frac{100}{R_{1}}\right)}{2 \sqrt{100 * 50 * 10^{-9} * 2 \pi * 10^{6}}} \Rightarrow R_{1}=14.4 \Omega
$$

Although it is not practical to load an op-amp with such a small-valued resistor (something that will be dealt with in a later section), this will dampen out the natural frequency with minimal overshoot in the impulse response. 


\subsubsection{Open-Loop Analysis with Example Application}

Opening the loop, only the feedforward (loop forward gain) remains, but it is enough to evaluate system stability with simple feedback present. Recall that $A(s)=$ $\frac{\omega_{u}}{s} * T(s)$, the loop gain can be written using the compensation network $T_{1}(s)$ :

$$
\begin{aligned}
A_{1}(s) & =\frac{\omega_{u}}{s} * \frac{R_{1}}{R_{1} r_{0} C_{L} s+R_{1}+r_{o}} \\
& =\frac{\omega_{u}}{s} * \frac{R_{1}}{R_{1}+r_{o}} * \frac{1}{\left(R_{1}|| r_{0}\right) C_{L} s+1}
\end{aligned}
$$

From the open-loop transfer function, it can be seen that introducing $R_{1}$ into the circuit shifts the parasitic (secondary) pole to a higher frequency $\frac{1}{\left(R_{1} \| r_{0}\right) C_{L}}$ at the cost of reduced gain by a factor of $\frac{R_{1}}{R_{1}+r_{o}}$. If the pole has been shifted far enough, unity-gain will occur at $\frac{\omega_{u}}{1+r_{o} / R_{1}}$, as illustrated in Figure 28 below.

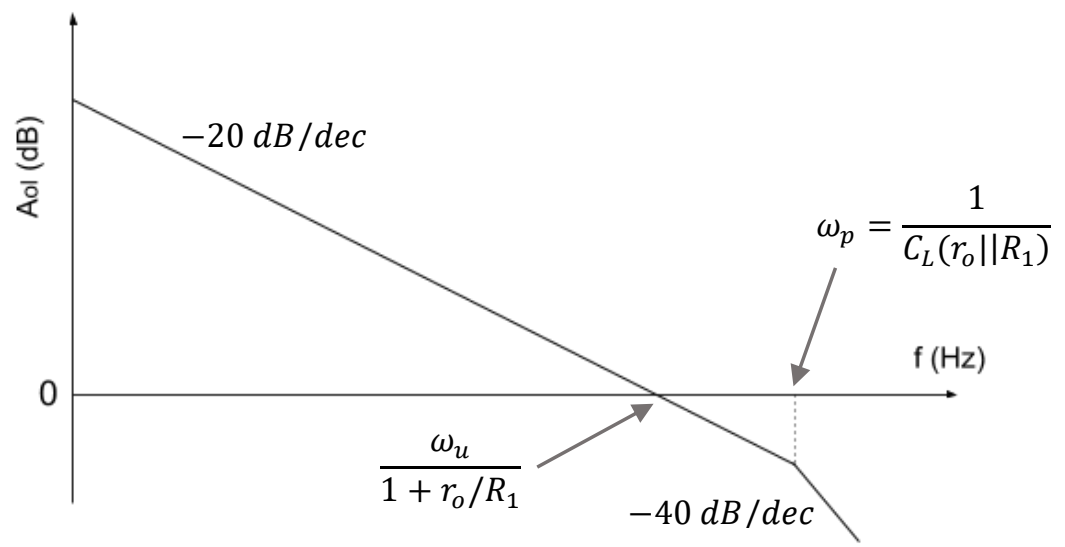

Figure 28. Secondary pole is shifted out, but open-loop gain is reduced.

When feedback is applied, a second-order type-I control system is formed (two poles, one integrator). With one pole near the origin, and as long as the other occurs beyond the unity-gain, phase margin is greater than $45^{\circ}$ and stability is maintained. The preferred location for the second pole is at twice the unity-gain frequency, which results in a Butterworth response. This constraint can be realized by deriving a closed-form expression for $R_{1}$.

$$
\frac{1}{\left(R_{1}|| r_{0}\right) C_{L}}=2 * \frac{\omega_{u}}{1+r_{o} / R_{1}}
$$




$$
R_{1}=\frac{r_{0}}{\sqrt{2 r_{o} C_{L} \omega_{u}}-1}
$$

In fact, Equation 4.10 is a rearranged form of Equation 4.5 with $\zeta=1 / \sqrt{2}$ (or 0.707). So once again, considering a real-world application where a device has been characterized with $r_{o}=100 \Omega, f_{u}=1 \mathrm{MHz}$, and $C_{L}=50 \mathrm{nF}$. The same value for $R_{1}$ can be obtained with Equation 4.10.

$$
R_{1}=\frac{100}{\sqrt{2 * 100 * 50 * 10^{-9} * 2 \pi * 10^{6}}-1}=14.4 \Omega
$$

\subsubsection{Practical Implementation Requires an Additional Capacitor}

A shunt resistor by itself is an impractical solution. Because of its small value, a large amount of DC current would be demanded such that the op-amp might shut down or its slewing action would never allow steady state to be reached. A simple way around this is to place a DC-blocking capacitor in series with the compensation resistor. In literature, this is known as an "RC Snubber" that prevents conduction of DC current in addition to acting as energy-absorbing element used to suppress voltage transients (overshoot, in this case). The value of this capacitor should be large relative to the frequencies of operation such that it does not interfere with the resistor's compensating action and can be ignored in the transfer function.

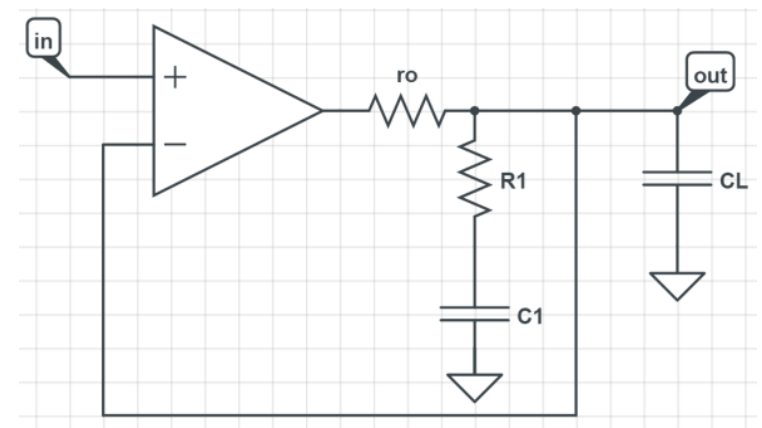

Figure 29. R1 is typically small in value, so $\mathrm{C} 1$ prevents it from conducting a large current.

For this to be an effective strategy, the DC-blocking capacitor should act as a shortcircuit at higher frequencies while absorbing or blocking lower frequencies. Thus, its 
reactance should be small compared to $R_{1}$ at the unity-gain frequency by at least an order of magnitude. An inequality can be expressed under this constraint.

$$
\begin{gathered}
R_{1}>10 *\left|X_{C 1}\right| \text { at } \omega=\frac{\omega_{u}}{1+r_{o} / R_{1}} \\
C_{1}>10 * \frac{1+r_{o} / R_{1}}{R_{1} \omega_{u}}
\end{gathered}
$$

Consider, this time, a compensation simulation with the LM358. Parameters extracted from simulated characterization are $f_{u} \approx 1 \mathrm{MHz}$ and $r_{o} \approx 51 \Omega$ for $C_{L}=$ $50 n F$. Using these numbers values for shunt resistor $R_{1}$ and DC-blocking capacitor $C_{1}$ can be determined using Equations 4.10 and 4.13.

$$
\begin{gathered}
R_{1}=\frac{51}{\sqrt{2 * 51 * 50 * 10^{-9} * 2 \pi * 10^{6}}-1}=10.9 \Omega \\
C_{1}>10 * \frac{1+51 / 10.9}{10.9 * 2 \pi * 10^{6}} \Rightarrow C_{1}>0.83 u F
\end{gathered}
$$

A reasonable standard value of $C_{1}=1 u F$ can be chosen to ensure low reactance at high frequencies. Figure 30 below shows simulations of impulse and frequency response for an LM358 op-amp that is compensated and uncompensated.
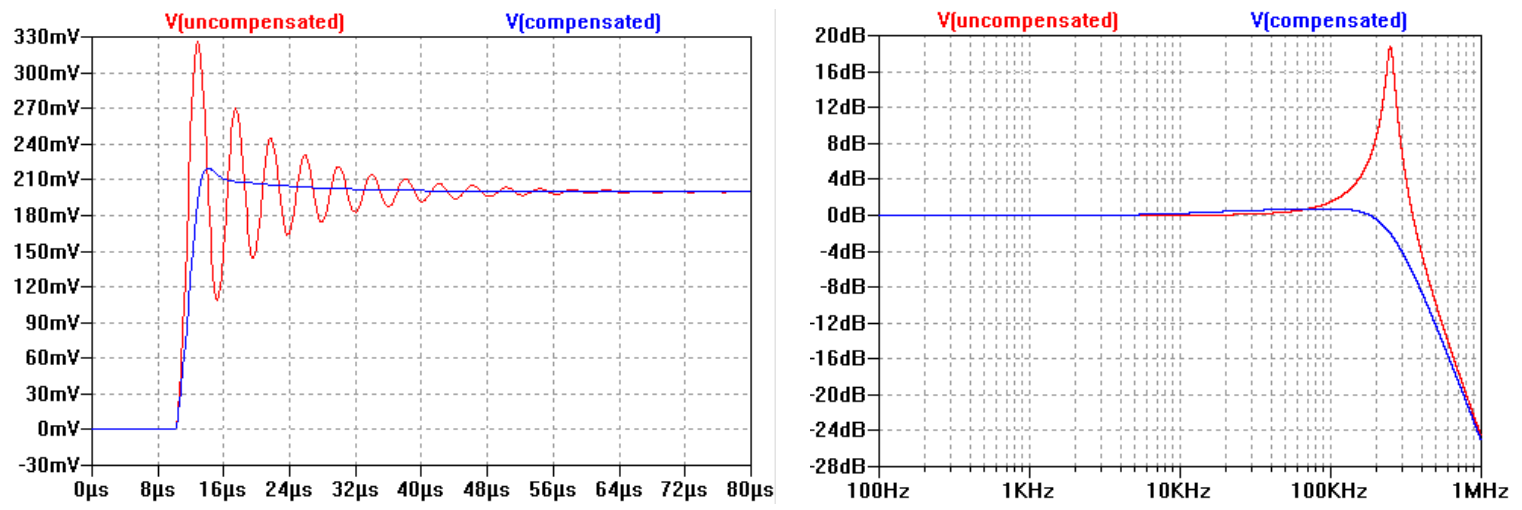

Figure 30. Shunt compensation eliminates ringing and gain peaking.

When uncompensated, there is severe ringing in the time domain that is reflected as a sharp resonance in the frequency domain. When compensated, a small amount of overshoot in time and frequency can also be seen, both of which are most likely artifacts of a Butterworth response with $\zeta=0.707$. 
However, this compensation strategy ends up being expensive because the opamp must drive not only the load, but also the RC snubber network. As simulated in Figure 31, the AC current through the R1-C1 branch is about $26 \mathrm{~dB}(20 \mathrm{x})$ larger than the current through the $50 \mathrm{nF}$ load capacitor. Current conduction has increased by a factor approximately $1+C_{L} / C_{1}$, which is 21 in this case.

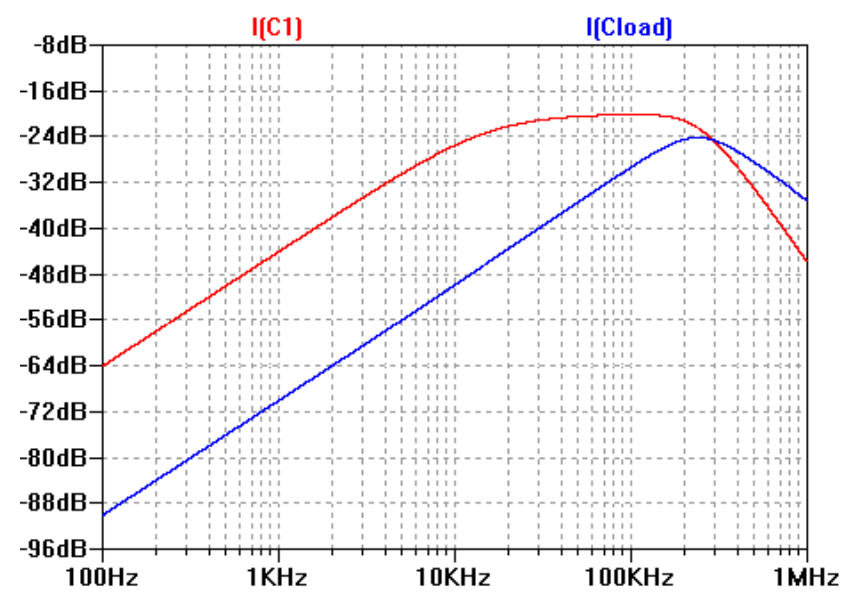

Figure 31. At lower frequencies, $\mathrm{C} 1$ conducts 20 times more current than the load.

\subsection{Method 2: Series Capacitor}

Placing a capacitor in series with the load is a method not found in literature, but the principle behind it is complementary to using a shunt resistor for dealing with the $1 / r_{o} C_{L}$ parasitic pole. Instead of lowering resistance, this circuit attempts to lower the equivalent capacitance, which is realized as the series combination of the compensation capacitor and load capacitor.

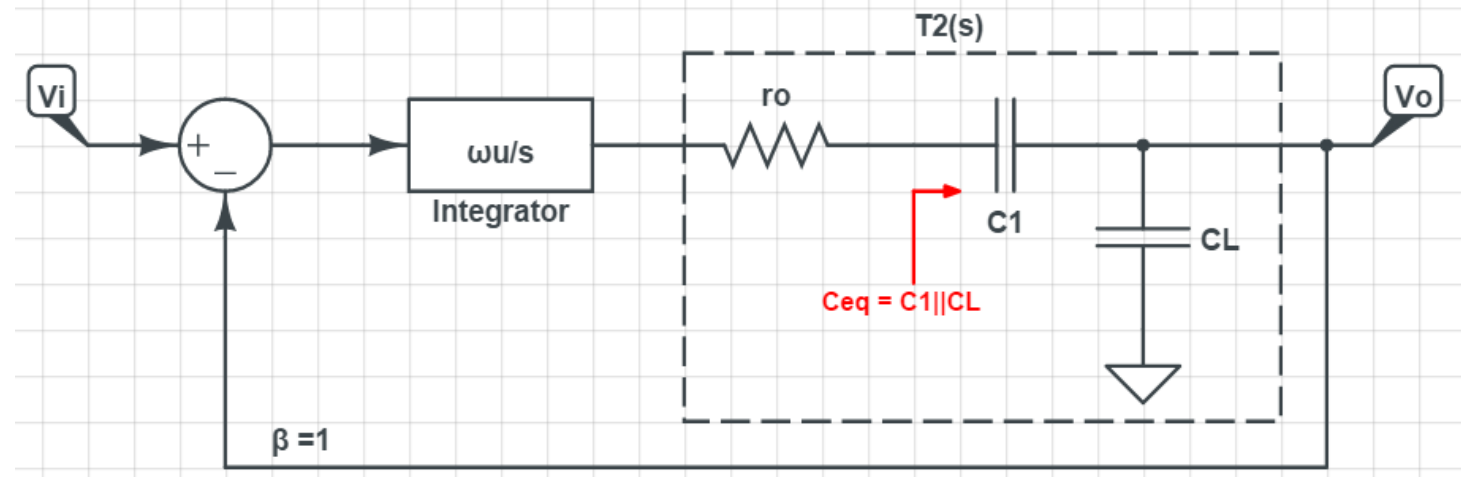

Figure 32. A series capacitor reduces the load capacitance seen. The "|l" operator defines $C_{1} C_{L} /\left(C_{1}+C_{L}\right)$. 
The compensation network $T_{2}$ transfer characteristic is given below. Comparing it with Equation 3.7 (without $C_{1}$ ), it is obvious that $C_{1}$ will influence the closed-loop.

$$
T_{2}(s)=\frac{C_{1}}{r_{0} C_{L} C_{1} s+C_{L}+C_{1}}
$$

\subsubsection{Closed-Loop Analysis with Example Application}

Looking out of the op-amp, the equivalent capacitance seen is $\frac{C_{1} C_{L}}{C_{1}+C_{L}}$, as illustrated in Figure 32. Here, $C_{1}$ and $C_{L}$ forms a voltage divider where feedback is taken which affects loop dynamics. The new characteristic equation from the closed-loop transfer function, rearranged into standard form, is given below.

$$
s^{2}+\frac{\left(C_{L}+C_{1}\right)}{r_{0} C_{1} C_{L}} s+\frac{\omega_{u}}{r_{o} C_{L}}=0
$$

From Equation 4.16 the resemblance between shunt resistor and series capacitor compensation becomes more clear. Poles, natural frequency, and damping ratio are extracted below using this equation.

$$
\begin{gathered}
p_{1}, p_{2}=-\frac{\left(C_{L}+C_{1}\right)}{2 r_{o} C_{1} C_{L}} \pm \sqrt{\frac{\left(C_{L}+C_{1}\right)^{2}}{4 r_{0}^{2} C_{1}^{2} C_{L}^{2}}-\frac{\omega_{u}}{r_{o} C_{L}}} \\
\omega_{n}=\sqrt{\frac{\omega_{u}}{r_{o} C_{L}}} \\
\zeta=\frac{\left(1+\frac{C_{L}}{C_{1}}\right)}{2 \sqrt{r_{o} C_{L} \omega_{u}}}
\end{gathered}
$$

As expected, the additoin of $C_{1}$ has no effect on the loop's natural frequency, and the damping ratio becomes tunable in terms of this capacitor. Obtainig well-behaved feedback is now a matter of selecting a proper value for $C_{1}$.

Consider the same real-world application as before with $r_{o}=100 \Omega, f_{u}=$ $1 \mathrm{MHz}$, and $C_{L}=50 \mathrm{nF}$. From Equation 3.13, it was shown that $\zeta=0.0892$ which is small enough for sustained oscillations in a real circuit. By $\zeta=0.707$ and solving Equation 4.19 for $C_{1}$, the control loop can be tuned for a Butterworth response that dampens out oscillations quickly. 


$$
0.707=\frac{\left(1+\frac{50 * 10^{-9}}{C_{1}}\right)}{2 \sqrt{100 * 50 * 10^{-9} * 2 \pi * 10^{6}}} \Rightarrow C_{1}=7.22 \mathrm{nF}
$$

With this value for $C_{1}$, the capacitive load seen by the op-amp is brought down to a more manageable $C_{e q}=6.3 n F$ so the parasitic pole is shifted back outward by a factor of almost 7 .

\subsubsection{Open-Loop Analysis with Example Application}

Opening the loop, the forward gain can be written using compensation network $T_{2}$ 's transfer funciton.

$$
\begin{aligned}
A_{2}(s) & =\frac{\omega_{u}}{s} * \frac{C_{1}}{r_{0} C_{L} C_{1} s+C_{L}+C_{1}} \\
& =\frac{\omega_{u}}{s} * \frac{C_{1}}{C_{1}+C_{L}} * \frac{1}{r_{o} \frac{C_{1} C_{L}}{C_{1}+C_{L}} s+1}
\end{aligned}
$$

From the open-loop transfer function, it can be seen that introducing $C_{1}$ into the circuit shifts the parasitic (secondary) pole to a higher frequency $\frac{1}{r_{0} \frac{C_{1} C_{L}}{C_{1}+C_{L}}}$ at the cost of reduced gain by a factor of $\frac{C_{1}}{C_{1}+C_{L}}$. If the pole has been shifted far enough, unity-gain will occur at $\frac{\omega_{u}}{1+C_{L} / C_{1}}$ as illustrated in Figure 33 below.

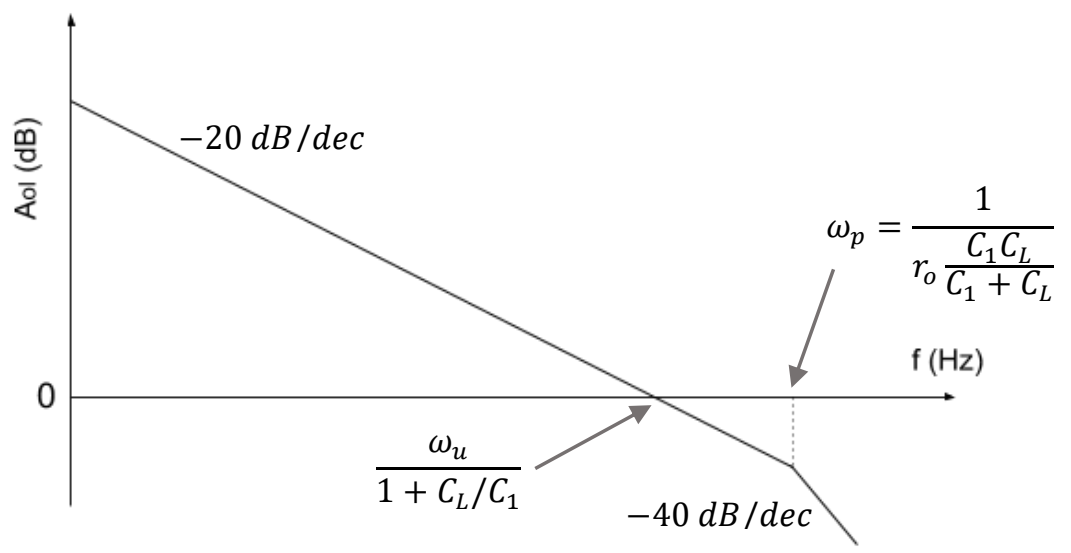

Figure 33. Secondary pole is shifted out, but open-loop gain is reduced. 
With feedback applied, a second-order, type-I (two poles, one integrator) control system si fromed. With one pole at the origin, and as long as the other occurs beyond the unity-gain frequency, phase margin is greater than $45^{\circ}$ and stability is maintained. The preferred location for the second pole is at twice the unity-gain frequency, which results in a Butterworth response. This constraint can be realized by deriving a closed-form expression for $C_{1}$.

$$
\begin{gathered}
\frac{1}{r_{o} \frac{C_{1} C_{L}}{C_{1}+C_{L}}}=2 * \frac{\omega_{u}}{1+C_{L} / C_{1}} \\
C_{1}=\frac{C_{L}}{\sqrt{2 r_{o} C_{L} \omega_{u}}-1}
\end{gathered}
$$

In fact, Equation 4.23 is a rearranged form of Equation 4.19 with $\zeta=1 / \sqrt{2}$ (or 0.707). So once again, considering an application with $r_{o}=100 \Omega, f_{u}=1 \mathrm{MHz}$, and $C_{L}=50 n F$. The same value for $C_{1}$ can be obtained using Equation 4.23.

$$
C_{1}=\frac{50 * 10^{-9}}{\sqrt{2 * 100 * 50 * 10^{-9} * 2 \pi * 10^{6}}-1}=7.22 n F
$$

\subsubsection{Practical Implementation Requires an Additional Resistor}

A series capacitor by itself is an impractical solution because the series connetion of $C_{1}$ and $C_{L}$ causes $V_{o}$ to become an isolated node with no DC path to any other node. It does not have a deterministic initial condition and is not capable driving any feedback. This problem is easily alleviated by placing a resistor in parallel with $C_{1}$ to provide a DC path to and from the $V_{o}$ node. The value of this resistor should be large so it does not interfere with the capacitor's compensating action and can be ignored in the transfer function.

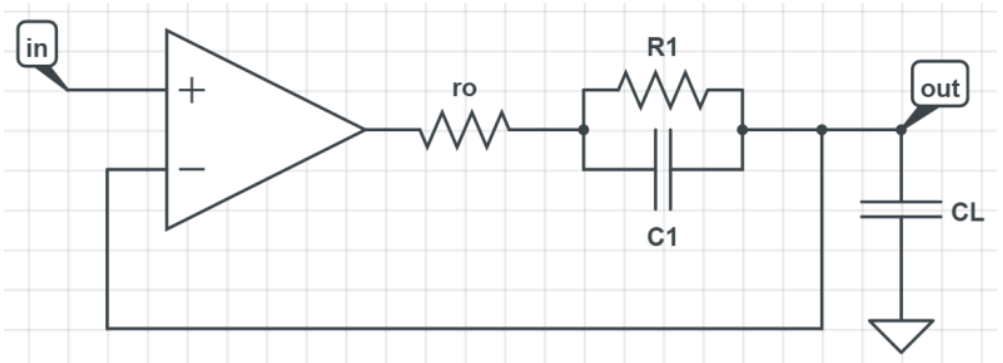

Figure 34. The output node becomes isolated, so an additional R1 is needed to provide a DC path to it. 
For this to be an effective strategy $R_{1}$ should be larger in value than the reactance of $C_{1}$ at the unity-gain frequency by at least an order of magnitude. An inequality can be expressed under this constraint.

$$
\begin{gathered}
R_{1}>10 *\left|X_{C 1}\right| \text { at } \omega=\frac{\omega_{u}}{1+C_{L} / C_{1}} \\
R_{1}>10 * \frac{1+C_{L} / C_{1}}{C_{1} \omega_{u}}
\end{gathered}
$$

Consider again a compensation simulation with the LM358 $f_{u} \approx 1 \mathrm{MHz}$ and $r_{o} \approx$ $51 \Omega$ for $C_{L}=50 \mathrm{nF}$. Using these numbers for series capacitor $C_{1}$ and parallel resistor $R_{1}$ can be determined using Equations 4.23 and 4.26 .

$$
\begin{gathered}
C_{1}=\frac{50 * 10^{-9}}{\sqrt{2 * 51 * 50 * 10^{-9} * 2 \pi * 10^{6}}-1}=10.7 n F \\
R_{1}>10 * \frac{1+(50 / 10.7)}{10.7 * 10^{-9} * 2 \pi * 10^{6}} \Rightarrow C_{1}>844 \Omega
\end{gathered}
$$

A reasonable standard value of $R_{1}=1 \mathrm{k} \Omega$ can be chosen to ensure its resistance is higher than the capacitor's reactance at low frequencies. Figure 35 below shows simulations of impulse and frequency response for an LM358 op-amp that is compensated and uncompensated.
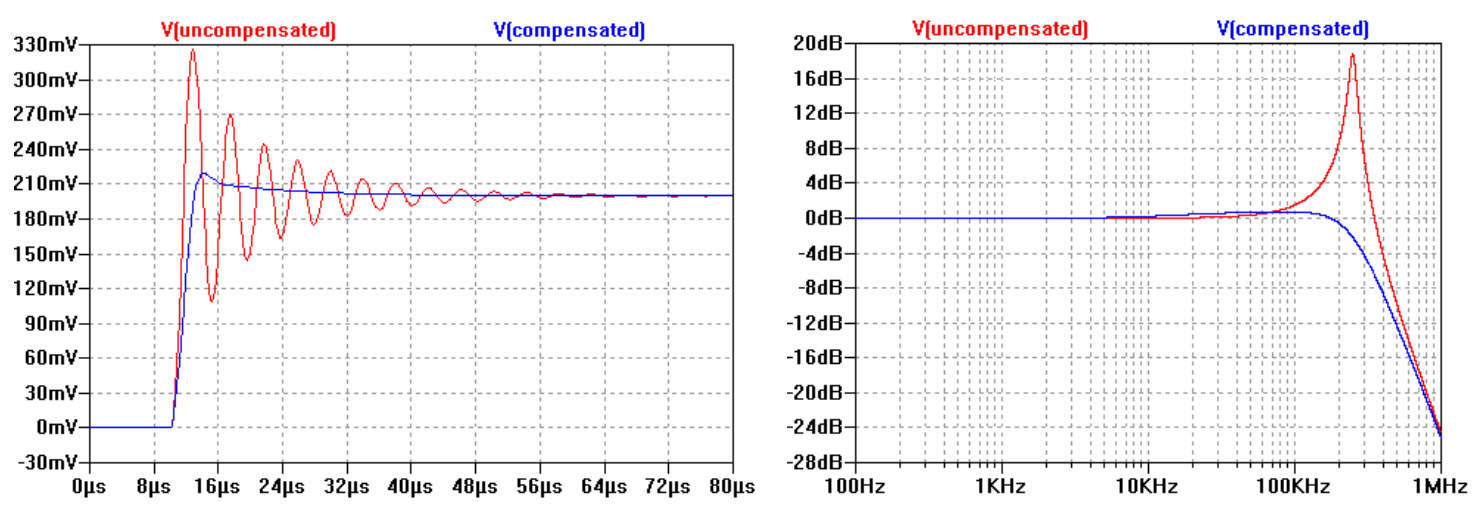

Figure 35. Series compensation eliminates ringing and gain peaking.

When uncompensated, there is severe ringing in the time domain that is reflected as a sharp resonance in the frequency domain. The results are nearly identical to using shunt resistor compensation shown in Figure 30. With series capacitor compensation, a small 
amount of overshoot in time and frequency can be noticed, both of which are likely artifacts of a Butterworth response with $\zeta=0.707$.

However, this compensation strategy too ends up being very expensive because the op-amp needs to be capable of a much larger voltage swing at its output. While voltage across the load follows the input, voltage at the op-amp output is larger by a factor of approximately $1+C_{L} / C_{1}=5.7(\sim 15 \mathrm{~dB})$, as simulated in Figure 36. Clearly, this approach is inappropriate in low voltage supply environments.

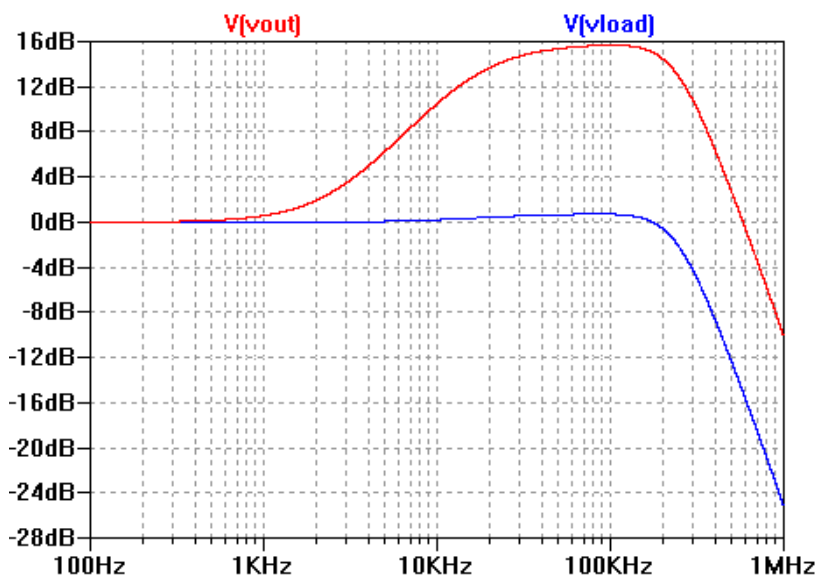

Figure 36. At higher frequencies, the op-amp needs a larger output swing to maintain stable feedback.

\subsection{Proposed Composite Method - Using Both}

Thus far, shunt and series compensation approaches for driving heavy capacitive loads have been presented. This segues into the core purpose of this thesis: a generalized composite compensation strategy that uses both of the aforementioned methods. Since a shunt resistor by itself requires a larger current, and a series capacitor requires a larger voltage swing, a composite technique can be developed using both that makes a better trade-off between current, voltage, and compensation. 


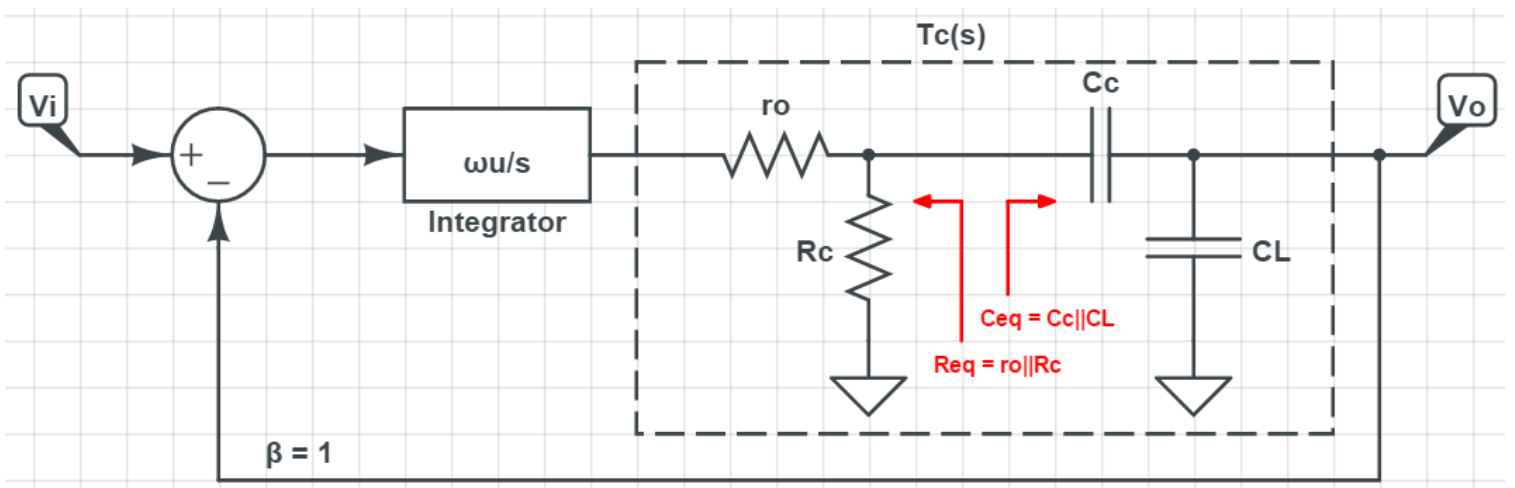

Figure 37. Using shunt and series comepnsation, outptut impedance and load capacitance are reduced.

A complementary $R_{c}-C_{c}$ passive network can be inserted between $r_{o}$ and $C_{L}$ to lower their respective influences on the parasitic pole thereby effectively pushing it outward. Figure 37 illustrates this effect. The compensation network $T_{C}$ transfer function is given below. Compared to an uncompensated loop in $\S 3.2$ and Equation 3.7, it is clear that $R_{C}$ and $C_{c}$ will affect the closed-loop response.

$$
T_{C}(s)=\frac{R_{C} C_{C}}{R_{C} r_{o} C_{C} C_{L} s+\left(R_{C}+r_{o}\right)\left(C_{C}+C_{L}\right)}
$$

\subsubsection{Closed-Loop Analysis with Example Application}

Looking into the op-amp, the equivalent output resistance has been reduced to $r_{o} \| R_{C}$, and looking out of the op-amp the equivalent capacitive load seen is $\frac{C_{1} C_{L}}{C_{1}+C_{L}}$. With this passive network added in, the new characteristic equation from the closed-loop transfer function, rearranged into standard form, is given below.

$$
s^{2}+\frac{\left(r_{o}+R_{C}\right)\left(C_{L}+C_{C}\right)}{R_{C} r_{0} C_{C} C_{L}} s+\frac{\omega_{u}}{r_{o} C_{L}}=0
$$

From this equation, poles, natural frequency, and damping ratio are extracted.

$$
\begin{gathered}
p_{1}, p_{2}=-\frac{\left(r_{o}+R_{C}\right)\left(C_{L}+C_{C}\right)}{2 R_{C} r_{o} C_{C} C_{L}} \pm \sqrt{\frac{\left(r_{o}+R_{C}\right)^{2}\left(C_{L}+C_{C}\right)^{2}}{4 R_{C}^{2} r_{0}^{2} C_{C}^{2} C_{L}^{2}}-\frac{\omega_{u}}{r_{o} C_{L}}} \\
\omega_{n}=\sqrt{\frac{\omega_{u}}{r_{o} C_{L}}}
\end{gathered}
$$




$$
\zeta=\frac{\left(1+\frac{C_{L}}{C_{C}}\right)\left(1+\frac{r_{o}}{R_{C}}\right)}{2 \sqrt{C_{L} r_{o} \omega_{u}}}
$$

The natural frequency does not change as expected, but the damping ratio now contains two unknown variables: $R_{c}$ and $C_{c}$. Obtaining a well-behaved feedback now becomes an optimization problem based on the given application.

To this end, new parameters can be described as the "compensation coefficient" for $r_{o} / R_{C}$ and $C_{L} / C_{C}$.

$$
\begin{gathered}
\eta_{R}=1+\frac{r_{o}}{R_{C}} \\
\eta_{C}=1+\frac{C_{L}}{C_{C}}
\end{gathered}
$$

Following the discussions in $\S 4.1 .3$ and $\S 4.2 .3, \eta_{R}$ will dictates how much more current the op-amp must supply, and $\eta_{C}$ dictates how much more voltage swing it must have available. Given the recent trend in electronics toward lower supply voltages, the following example will optimize $\zeta$ for a low voltage supply environment. This requires $\eta_{R}$ to carry a greater weight than $\eta_{C}$. For the sake of argument, $\eta_{R}=2 * \eta_{C}$ is set arbitrarily and substituted in which simplifies the damping ratio formula.

$$
\zeta=\frac{\left(\eta_{C}\right)\left(2 \eta_{C}\right)}{2 \sqrt{C_{L} r_{o} \omega_{u}}}=\frac{2 \eta_{C}^{2}}{2 \sqrt{C_{L} r_{o} \omega_{u}}}
$$

Once $\eta_{C}$ is calculated using this formula (for a desired $\zeta$ ), $\eta_{R}, R_{C}$, and $C_{C}$ can be computed.

Again, consider a situation with the LM358, $f_{u} \approx 1 \mathrm{MHz}$ and $r_{o} \approx 51 \Omega$ for $C_{L}=$ $47 n F$ this time with a desired $\zeta=0.707$.

$$
0.707=\frac{2 \eta_{C}^{2}}{2 \sqrt{51 * 47 * 10^{-9} * 2 \pi * 10^{6}}} \Rightarrow \eta_{C}=1.66
$$

This compensation coefficient dictates that $C_{C}=71 n F$ and $R_{C}=22 \Omega$.

\subsubsection{Open-Loop Analysis with Example Application}

Opening the loop, the forward gain can be calculated as the product of the integrator and $T_{C}(s)$.

$$
A_{C}(s)=\frac{\omega_{u}}{s} * \frac{R_{C} C_{C}}{R_{C} r_{o} C_{C} C_{L} s+\left(R_{C}+r_{o}\right)\left(C_{C}+C_{L}\right)}
$$




$$
=\frac{\omega_{u}}{s} * \frac{R_{C} C_{C}}{\left(r_{o}+R_{C}\right)\left(C_{L}+C_{C}\right)} * \frac{1}{\frac{R_{C} r_{o}}{R_{C}+r_{o}} \frac{C_{C} C_{L}}{C_{C}+C_{L}} s+1}
$$

From the open-loop transfer function, it can be seen that introducing $R_{C}$ and $C_{C}$ shifts the parasitic pole to a higher frequency $\omega_{p}=\frac{1}{\frac{R_{C} r_{o}}{R_{C}+r_{o}} \frac{C_{C} C_{L}}{C_{C}+C_{L}}}$ at the cost of reduced gain by a factor of $\frac{R_{C} C_{C}}{\left(r_{o}+R_{C}\right)\left(C_{L}+C_{C}\right)}$. This also pulls in the unity gain frequency to $\frac{\omega_{u}}{\left(1+\frac{C_{L}}{C_{C}}\right)\left(1+\frac{r_{o}}{R_{C}}\right)}$, as illustrated in Figure 38.

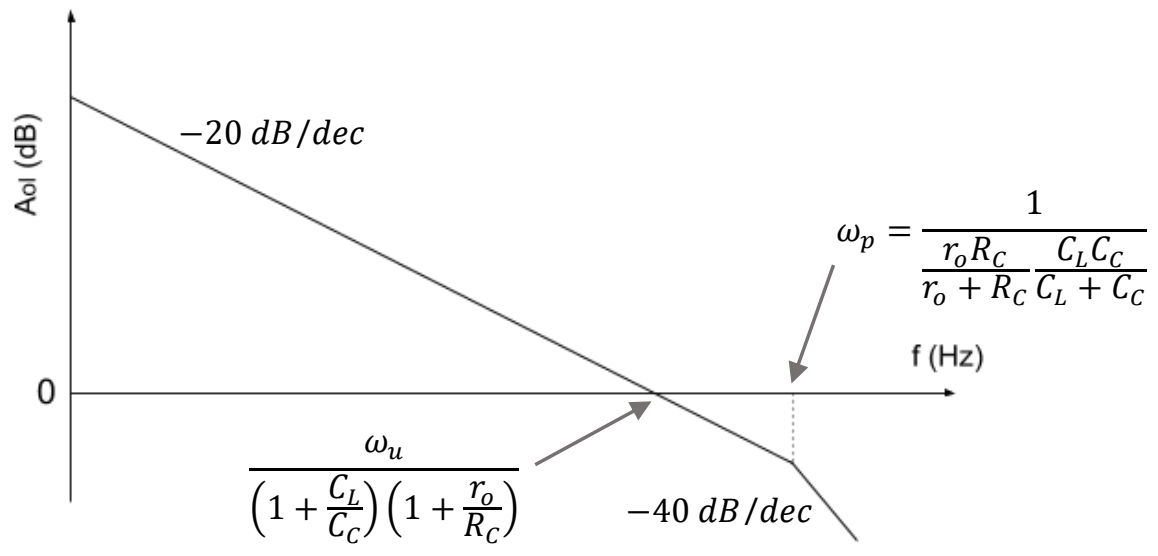

Figure 38. Secondary pole is shifted out, but open-loop gain is reduced.

When feedback is applied, the system remains second-order, type-I as before. Stability is maintained as long as the second pole is far enough to allow at least $45^{\circ}$ of phase margin. For a Butterworth response, the preferred location is at twice the unity-gain frequency. This constraint is realized by writing a closed-form equation

$$
\frac{1}{\frac{R_{C} r_{o}}{R_{C}+r_{o}} \frac{C_{C} C_{L}}{C_{C}+C_{L}}}=2 * \frac{\omega_{u}}{\left(1+\frac{C_{L}}{C_{C}}\right)\left(1+\frac{r_{o}}{R_{C}}\right)}
$$

It turns out that this equation can be rearranged to take the same form as Equation 4.32 with $\zeta=0.707$. If $\eta_{R}$ and $\eta_{C}$ are defined similarly and shunt compensation is set to carry a greater weight than series $\left(\eta_{R}=2 * \eta_{C}\right)$, then Equation 4.38 can be used to calculate the same values for $C_{C}=71 \mathrm{nF}$ and $R_{C}=22 \Omega$. 


\subsubsection{Practical Implementation Requires Two Components}

Frequency compensating the op-amp using just $R_{C}-C_{C}$ has the same issues as using either series or shunt compensation by itself. $R_{C}$ draw a large DC current and $C_{C}$ would create an isolated feedback (output) node with no DC path. To mitigate these obstacles, $R_{1}$ and $C_{1}$ must be added as shown in Figure 39. $C_{1}$ forms a snubber network with $R_{C}$ and will prevent conduction of a large DC current, and $R_{1}$ provides the feedback (output) node with a DC path so it can be properly driven.

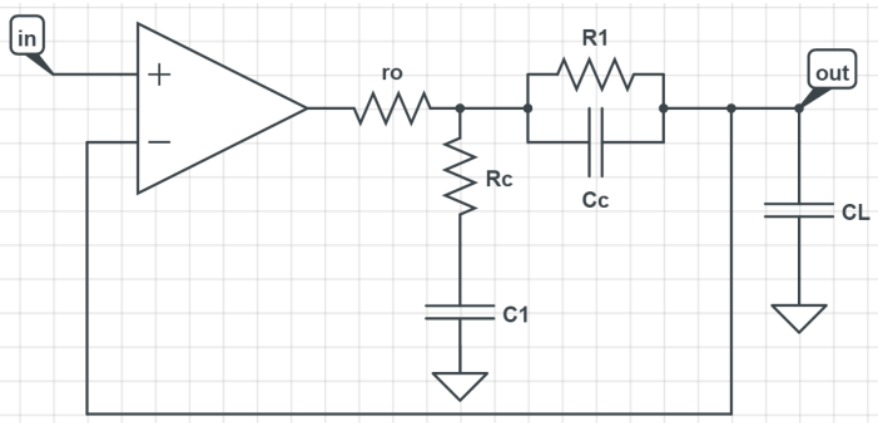

Figure 39. Additional DC blocking capacitor and DC conducting resistor are required.

For this to be an effective strategy $C_{1}$ should present lower impedance than $R_{C}$ at high frequency, while $R_{1}$ should present higher impedance than $C_{C}$ at high frequency. To put "high frequency" in a relative context, impedances should be respectively higher or lower by an order of magnitude at the new unity-gain frequency.

$$
\begin{aligned}
& 10 *\left|X_{C 1}\right|<R_{C} \\
& R_{1}>10 *\left|X_{C_{C}}\right|
\end{aligned}
$$

Solving these inequalities for the additional $C_{1}$ and $R_{1}$

$$
\begin{aligned}
& C_{1}>10 * \frac{\left(1+\frac{C_{L}}{C_{C}}\right)\left(1+\frac{r_{o}}{R_{C}}\right)}{R_{C} \omega_{u}}=10 * \frac{\left(\eta_{C}\right)\left(\eta_{R}\right)}{R_{C} \omega_{u}} \\
& R_{1}>10 * \frac{\left(1+\frac{C_{L}}{C_{C}}\right)\left(1+\frac{r_{o}}{R_{C}}\right)}{C_{C} \omega_{u}}=10 * \frac{\left(\eta_{C}\right)\left(\eta_{R}\right)}{C_{C} \omega_{u}}
\end{aligned}
$$

Following the example with $f_{u} \approx 1 \mathrm{MHz}$ and $r_{o} \approx 51 \Omega$ for $C_{L}=47 \mathrm{nF}$, it was found that $C_{C}=71 \mathrm{nF}$ and $R_{C}=22 \Omega$. These numbers yield $C_{1}>399 \mathrm{nF}$ and $R_{1}>$ $124 \Omega$. These can be rounded up to standard values of $470 \mathrm{nF}$ and $150 \Omega$. Figure 40 
shows simulations of impulse and frequency response for an LM358 op-amp with this compensation and one without compensation.
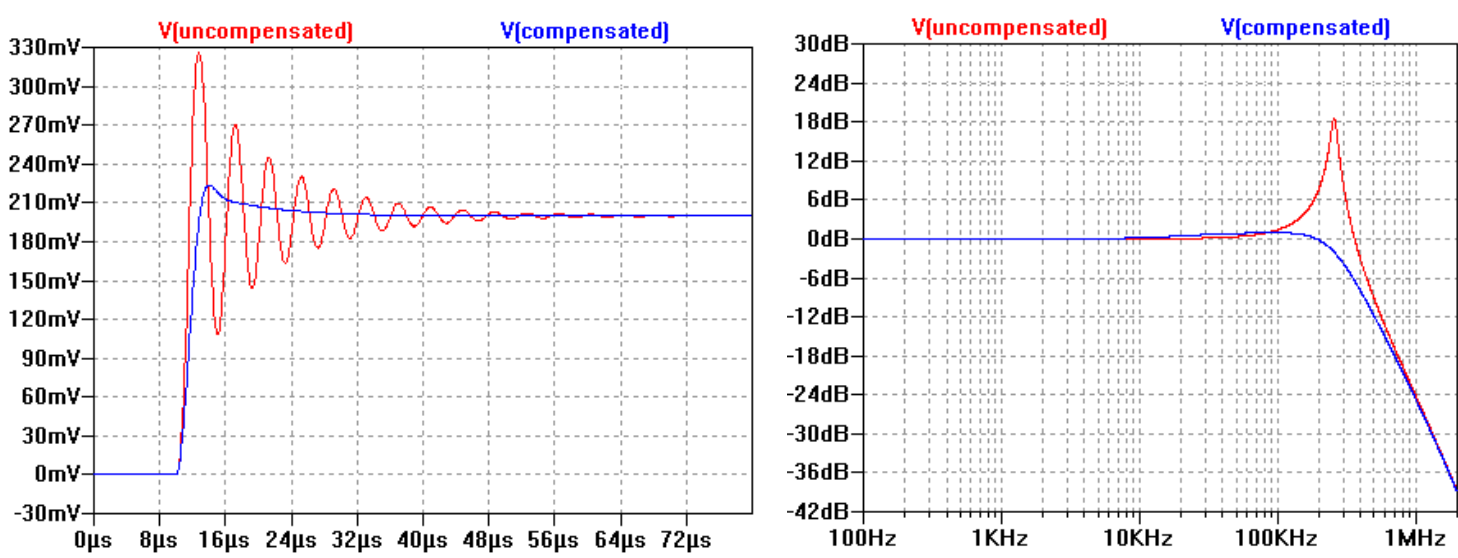

Figure 40. Composit compensation eliminates ringing and gain peaking.

When compensated, a small amount of overshoot in time and frequency can be notice, but they are likely artifacts of a Butterworth response. Series damping coefficient $\eta_{C}=$ $1+\frac{C_{L}}{C_{C}}=1.66(4.4 \mathrm{~dB})$ can also be verified. Figure 41 illustrates that at higher frequencies the voltage at the output of the op-amp is approximately $4.35 \mathrm{~dB}$ greater, which is very close to what was expected.
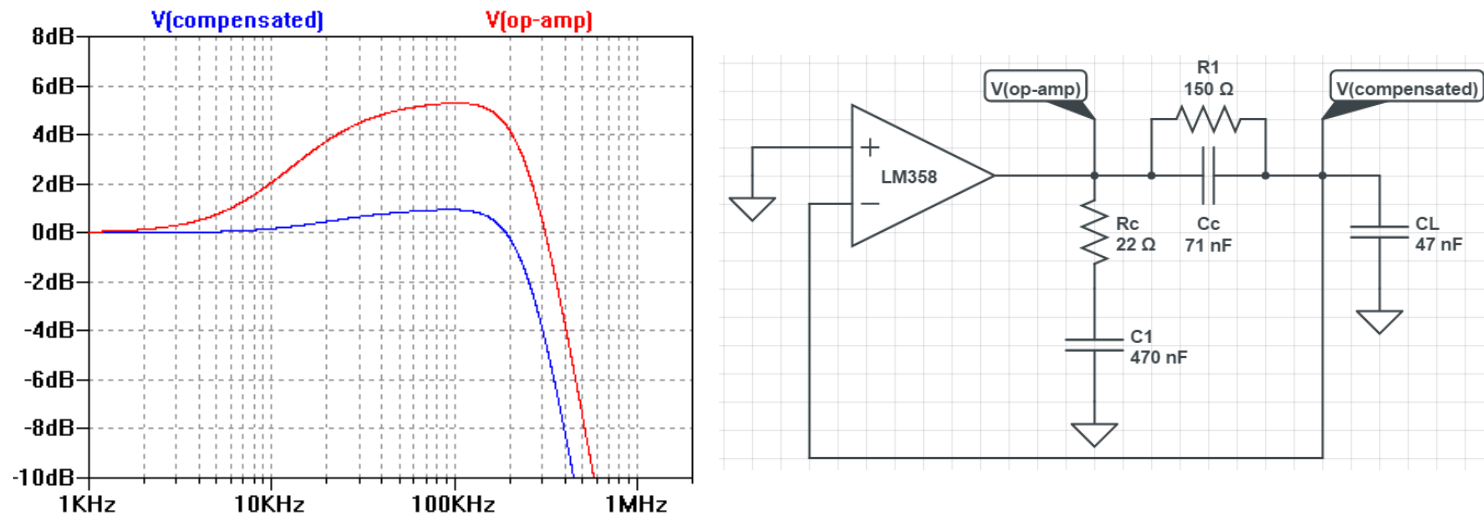

Figure 41. At higher frequencie, the op-amp must swing $4.35 \mathrm{~dB}$ more to maintain unity feedback.

It has been shown that a composite technique with series and shunt compensation elements exists and can be used successfully. The following chapter will outline a step by step guide to using this technique and how it can be optimized for various applications. 


\section{Chapter 5: Practical Design of the Composite Compensation Network}

Now that sufficient background information, modeling, and simulations have been considered in detail, a design methodology can be outlined. For a known load, the general process is:

1. Characterize the op-amp's actual GBW $\left(\omega_{u}\right)$ using a non-inverting configuration.

2. Characterize the op-amp's perceived $r_{0}$ using a follower configuration driving $C_{L}$.

3. Solve for the apparent damping ratio $\zeta$ and establish a desired $\zeta$.

4. Consider operating environment and set relationship between compensation coefficients $\eta_{C}$ and $\eta_{R}$.

5. Solve for required shunt resistor, $R_{C}$, and series capacitor $C_{C}$.

6. Determine values for DC blocking capacitor, $C_{1}$, and DC conducting resistor $R_{1}$.

7. Simulate, optimize, prototype.

The following sections summarize the Composite Compensation Technique developed in this thesis. Finer points that the designer might wish to consider are highlighted. Figures and equations are repeated here for convenience.

\subsection{Determine the Actual Device Characteristics}

The datasheet might say one thing, but more likely than not devices perform better than their spec. In some rare cases they perform poorer than expected which is why it's important to quantify the performance of the device at hand.

\subsubsection{Gain Bandwidth $\left(G B W, \omega_{u}\right)$}

It may still be reasonable to just use the given, nominal GBW, but it can be easily determined using an inverting amplifier configuration. Sweeping the input up to a

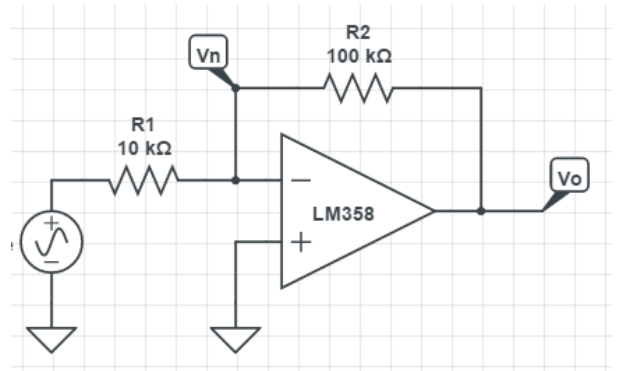
frequency where $V n$ has minimal distortion, $V o$ and $V n$ should be measured at a handful of points. GBW $\left(\omega_{u}\right)$ can be determined using the formula below.

$$
A_{o l}=\frac{V o}{V n} \Rightarrow f_{G B W}=f_{\text {in }} * A_{o l}
$$

Averaging the GBW should be a sufficient measure of its approximate value. 


\subsubsection{Output Impedance $\left(r_{o}\right)$}

The output impedance of an op-amp depends virtually on every environmental and circuit parameter, the most important ones being DC output current, supply voltage, loading, and device PSRR. So, when characterizing $r_{0}$ it is important to replicate supply voltage and load that will finally be used. The op-amp should be placed in a voltage follower (buffer) configuration driving the capacitive load (dual split supply is assumed, but in single supply operation, the positive terminal can be tied to $V_{D D} / 2$ ). The oscillating waveform of $V_{\text {out }}$ should be viewed on an oscilloscope so its approximate frequency

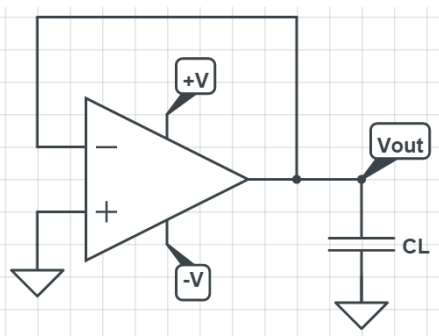

$\left(f_{\text {osc }}\right)$ can be noted. The following formula can be used to determine the output impedance.

$$
r_{o}=\frac{f_{G B W}}{2 \pi * C_{L} * f_{o s c}^{2}}
$$

The experimentally determined $f_{G B W}$ can be used.

On a side note, it is possible that the amplifier is well-designed and can maintain a DC output on a highly capacitive load. Its step response, however, will almost certainly exhibit ringing, in which case the ringing frequency can be used instead of $f_{o s c}$.

\subsection{Determine Desired Damping Ratio (ろ)}

Sustained oscillations are indicative of an exceedingly underdamped system which can be quantified using $\zeta=\frac{1}{2 \sqrt{r_{o} C_{L} \omega_{u}}}$. Knowing this value may give insight into how much compensation is required. Typically, a closed-loop with $\zeta<0.2$ will ring indefinitely. If $0.2<\zeta<0.8$, ringing may require a relatively long time to die out. Although $\zeta=0.707$ represents a Butterworth response with widest and flattest possible passband, it may not be realizable due to nonlinearity in a real circuit. Finally, $\zeta=1$ represents a critically damped response with the quickest convergence to an impulse in the time domain, but damping upwards of $\zeta=2$ may be required.

At this point it is up to the designer to choose a value for $\zeta$. The range of $0.707 \leq \zeta \leq 2$ is desirable for a well-behaved system. In general, less compensation is required for a lower value which translates into lower power and greater available voltage swing. 


\subsection{Consider Compensation Type for Application}

The composite method relies on two modes of frequency compensation that aid in shifting the parasitic pole outward. Using a shunt resistor to lower output impedance but increases current consumption, while a series capacitor lowers load capacitance but reduces output voltage swing.

\subsubsection{Relate $\eta_{C}$ and $\eta_{R}$, Calculate $C_{C}$ and $R_{C}$}

To quantify these effects, two additional parameters are defined that appear in the compensated damping ratio equation.

$$
\zeta=\frac{\left(\eta_{C}\right)\left(\eta_{R}\right)}{2 \sqrt{C_{L} r_{o} \omega_{u}}}
$$

$\eta_{C}=1+\frac{C_{L}}{C_{C}}$ and $\eta_{R}=1+\frac{r_{o}}{R_{C}}$ are referred to as series and shunt damping coefficients that reveal how much more current and voltage is required due to the respective elements. More importantly though one can be defined in terms of the other so priority can be assigned.

For example, the designer might decide that maintaining voltage swing at the output is a more important tradeoff than current so $\eta_{R}=2 \eta_{C}$ can be assigned. Substituting this in, the damping ratio equation can be simplified to having only one unknown variable.

$$
\zeta=\frac{2 \eta_{C}^{2}}{2 \sqrt{C_{L} r_{o} \omega_{u}}}
$$

With the desired $\zeta$ known, $\eta_{C}$ can be solved for which allows a solution for $\eta_{R}, C_{C}$, and $R_{C}$. However, the theoretical values for these components may not actually correspond with standard valued parts. For example, if $C_{C}=71 n F$ was calculated, then the nearest available standard value of $68 n F$ will have to be chosen to keep costs low. But to allay any concerns, the corrective property of this technique is relatively insensitive to component tolerances. 


\subsubsection{Determine Values for DC-Blocking $C_{1}$ and DC-Conducting $R_{1}$}

Lastly, a DC blocking capacitor is required to prevent $R_{C}$ from conducting too much DC current, and a parallel resistor is needed to provide the output with a DC path. However, these additional components should not affect the transfer characteristic of $C_{C}$ and $R_{C}$ that enables frequency compensation. So as the unity-gain frequency approaches, $C_{1}$ 's impedance should reduce to an order of magnitude less than $R_{C}$ 's impedance and $R_{1}$ 's impedance be an order of magnitude greater than $C_{C}$. The following formulas can be

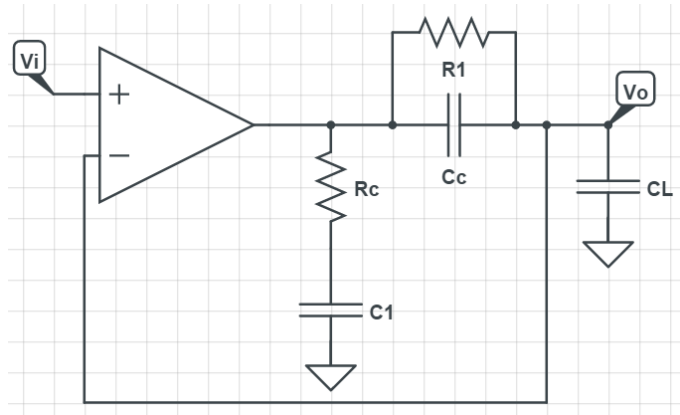
used to realize this.

$$
\begin{aligned}
& C_{1}>10 * \frac{\left(\eta_{C}\right)\left(\eta_{R}\right)}{R_{C} \omega_{u}} \\
& R_{1}>10 * \frac{\left(\eta_{C}\right)\left(\eta_{R}\right)}{C_{C} \omega_{u}}
\end{aligned}
$$

Once again standard values can be used, but should be no larger than needed. A larger $R_{1}$ will increase loading with the circuit that $V_{o}$ is driving, and a larger $C_{1}$ will increase $\mathrm{AC}$ current through the shunt branch (which draws most of the op-amp output current).

\subsection{Simulate, Tweak, and Prototype}

Finally, the compensation network design is complete and should be simulated to verify its utility. Figures of merit include the cutoff frequency and gain-peaking suppression in the frequency domain, impulse overshoot and available voltage swing in the time domain. However, simulations may not be entirely reliable. The designer is encouraged to evaluate the real circuit with different numbers for damping ratio and compensation coefficients. 


\section{Chapter 6: Lab Experiment with Compensated Op-Amps}

\subsection{LM358 Composite Compensation}

Recalling characterization data from $\$ 2.3 .1$, values for $C_{C}$ and $R_{C}$ are calculated under the constraints $\zeta=1$ and $\eta_{R}=2 \eta_{C}$.

\begin{tabular}{|c|ccc|cc|}
\hline Load & \multicolumn{3}{|c|}{ Observed Instability } & \multicolumn{2}{c|}{ Compensation Components } \\
\hline CL (nF) & $\mathbf{f , o s c}(\mathbf{k H z})$ & $\mathbf{f , p o l e ~}(\mathbf{k H z})$ & $\mathbf{r}_{\mathbf{o}}(\mathbf{\Omega})$ & $\mathbf{C c}(\mathbf{n F})$ & $\mathbf{R c}(\mathbf{\Omega})$ \\
\hline 1 & 450 & 171.6 & 927 & 1.6 & 414 \\
\hline 4.7 & 120 & 12.2 & 2775 & 2.2 & 526 \\
\hline 10 & 100 & 8.5 & 1878 & 4.1 & 320 \\
\hline 47 & 79.1 & 5.3 & 639 & 16.4 & 95 \\
\hline 100 & 67.7 & 3.9 & 410 & 31.5 & 56 \\
\hline
\end{tabular}

Table 8. Compensation components calculated for a critically damped response.

With precise values calculated, standard components close in value must be selected based on availability. The additional required $R_{1}$ and $C_{1}$ are calculated and selected as well, and the resulting damping and compensation coefficients are determined.

\begin{tabular}{|c|cc|cc|ccc|}
\hline Load & \multicolumn{2}{|c|}{ Compensation Components } & \multicolumn{4}{|c|}{ Additional Components } & \multicolumn{3}{|c|}{ Stability and Coefficients } \\
\hline CL $(\mathbf{n F})$ & $\mathbf{C c}(\mathbf{n F})$ & $\mathbf{R c}(\boldsymbol{\Omega})$ & $\mathbf{R 1}(\boldsymbol{\Omega})$ & $\mathbf{C 1}(\mathbf{n F})$ & Damping $(\boldsymbol{\zeta})$ & $\boldsymbol{\eta}_{\boldsymbol{R}}$ & $\boldsymbol{\eta}_{\boldsymbol{C}}$ \\
\hline 1 & 1.5 & 390 & $5.1 \mathrm{k}$ & 22 & 1.07 & 3.4 & 1.7 \\
\hline 4.7 & 2.2 & 470 & $15 \mathrm{k}$ & 68 & 1.10 & 6.9 & 3.1 \\
\hline 10 & 3.3 & 330 & $12 \mathrm{k}$ & 100 & 1.14 & 6.7 & 4.0 \\
\hline 47 & 15 & 91 & $3 \mathrm{k}$ & 680 & 1.11 & 8.0 & 4.1 \\
\hline 100 & 33 & 51 & $1.5 \mathrm{k}$ & 1000 & 1.04 & 9.0 & 4.0 \\
\hline
\end{tabular}

Table 9. Resistors and capacitors rounded to standard values so damping ratio stays above 1. 


\section{LM358 Compensated Impulse Response}
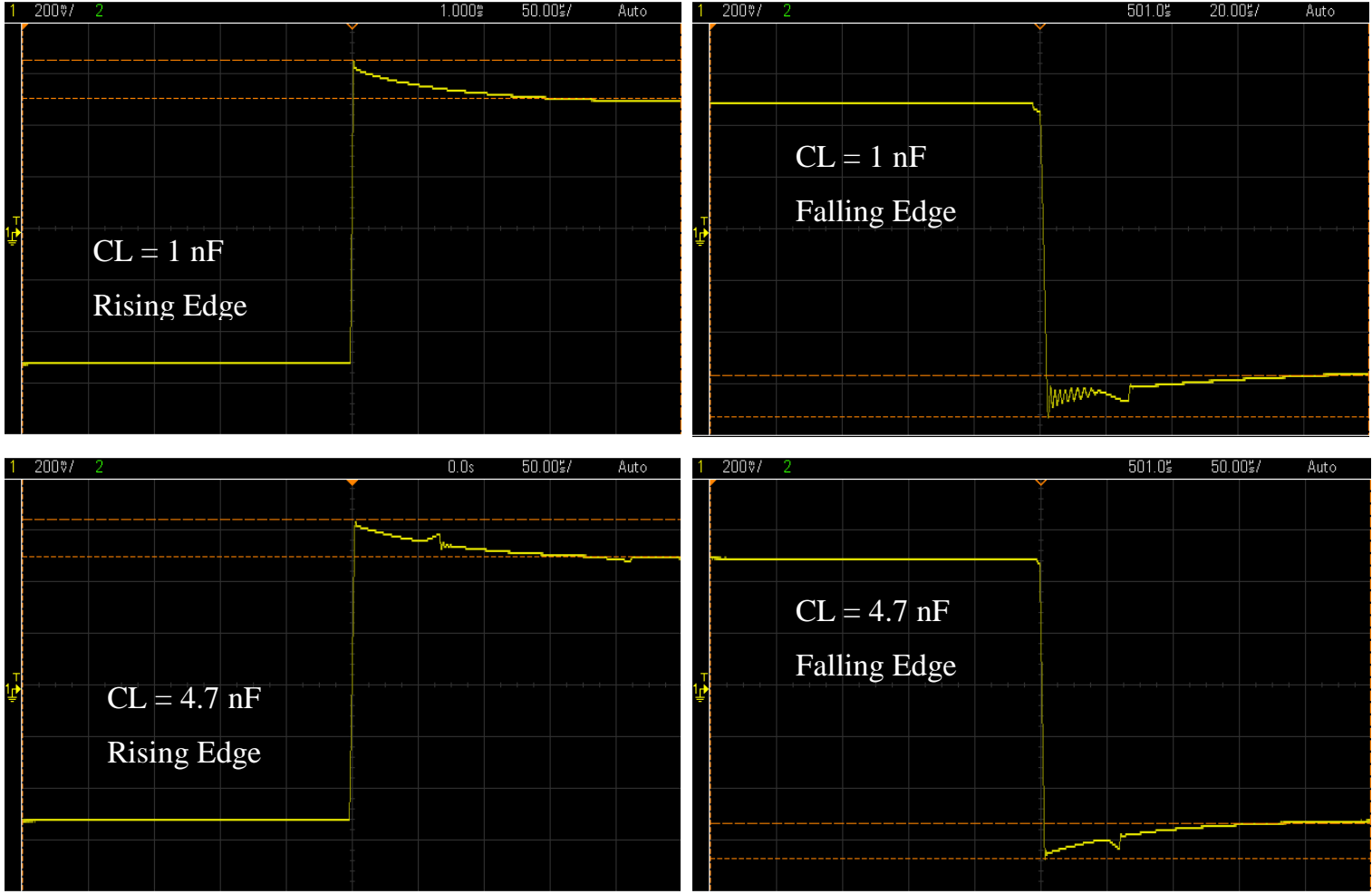

Figure 42. Natural frequency is damped out when there is no input. About $15 \%$ overshoot is present with a $1 \mathrm{~V}$ impulse, and some ringing is also appears in the falling edge of the $1 \mathrm{nF}$ load. This is likely due to the non-symmetric design of the output stage.

\section{LM358 Compensated Linear Signal Response}
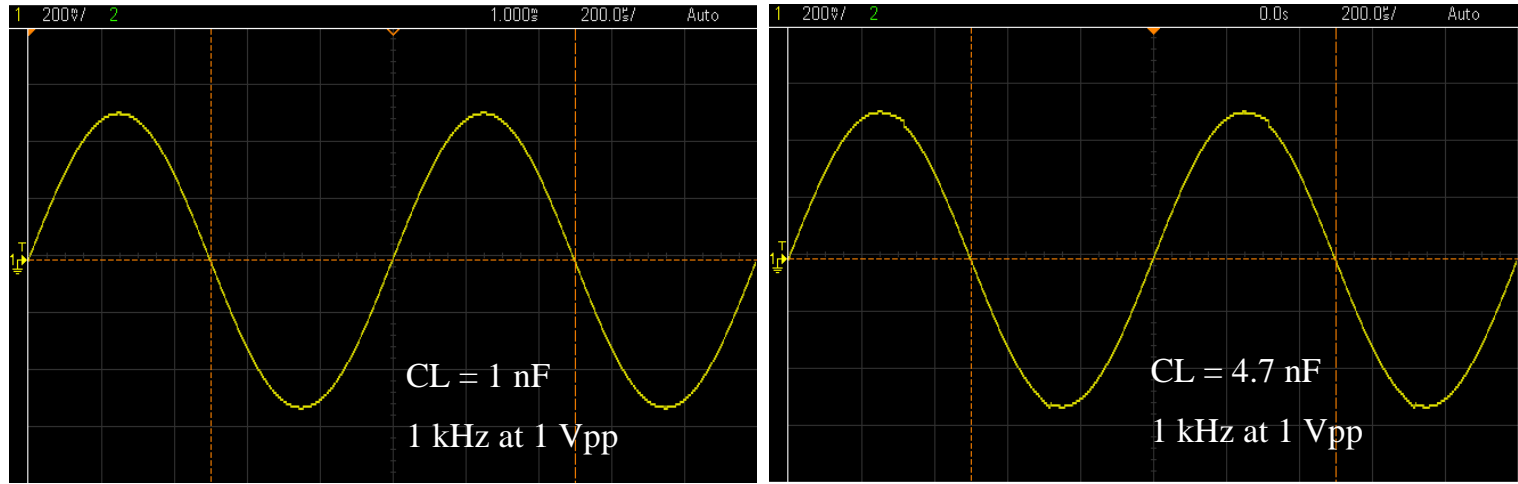

Figure $43.1 \mathrm{nF}$ is driven without any noticable distrotion. For the larger load, $4.7 \mathrm{nF}$, some evidence of distortion is apparent because of output stage deadzones creeping in along the peaks and troughs. 


\subsection{LMC662 Composite Compensation}

Recalling characterization data from $\$ 2.3 .2$, values for $C_{C}$ and $R_{C}$ are calculated under the constraints $\zeta=1$ and $\eta_{R}=2 \eta_{C}$.

\begin{tabular}{|c|ccc|cc|}
\hline Load & \multicolumn{3}{|c|}{ Observed Instability } & \multicolumn{2}{c|}{ Compensation Components } \\
\hline CL $(\mathbf{n F})$ & $\mathbf{f , o s c}(\mathbf{k H z})$ & $\mathbf{f , p o l e}(\mathbf{k H z})$ & $\mathbf{r}_{\mathbf{o}}(\mathbf{\Omega})$ & $\mathbf{C c}(\mathbf{n F})$ & $\mathbf{R c}(\mathbf{\Omega})$ \\
\hline 1 & 570 & 181.5 & 877 & 1.3 & 345 \\
\hline 4.7 & 286 & 45.7 & 741 & 3.1 & 185 \\
\hline 10 & 217 & 26.3 & 605 & 5.3 & 128 \\
\hline 47 & 157 & 13.8 & 246 & 19.8 & 43 \\
\hline 100 & 129 & 9.3 & 171 & 36.7 & 27 \\
\hline
\end{tabular}

Table 10. Compensation components calculated for a critically damped response.

With precise values calculated, standard components close in value must be selected based on availability. The additional required $R_{1}$ and $C_{1}$ are calculated and selected as well, and the resulting damping and compensation coefficients are determined.

\begin{tabular}{|c|cc|cc|ccc|}
\hline Load & \multicolumn{2}{|c|}{ Compensation Components } & \multicolumn{4}{|c|}{ Additional Components } & \multicolumn{3}{|c|}{ Stability and Coefficients } \\
\hline $\mathbf{C L}(\mathbf{n F})$ & $\mathbf{C c}(\mathbf{n F})$ & $\mathbf{R c}(\boldsymbol{\Omega})$ & $\mathbf{R 1}(\boldsymbol{\Omega})$ & $\mathbf{C 1}(\mathbf{n F})$ & Damping $(\boldsymbol{\zeta})$ & $\boldsymbol{\eta}_{\boldsymbol{R}}$ & $\boldsymbol{\eta}_{\boldsymbol{C}}$ \\
\hline 1 & 1 & 330 & $6.8 \mathrm{k}$ & 22 & 1.16 & 3.7 & 2.0 \\
\hline 4.7 & 2.2 & 200 & $6.2 \mathrm{k}$ & 68 & 1.18 & 4.7 & 3.1 \\
\hline 10 & 4.7 & 120 & $3.6 \mathrm{k}$ & 220 & 1.15 & 6.0 & 3.1 \\
\hline 47 & 15 & 47 & $1.6 \mathrm{k}$ & 680 & 1.13 & 6.2 & 4.1 \\
\hline 100 & 33 & 27 & 820 & 1000 & 1.07 & 7.3 & 4.0 \\
\hline
\end{tabular}

Table 11. Resistors and capacitors rounded to standard values so damping ratio stays above 1. 


\section{LMC662 Compensated Impulse Response}
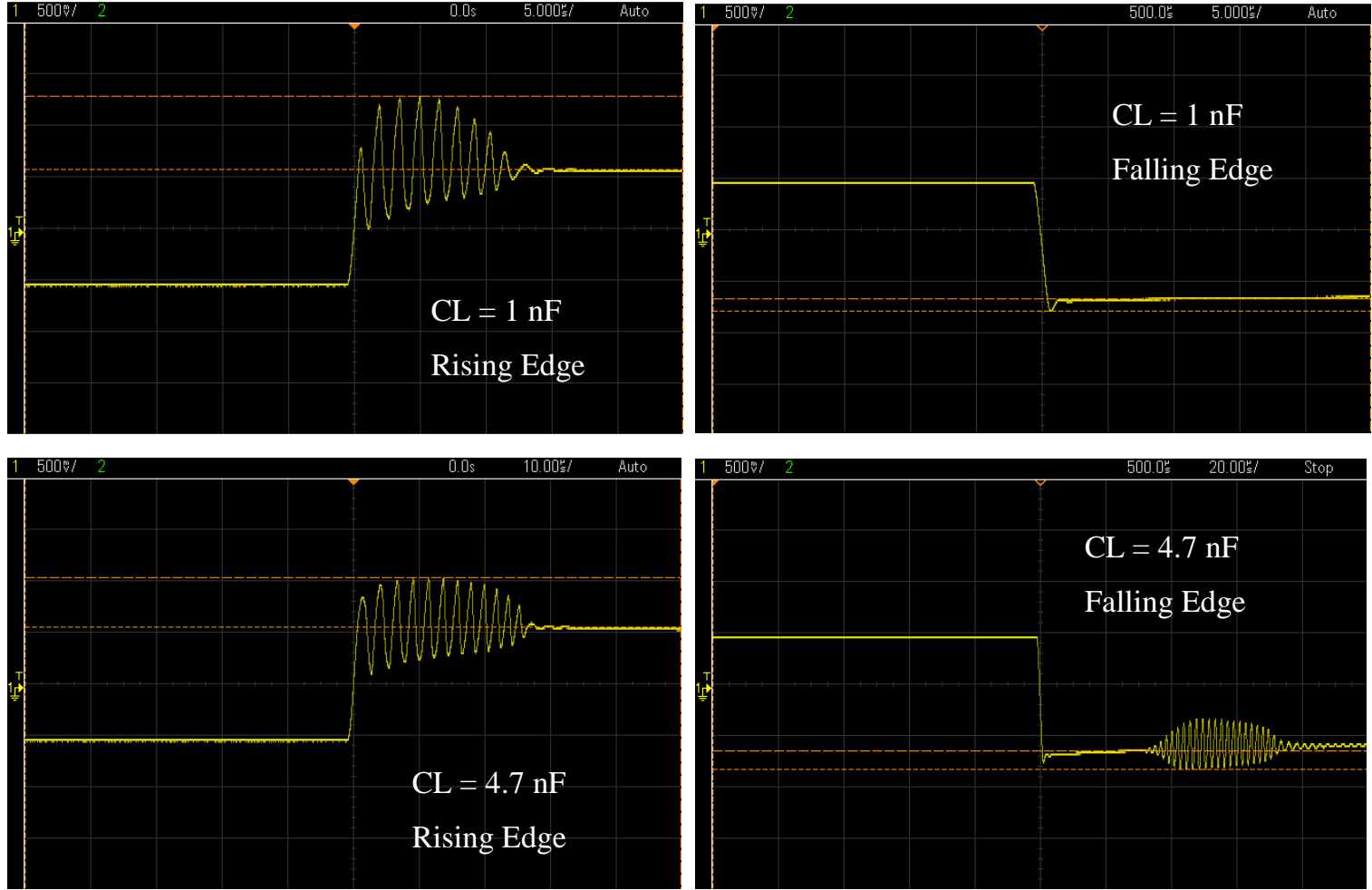

Figure 44. Unfortunately, LMC662 is a bit more stubborn with its natural frequency, a greater damping ratio is required. The falling edge on a $1 \mathrm{nF}$ load is well-behaved, but the rising edge rings for about $15 \mu \mathrm{s}$ most likely due to a nonsymmetrical output impedance.

\section{LMC662 Compensated Linear Signal Response}
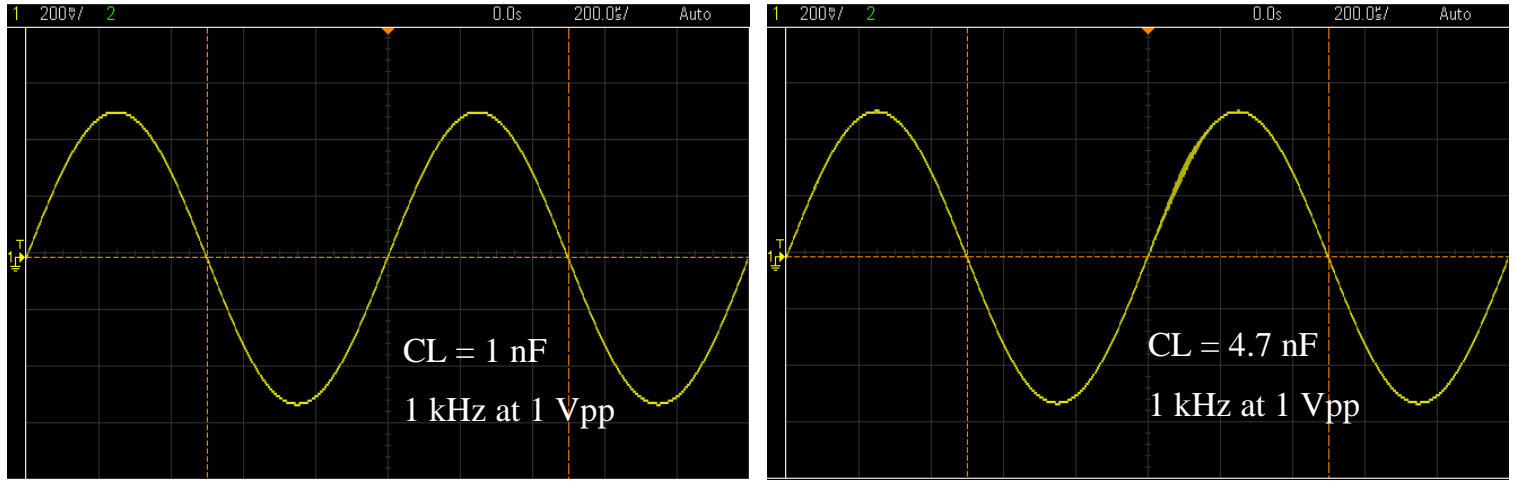

Figure 45. The compensated amplifier is able to drive capacitive loads with a linear signal without any noticeable distortion or ringing artifacts. 


\subsection{LTC6084 Composite Compensation}

Recalling characterization data from $\S 2.3 .3$, values for $C_{C}$ and $R_{C}$ are calculated under the constraints $\zeta=1$ and $\eta_{R}=2 \eta_{C}$.

\begin{tabular}{|c|ccc|cc|}
\hline Load & \multicolumn{3}{|c|}{ Observed Instability } & \multicolumn{2}{c|}{ Compensation Components } \\
\hline CL $(\mathbf{n F})$ & $\mathbf{f , o s c}(\mathbf{k H z})$ & $\mathbf{f , p o l e}(\mathbf{k H z})$ & $\mathbf{r}_{\mathbf{o}}(\mathbf{\Omega})$ & $\mathbf{C c}(\mathbf{n F})$ & $\mathbf{R c}(\mathbf{\Omega})$ \\
\hline 1 & 834 & 331.2 & 481 & 1.7 & 221 \\
\hline 4.7 & 240 & 27.4 & 1235 & 2.4 & 251 \\
\hline 10 & 130 & 8.0 & 1978 & 3.3 & 281 \\
\hline 47 & 70.5 & 2.4 & 1431 & 10.5 & 144 \\
\hline 100 & 58 & 1.6 & 994 & 19.9 & 90 \\
\hline
\end{tabular}

Table 12. Compensation components calculated for a critically damped response.

With precise values calculated, standard components close in value must be selected based on availability. The additional required $R_{1}$ and $C_{1}$ are calculated and selected as well, and the resulting damping and compensation coefficients are determined.

\begin{tabular}{|c|cc|cc|ccc|}
\hline Load & \multicolumn{2}{|c|}{ Compensation Components } & \multicolumn{4}{|c|}{ Additional Components } & \multicolumn{3}{|c|}{ Stability and Coefficients } \\
\hline $\mathbf{C L}(\mathbf{n F})$ & $\mathbf{C c}(\mathbf{n F})$ & $\mathbf{R c}(\boldsymbol{\Omega})$ & $\mathbf{R 1}(\boldsymbol{\Omega})$ & $\mathbf{C 1}(\mathbf{n F})$ & Damping $(\boldsymbol{\zeta})$ & $\boldsymbol{\eta}_{\boldsymbol{R}}$ & $\boldsymbol{\eta}_{\boldsymbol{C}}$ \\
\hline 1 & 1.5 & 200 & $3 \mathrm{k}$ & 22 & 1.13 & 3.4 & 1.7 \\
\hline 4.7 & 2.2 & 240 & $6.8 \mathrm{k}$ & 68 & 1.10 & 6.1 & 3.1 \\
\hline 10 & 3.3 & 270 & $8.2 \mathrm{k}$ & 100 & 1.04 & 8.3 & 4.0 \\
\hline 47 & 10 & 130 & $5.6 \mathrm{k}$ & 470 & 1.15 & 12.0 & 5.7 \\
\hline 100 & 15 & 100 & $4.3 \mathrm{k}$ & 680 & 1.16 & 10.9 & 7.7 \\
\hline
\end{tabular}

Table 13. Resistors and capacitors rounded to standard values so damping ratio stays above 1. 


\section{LTC6084 Compensated Impulse Response}
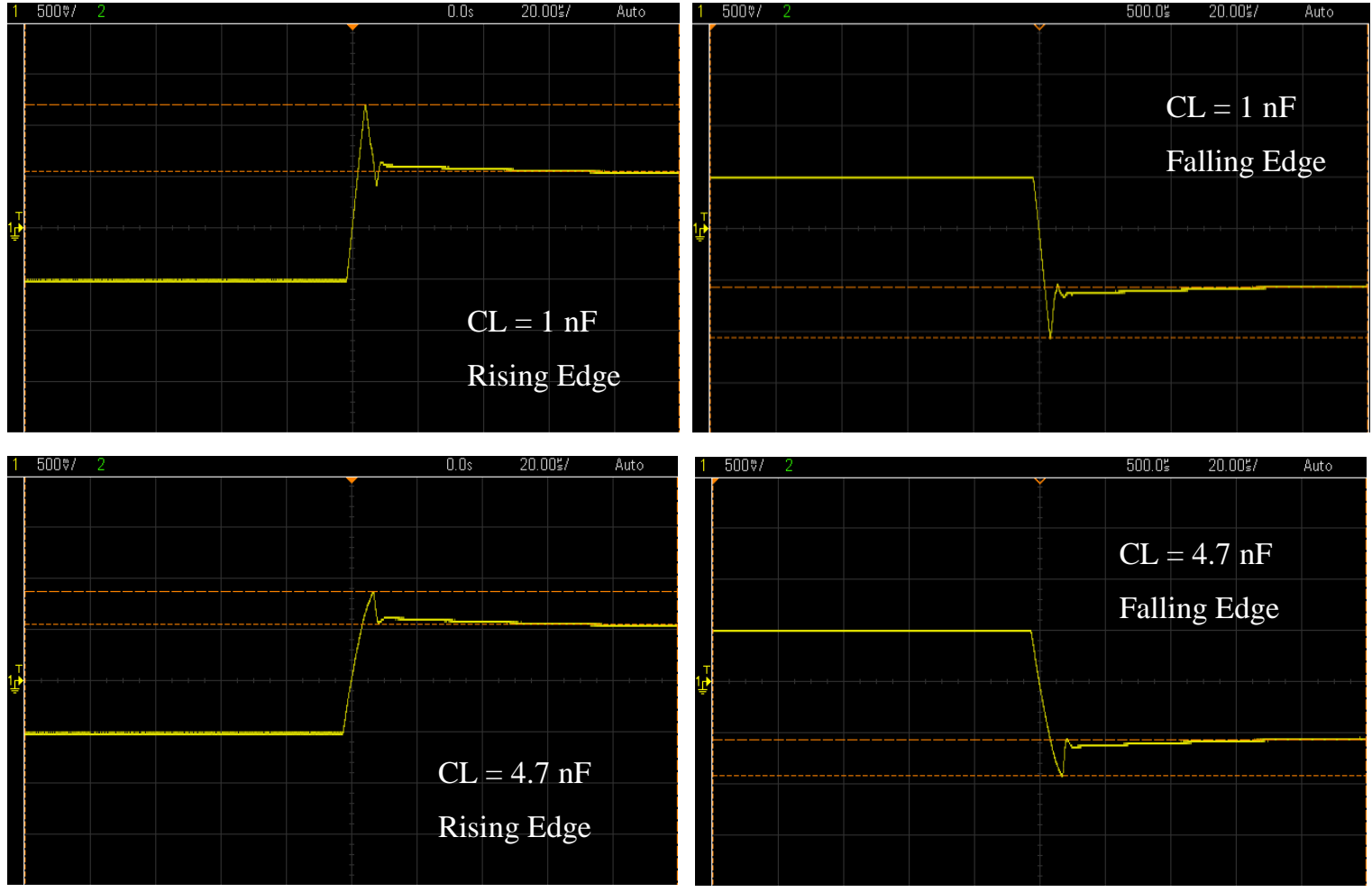

Figure $46.1 \mathrm{nF}$ and $4.7 \mathrm{nF}$ loads have about $50 \%$ and $20 \%$ overshoot, respectively, that die out very quickly within about $5 \mu \mathrm{s}$. Edge transitions are nearly symmetric, which underscores the op-amp's

symmetric and linear design.

\section{LTC6084 Compensated Linear Signal Response}
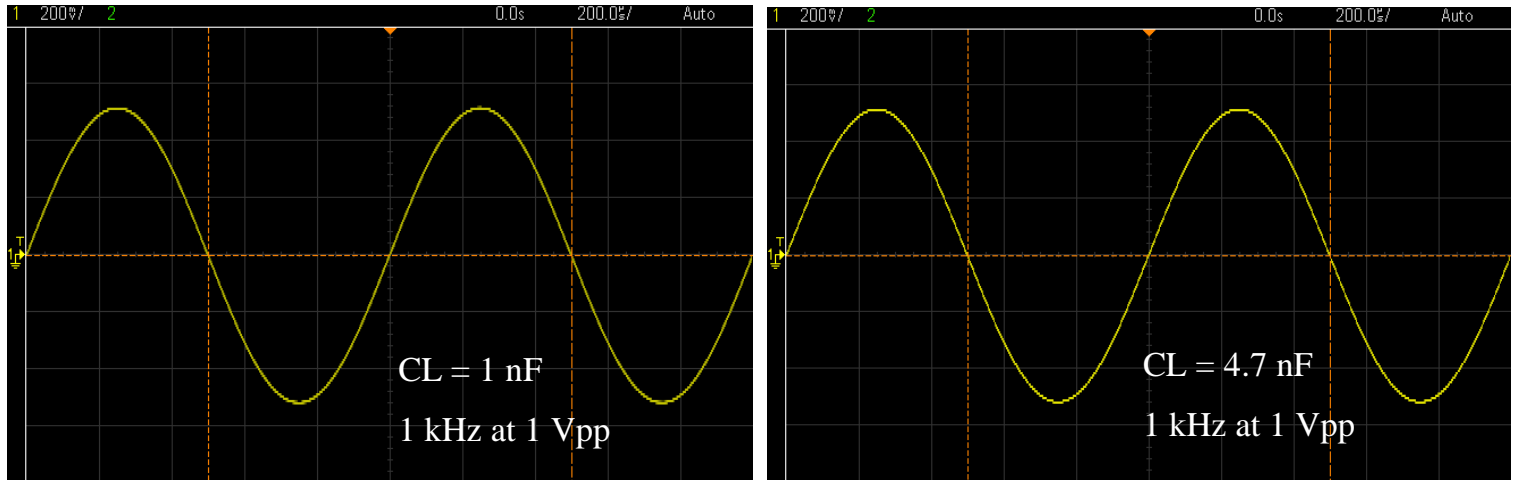

Figure 47. The compensated amplifier is able to drive capacitive loads with a linear signal without any noticeable distortion or ringing artifacts. 


\subsection{Results Discussion}

All three devices used here are general purpose op-amps not designed to handle heavy reactive loads. As a before-and-after comparative measure Figure 48 below recalls the oscilloscope captures from Chapter 2 illustrating unity feedback instability.
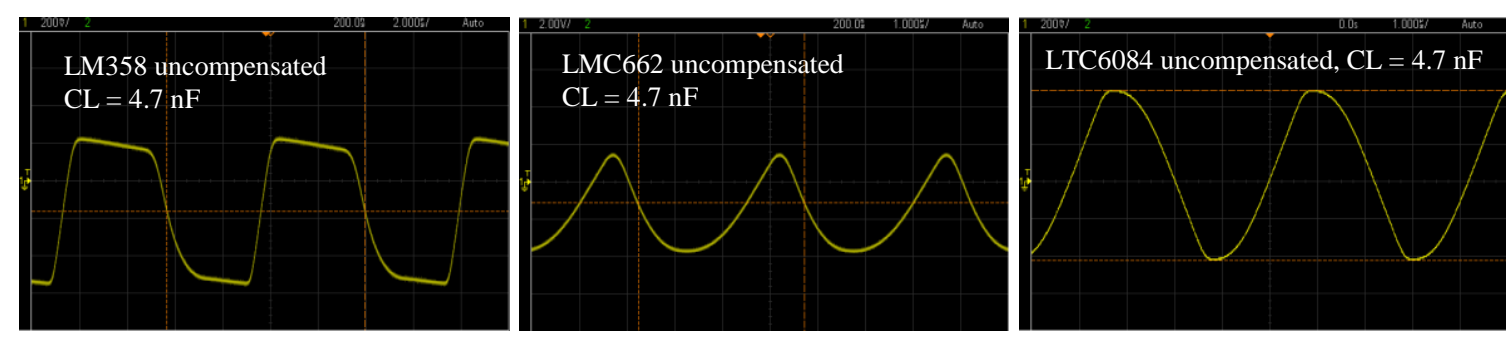

Figure 48. Instability from driving a capacitive load with grounded input (zero-input response).

To properly evaluate the compensation results, the degree and nonlinearity in each unstable output should first be noted. It's not a coincidence that the amount of distortion apparent in signals above inversely correlates with the cost of each device.

LM358 is currently one of the cheapest available op-amps making it an obvious choice in application, but its nonsymmetrical design can make it difficult to stabilize entirely as evidenced by Figure 42. With a targeted damping ratio of 1.07, there is still slight ringing on the falling edge. The likely cause is output impedance not being equal while sinking and sourcing current.

LMC662 could not be completely stabilized with composite compensation. The presence of ringing on the rising edge indicates significantly larger output impedance while sourcing current (Figure 44). The redeeming factor, however, is its compensated ability to drive linear signals (Figure 45) into a capacitive load.

LTC6084 is the most costly of the three, and exhibits the least distorted waveform when unstable, indicating a well-designed architecture. This is apparent in the compensated response where the rising and falling edges have a nearly symmetric overshoot and damping shown in Figure 46.

To generalize observations, a targeted damping of at least 0.5 stabilized the unforced response (i.e. grounded input), but $\zeta>1$ was required to stabilize any non-zero input signal. 


\section{Chapter 7: Conclusion}

This thesis has presented a design basis for use in frequency compensating opamps when the load is heavily capacitive. An analytical approach was taken to investigate stability issues resulting from this type of load. It was then shown that a well-designed passive network can be used to regain stability. The technique developed here allows for a more versatile use of lower performance devices. The designer only needs to know minimal device parameters and how much power and voltage swing can be traded off for the desired scale of compensation.

While it is true that specialized, more expensive op-amps can be used to buffer capacitive loads the composite compensation method can allow it to be done at a much lower cost. The devices compensated in Chapter 6 showed appreciable performance with the natural frequency being absent. The composite compensation strategy finds excellent application in any circuit where a load's capacitive nature destabilizes feedback.

\subsection{Topics for Further Study}

The composite compensation method is successfully demonstrated on a very small sample of three op-amps among thousands of available devices. It may be of use to test compensation effectiveness under a more specific set of parameters such as architecture, load rating, process technology, nominal GBW, etc. Even verifying Stability testing of op-amps designed for broader bandwidths (on the scale of 10's of MHz) can also be done because they are rated for even smaller capacitive loads.

Simulations predicted more current and larger voltage swing required at higher frequencies, but this could not be verified in the lab. Limited slew rate distorted signals well before such high frequencies could be reached so research into an experiment that verifies this tradeoff can be done. Furthermore, to supplement the background and theory behind composite compensation, a Root Locus simulation can be performed in MATLab to demonstrate loop poles becoming complex as the load becomes more capacitive or gain grows larger. 


\section{REFERENCES}

[1] A. Sedra and K. Smith, "Differential and Multistage Amplifiers", in MicroelectronicCircuits, 6th ed. Oxford. Oxford University Press, 2009, ch 8.

[2] Microchip Tech, K. Blake, Appl Note AN884, Driving capacitive loads with op amps, 2003.

[3] W. Brogan, Modern Control Theory, $3^{\text {rd }}$ ed, Prentice Hall, 1990, pp. 39-63.

[4] Maxim Integrated, M. Fortunato, Simulation shows how real op amps can drive capacitive loads, 2013.

[5] S. Franco, Analog Integrated Circuits: Discrete and Integrated, $1^{\text {st }} \mathrm{ed}$, McGraw-Hill, New York, 2015, ch 5, 7.

[6] S. Franco, Design with Operational Amplifiers and Analog Integrated Circuits, $4^{\text {th }}$ ed, McGraw-Hill, New York, 2014, ch 5, 6, 8.

[7] R. Jaeger and T. Blalock, Microelectronic Circuit Design, $4^{\text {th }}$ ed. McGraw-Hill, New York, 2011, ch 10, 11, 12, 17.

[8] ST Microelectronics, Operational amplifier stability compensation methods for capacitive loading applied to TS507, 2007.

[9] T. M. Waddel, “A Design Basis for Composite Cascode Stages”, M.S. thesis, Dept. of Electrical and Computer Engr., BYU, Provo, UT, 2012. 


\section{APPENDICES}

\section{A1 Deriving the Idealized Op-Amp Model}

Shown below is the generally accepted model for an op-amp with DC gain $A_{d c}$, dominant pole $\omega_{0}$, and secondary pole $\omega_{1}$. Typically, the DC gain is very high $(>100$ $\mathrm{dB}$ ), the dominant pole hovers between 1 and $10 \mathrm{~Hz}$, while the secondary pole is placed at least twice the unity-gain frequency (at rated loading). Negative feedback is applied with $\beta=1$.

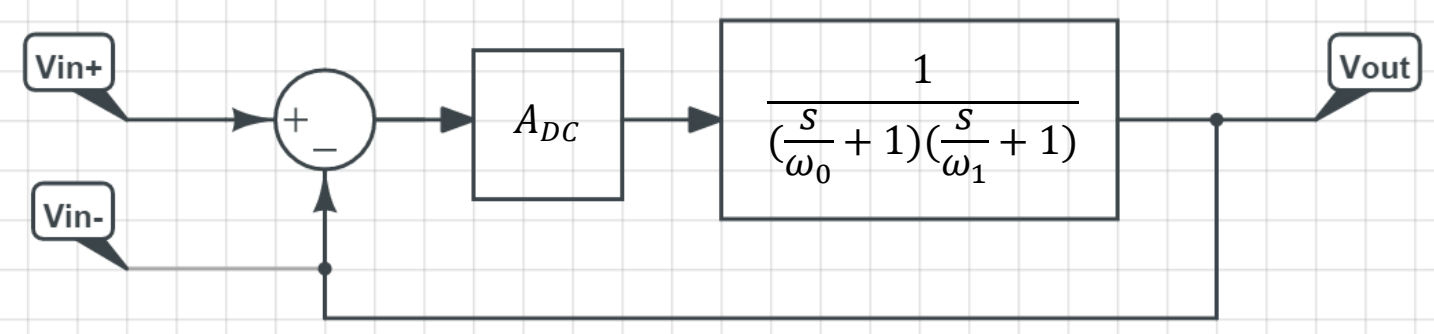

Figure 49. Standard two-pole model of an op-amp with gain DC gain block and unity feedback.

The transfer characteristic between Vout and Vin+ can be shown.

$$
H(s)=\frac{A_{d c} \frac{1}{\left(\frac{s}{\omega_{0}}+1\right)\left(\frac{s}{\omega_{1}}+1\right)}}{1+A_{d c} \frac{1}{\left(\frac{s}{\omega_{0}}+1\right)\left(\frac{s}{\omega_{1}}+1\right)}}=\frac{A_{d c}}{\left(\frac{s}{\omega_{0}}+1\right)\left(\frac{s}{\omega_{1}}+1\right)+A_{d c}}
$$

The characteristic equation of this feedback control system is extracted and rearranged.

$$
\begin{gathered}
\left(\frac{s}{\omega_{0}}+1\right)\left(\frac{s}{\omega_{1}}+1\right)+A_{d c}=0 \\
\Rightarrow s^{2}+s\left(\omega_{0}+\omega_{1}\right)+\left(A_{d c}+1\right) \omega_{0} \omega_{1}=0
\end{gathered}
$$

Two approximations can be made: $\omega_{0}+\omega_{1} \approx \omega_{1}$ because $\omega_{1} \gg \omega_{0}$ and $\left(A_{d c}+1\right) \approx$ $A_{d c}$. Furthermore, $A_{d c} * \omega_{0}=\omega_{u}$ is the known GBW of the device, so the characteristic equation can be simplified to the following.

$$
s^{2}+s \omega_{1}+\omega_{u} \omega_{1}=0
$$

In Chapters 1 and 3 , it was discussed that the secondary pole gets pulled inward and is defined by $\omega_{1}=1 / r_{0} C_{L}$. Substituting this in, the characteristic equation becomes identical to that which was derived using the idealized model in Chapter 3.

$$
s^{2}+\frac{s}{r_{o} C_{L}}+\frac{\omega_{u}}{r_{o} C_{L}}=0
$$


This implies both systems have the same poles, damping ratio, and natural frequency, and are thus identical as well from a stability standpoint.

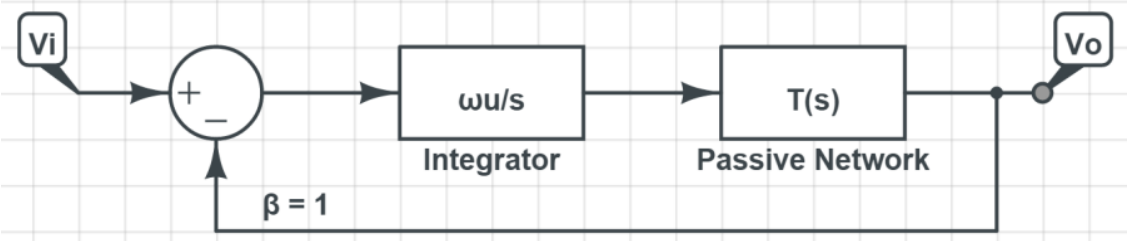

Figure 50. Two-pole model can be simplifed to an idealized type-I second order model.

For $T(s)=\frac{1}{r_{o} C_{L} s+1}$, Figure 50 has the same characteristic equation defined by Equation A.4. 